\title{
Position-space cuts for Wilson line correlators
}

\author{
Eric Laenen, ${ }^{a, b, c}$ Kasper J. Larsen ${ }^{a, d}$ and Robbert Rietkerk ${ }^{a, b}$ \\ ${ }^{a}$ Nikhef, Theory Group, \\ Science Park 105, 1098 XG Amsterdam, The Netherlands \\ ${ }^{b}$ ITFA, University of Amsterdam, \\ Science Park 904, 1018 XE Amsterdam, The Netherlands \\ ${ }^{c}$ ITF, Utrecht University, \\ Leuvenlaan 4, 3584 CE Utrecht, The Netherlands \\ ${ }^{d}$ Institute for Theoretical Physics, ETH Zürich, \\ 8093 Zürich, Switzerland \\ E-mail: Eric.Laenen@nikhef.nl, Kasper.Larsen@phys.ethz.ch, \\ Robbert.Rietkerk@nikhef .nl
}

ABSTRACT: We further develop the formalism for taking position-space cuts of eikonal diagrams introduced in ref. [1]. These cuts are applied directly to the position-space representation of any such diagram and compute its discontinuity to the leading order in the dimensional regulator. We provide algorithms for computing the position-space cuts and apply them to several two- and three-loop eikonal diagrams, finding agreement with results previously obtained in the literature. We discuss a non-trivial interplay between the cutting prescription and non-Abelian exponentiation. We furthermore discuss the relation of the imaginary part of the cusp anomalous dimension to the static interquark potential.

Keywords: Wilson, 't Hooft and Polyakov loops, Scattering Amplitudes, QCD, Resummation

ARXIV EPRINT: 1505.02555 


\section{Contents}

1 Introduction 1

2 Imaginary parts of eikonal diagrams and their physical origin 4

3 Position-space cuts of eikonal diagrams without internal vertices 11

4 Examples $\quad \mathbf{1 5}$

$\begin{array}{lll}\text { 4.1 The non-planar two-loop ladder diagram } & 15\end{array}$

4.2 Three-loop non-planar ladder diagram 20

4.3 Two-loop web with three Wilson lines 24

5 Position-space cuts of eikonal diagrams with internal vertices 30

6 Conclusions $\quad 36$

$\begin{array}{ll}\text { A Real and imaginary parts of multiple polylogarithms } & 37\end{array}$

B Algorithm for achieving canonical-form polylogarithms 44

\section{Introduction}

The infrared singularities of gauge theory scattering amplitudes play a fundamental role in particle physics for phenomenological as well as more theoretical studies. Determining the long-distance singularities is necessary for combining the real and virtual contributions to the cross section, as the divergences of the separate contributions only cancel once they are added. Infrared singularities moreover dictate the structure of large logarithmic contributions to the cross section, allowing such terms to be resummed - which is in many cases required in order to obtain reliable perturbative predictions. Beyond their significance to collider phenomenology, long-distance singularities are highly interesting from a theoretical point of view. Among several properties, they have a universal structure among different gauge theories; moreover, their exponentiation properties [2-14] and their relation to the renormalization of Wilson line correlators [15-21] allow their perturbative expansion to be explored to all orders, a feat currently unattainable for complete scattering amplitudes.

The basic tool for computing the infrared singularities of any scattering amplitude is provided by the eikonal approximation. In this limit the momenta of the soft gauge bosons emitted between the partons emerging from the hard interaction are neglected with respect 
to the hard momenta $p_{i}$. As a result, each hard parton $i$ simply acts as a source of soft gluon radiation and is accordingly replaced by a semi-infinite Wilson line

$$
\Phi_{v_{i}} \equiv \mathcal{P} \exp \left(i g \int_{0}^{\infty} \mathrm{d} t v_{i} \cdot A\left(t v_{i}\right)\right),
$$

which extends from time $t=0$, when the hard scattering takes place, to infinity along the classical trajectory of the hard parton, traced out by its four-velocity $v_{i}^{\mu}$. The long-distance singularities of the scattering amplitude of the hard partons are then encoded in the eikonal amplitude

$$
\mathcal{S}\left(\gamma_{i j}, \epsilon\right) \equiv\left\langle 0\left|\Phi_{v_{1}} \otimes \Phi_{v_{2}} \otimes \cdots \otimes \Phi_{v_{n}}\right| 0\right\rangle,
$$

which has the same soft singularities as the original amplitude, but is much simpler to compute. An important feature of the eikonal amplitude (1.2) is the fact that it depends on the kinematics only through the angles $\gamma_{i j}$ between the four-velocities (defined through $\left.\cosh \gamma_{i j} \equiv\left|v_{i} \cdot v_{j}\right|\right)$. Before renormalization, the integrals involved in the loop-level contributions to $\mathcal{S}$ are thus scale invariant and vanish identically. This in turn allows the infrared singularities at any loop order to be computed by studying the ultraviolet renormalization factor of the Wilson line correlator (1.2) [19, 21-25]. This renormalization factor forms a matrix in the space of color configurations available for the scattering process at hand, referred to as the soft anomalous dimension matrix. In processes involving only two Wilson lines, this matrix reduces to the cusp anomalous dimension, a quantity which has been computed in QCD up to three loops [21, 26, 27]. In $\mathcal{N}=4$ super Yang-Mills theory, the cusp anomalous dimension is known to three loops [28], and partial results have been obtained at four loops $[29,30]$. For multi-parton amplitudes, the soft anomalous dimension matrix has been computed through two loops for massless [31, 32] as well as massive [33-37] Wilson lines. Recently, much progress has been made toward the calculation of the soft anomalous dimension matrix at three loops [38, 39].

In this paper we continue exploring a notion of cuts of eikonal diagrams (i.e., the diagrams contributing to the eikonal amplitude) introduced in ref. [1]. Applied to any eikonal diagram, the cuts compute the discontinuities of the diagram, in analogy with the Cutkosky rules for standard Feynman diagrams. The discontinuities are in turn readily combined to produce the imaginary part of the diagram, a direct computational method of which is desirable in several contexts. Indeed, collinear factorization theorems for non-inclusive observables were pointed out in refs. $[40,41]$ to be violated due to exchanges of Glauberregion (i.e., maximally transverse) gluons. The resulting factorization-breaking terms are purely imaginary and take the form of the non-Abelian analog of the QED Coulomb phase. Therefore, by utilizing the all-order exponentiation property of the eikonal amplitude, the latter could be obtained directly by computing the imaginary part of the exponent. The resulting non-Abelian Coulomb phase [42, 43] may also aid studies of interference effects. The importance of understanding the imaginary part of eikonal diagrams has also recently been highlighted in studies regarding rapidity gaps [44, 45]. Moreover, cuts of Wilson line correlators are naturally relevant for cross section calculations [46, 47].

A cutting prescription for eikonal diagrams may also provide the first step toward extending the modern unitarity method [48-57] to eikonal amplitudes. The development 
of the unitarity method has led to a dramatic improvement in the ability to compute loop-level (non-eikonal) scattering amplitudes at high multiplicity. In this approach, the loop amplitude is decomposed into a linear basis of loop integrals which are computed independently (for example, by means of Feynman parametrization, or differential equations $[58,59])$. The calculation of the loop amplitude is then reduced to the problem of determining the integral coefficients. This step is performed by applying to both sides of the basis decomposition of the loop amplitude a number of cuts which have the effect of putting the internal lines on shell. In basic unitarity (as opposed to generalized unitarity), the cuts employed measure the discontinuity of the amplitude in its various kinematical channels. Unitarity has proven highly successful, notably in computing one-loop amplitudes with many partons in the final state. It is therefore natural to look for extensions of this method to other physical quantities with a perturbative expansion.

It should be emphasized that Cutkosky rules for eikonal diagrams have been introduced previously in the literature, as a cut prescription applied directly to the momentum-space representation of the diagrams [21]. In contrast, the cuts introduced in ref. [1] and further studied here are applied to the position-space representation of the eikonal diagrams. A notion of position-space cuts of non-eikonal diagrams exists in the literature in the form of a cutting equation that follows from Veltman's largest-time equation [60]. However, that notion is conceptually different from the position-space cuts in this paper, since the former has the effect of cutting a given diagram in two disconnected subdiagrams while the latter does not. Moreover, in practice, the largest-time equation is typically not applied directly, but rather serves to derive the momentum-space Cutkosky rules, which in turn are used to obtain the imaginary part of a diagram. As already observed in ref. [1], position-space cuts provide a substantial simplification over momentum-space cuts in the computation of imaginary parts of eikonal diagrams. There has been recent interest in the literature in studying Wilson line correlators in position space, in particular refs. [61-63] which investigate the structure of infrared singularities and factorization in position space. Moreover, position-space analogs of generalized unitarity cuts of Wilson line correlators were recently introduced in ref. [64].

The structure of this paper is as follows. In section 2 we discuss the origin of the imaginary part of Wilson line correlators from the point of view of causality as well as unitarity. We then show how the imaginary part can be computed from the position- and the momentum-space representations at one loop. In section 3 we review the formula in ref. [1] for the imaginary part of $L$-loop eikonal diagrams containing no internal (i.e., threeor four-gluon) vertices to the leading order in the dimensional regulator $\epsilon$. We furthermore discuss the relation of the discontinuities of the diagrams to their imaginary part. In section 4 we apply the formalism to compute the imaginary part of a number of two- and three-loop diagrams and discuss a non-trivial interplay between the cutting prescription and non-Abelian exponentiation. In section 5 we turn to formulas for the imaginary part of eikonal diagrams with internal vertices and provide details on its computation. We give our conclusions in section 6. Appendix A explains our method for computing the principalvalue integrals involved in the cutting prescription. In appendix B we present our algorithm for re-expressing multiple polylogarithms in terms of ones with constant indices. 


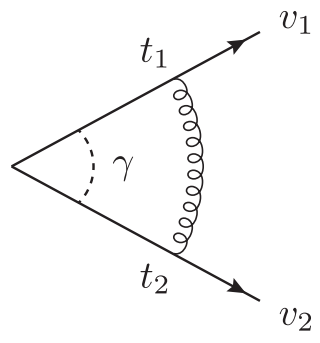

(a): $v_{1} \cdot v_{2}>0$

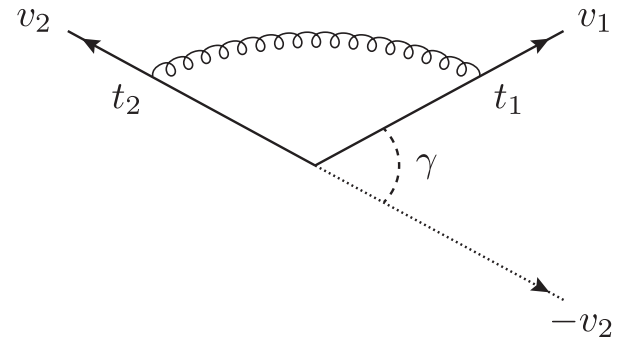

(b): $v_{1} \cdot v_{2}<0$

Figure 1. One-loop eikonal diagrams. In (a) both Wilson lines represent final-state partons (as for example in $e^{+} e^{-} \rightarrow q \bar{q}$ ). Thus, the external velocities are in the region $v_{1} \cdot v_{2}>0$, and the partons can become lightlike separated. In (b) one Wilson line represents a final-state parton, and one represents an initial-state parton (as for example in deep inelastic scattering). Thus, the external velocities are in the region $v_{1} \cdot v_{2}<0$, and the partons are never lightlike separated.

\section{Imaginary parts of eikonal diagrams and their physical origin}

In this section we will discuss the origin of the imaginary part of Wilson line correlators from the point of view of causality as well as unitarity. These viewpoints are naturally provided by the representation of the correlator in position and momentum space, respectively. We will show how the imaginary part can be computed directly from each of the respective integral representations at one loop.

We adopt the convention that all velocities are outgoing, such that the velocities associated with outgoing and incoming states respectively have positive and negative time components. We will take the gauge group to be $\mathrm{SU}(N)$ and work in Feynman-'t Hooft gauge with $(+,-,-,-)$ spacetime signature. Ultraviolet divergences will be regulated by computing all diagrams in $D=4-2 \epsilon$ dimensions with $\epsilon>0$. To avoid complications arising from regulating collinear singularities, we take all velocities to be time-like, $v_{i}^{2}=1$.

We start our investigations by examining the simplest eikonal diagram, the one-loop exchange illustrated in figure 1. In both kinematic regions (a) and (b), the positionspace representation of the diagram is straightforwardly obtained by direct perturbative expansion ${ }^{1}$ in $g$ of the correlator (1.2) and takes the form

$$
F^{(1)}=C^{(1)} \mu^{2 \epsilon} \int_{0}^{\infty} \mathrm{d} t_{1} \int_{0}^{\infty} \mathrm{d} t_{2} \frac{v_{1} \cdot v_{2}}{\left[-\left(t_{1} v_{1}-t_{2} v_{2}\right)^{2}+i \eta\right]^{1-\epsilon}},
$$

where the prefactor is defined as $C^{(1)} \equiv g^{2} C_{F} \frac{\Gamma(D / 2-1)}{4 \pi^{D / 2}}$, with $C_{F}=\frac{N^{2}-1}{2 N}$ denoting the quadratic Casimir of the fundamental representation. Furthermore, $t_{1}, t_{2}$ have the dimension of time and denote the positions of the attachment points of the soft-gluon propagator on the Wilson lines spanned by the four-velocities $v_{1}$ and $v_{2}$.

The integrations in eq. (2.1) produce an infrared divergence which can be extracted via the change of variables $\left(t_{1}, t_{2}\right)=(\lambda x, \lambda(1-x))$ with $0 \leq x \leq 1$, where $\lambda$ has the dimension

\footnotetext{
${ }^{1}$ See chapter 8 of ref. [65] for the position-space Feynman rules.
} 
of length,

$$
F^{(1)}=C^{(1)} \mu^{2 \epsilon} \int_{0}^{\infty} \frac{\mathrm{d} \lambda}{\lambda^{1-2 \epsilon}} \int_{0}^{1} \mathrm{~d} x \frac{v_{1} \cdot v_{2}}{\left[-\left(x v_{1}-(1-x) v_{2}\right)^{2}+i \eta\right]^{1-\epsilon}} .
$$

Indeed, the $\lambda$-integral is has an infrared divergence, owing to the exchange of gluons of increasingly longer wavelength as $\lambda \rightarrow \infty$. This divergence can be regularized in a gauge invariant fashion by introducing an exponential damping factor $e^{-\Lambda \lambda}$ with $\Lambda \ll 1$, whereby it becomes

$$
\mu^{2 \epsilon} \int_{0}^{\infty} \frac{\mathrm{d} \lambda e^{-\Lambda \lambda}}{\lambda^{1-2 \epsilon}}=\Gamma(2 \epsilon)\left(\frac{\mu}{\Lambda}\right)^{2 \epsilon}=\frac{1}{2 \epsilon}\left(\frac{\mu}{\Lambda}\right)^{2 \epsilon}+\mathcal{O}\left(\epsilon^{0}\right) .
$$

The two diagrams in figure 1 have the same integrand; however, as the external kinematics is taken from the distinct regions $v_{1} \cdot v_{2}>0$ and $v_{1} \cdot v_{2}<0$, the integrations will produce distinct results. It is most convenient to compute the diagram in figure 1(b) first and obtain the result for figure 1(a) by analytic continuation as follows. For the diagram in figure 1(b), we may define the deflection angle $\gamma>0$ such that $\cosh \gamma=-v_{1} \cdot v_{2}$, in terms of which the diagram in figure 1(b) becomes, to the leading order in $\epsilon$,

$$
F_{1(\mathrm{~b})}^{(1)}=\frac{C^{(1)}}{2 \epsilon}\left(\frac{\mu}{\Lambda}\right)^{2 \epsilon} \gamma \operatorname{coth} \gamma
$$

Likewise, for the diagram in figure 1(a), we may define the cusp angle $\gamma>0$ such that $\cosh \gamma=v_{1} \cdot v_{2}$. The integrated expression for this diagram can thus be obtained from eq. (2.4) by replacing $\gamma^{(\mathrm{b})} \rightarrow \pi i-\gamma^{(\mathrm{b})}=\gamma^{(\mathrm{a})}$,

$$
F_{1(\mathrm{a})}^{(1)}=\frac{C^{(1)}}{2 \epsilon}\left(\frac{\mu}{\Lambda}\right)^{2 \epsilon}(\gamma-\pi i) \operatorname{coth} \gamma .
$$

We observe that the imaginary parts of the eikonal diagrams in figures $1(\mathrm{a})$ and $1(\mathrm{~b})$ are respectively non-vanishing and vanishing. Before turning to the question of how the imaginary parts of the diagrams in eqs. (2.4)-(2.5) may be extracted from their integral representation in eq. (2.2), let us consider their physical origin and interpretation.

From the position-space representation (2.1) of the eikonal diagram, the origin of the imaginary part may be understood from a simple causality consideration as follows. As our focus is on computing the imaginary part to the leading order in $\epsilon$, the $\epsilon$ in the propagator exponent may be dropped once the infrared divergence has been extracted. After moreover stripping off real prefactors from eq. (2.1), the integral takes the form,

$$
\int_{0}^{\infty} \mathrm{d} t_{1} \int_{0}^{\infty} \mathrm{d} t_{2} \frac{1}{\left(t_{1} v_{1}-t_{2} v_{2}\right)^{2}-i \eta}
$$

Now, for the kinematics corresponding to the diagram in figure 1(a), there are regions $\frac{t_{1}}{t_{2}}=e^{ \pm \gamma}$ within the integration domain where $\left(t_{1} v_{1}-t_{2} v_{2}\right)^{2}=0$. Here the $-i \eta$ term becomes relevant and generates an imaginary part. What is happening physically at such times $t_{1}, t_{2}$ is that the two partons traveling along $v_{1}$ and $v_{2}$ become lightlike separated. This is illustrated in figure 2(a). As a result, the phases of their states will change through 


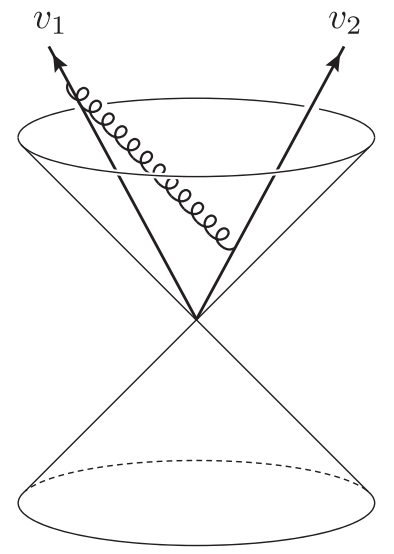

(a)

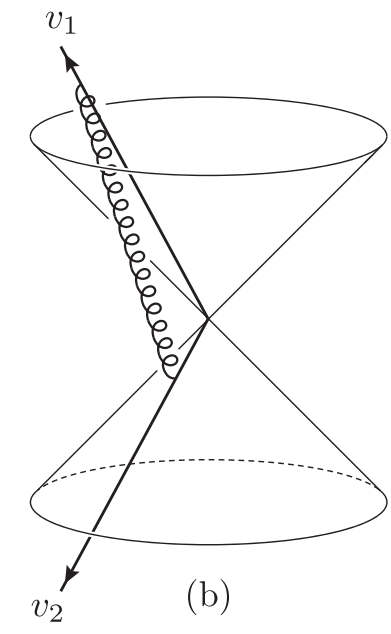

(b)

Figure 2. The one-loop eikonal diagrams of figure 1 embedded in a space-time diagram. In (a) both Wilson lines are confined to the interior of the future light cone, describing two final state partons. In this case the partons can become lightlike separated, which is illustrated by the exchange of a lightlike gluon (i.e. a gluon that is aligned to the light cone). In (b) there is one incoming parton inside the past light cone and an outgoing parton inside the future light cone. These partons are never lightlike separated. Indeed, the gluon stretching between these two Wilson lines is necessarily time-like (i.e. off shell).

exchanges of lightlike gluons (or photons) - leading to observable consequences that will be discussed shortly. In contrast, for external kinematics corresponding to the diagram in figure 1(b), the integral in eq. (2.6) has a vanishing imaginary part: the denominator $\left(t_{1} v_{1}-t_{2} v_{2}\right)^{2}$ is strictly positive within the region of integration, and the $-i \eta$ can therefore be dropped. In this situation, the partons are never lightlike separated, as illustrated in figure 2(b), and the phases of their states cannot change through exchanges of lightlike massless gauge bosons.

These observations on the evolution of the phases of the hard-parton states suggest that the imaginary part of the correlator of two Wilson lines defines an interparton potential. Indeed, in the non-relativistic limit, the final and initial two-particle states are related in the interaction picture through time evolution by $|f\rangle_{I}=e^{i \int_{0}^{\infty} \mathrm{d} t e^{-\Lambda t} V_{I} t}|i\rangle_{I}$ where $V_{I}$ denotes the interaction potential. The relation of the correlator to a non-relativistic potential can be made precise in the situation where the pair of energetic particles carry no color charges, as for example in the case of an $e^{+} e^{-}$pair. In Abelian gauge theories, the correlator of two Wilson lines can be written as the exponential of the sum of connected diagrams [2],

$$
W \equiv\left\langle\Phi_{v_{1}} \Phi_{v_{2}}\right\rangle=\exp \left(F^{(1)}+\mathcal{O}\left(g^{4}\right)\right)
$$

where $F^{(1)}$ is the one-loop diagram in figure 1, and the additional diagrams contain a single lepton loop connected to the Wilson lines by an arbitrary (even) number of soft-photon exchanges. Using the result for the diagram $F^{(1)}$ computed for time-like kinematics in eq. (2.5) (with $C_{F} \rightarrow 1$ to recover the Abelian case), the anomalous dimension of the 
Wilson-line correlator — i.e., the cusp anomalous dimension — evaluates to

$$
\Gamma_{\text {cusp }}(\gamma) \equiv-\lim _{\epsilon \rightarrow 0} \frac{\mathrm{d} \log W}{\mathrm{~d} \log \mu}=-\frac{g^{2}}{4 \pi^{2}}(\gamma-\pi i) \operatorname{coth} \gamma .
$$

The non-relativistic limit corresponds to the small-angle regime $\gamma \approx 0$ where the two velocities $v_{1}$ and $v_{2}$ are nearly collinear, and the relative velocity of the hard leptons thus small. Accordingly, expanding eq. (2.8) around $\gamma=0$ and taking the imaginary part, we find

$$
\operatorname{Im} \Gamma_{\text {cusp }}(\gamma)=\frac{g^{2}}{4 \pi \gamma}+\mathcal{O}\left(\gamma^{0}\right) \text {. }
$$

We observe that the imaginary part of the cusp anomalous dimension evaluated in timelike kinematics takes the form of the non-relativistic Coulomb potential (the appropriate dimension of energy is acquired after replacing the angle $\gamma$ by the distance between the two fermions).

This relation does not extend to generic non-Abelian gauge theories, as we will discuss shortly. It does, however, extend to the case of conformal field theories, such as $\mathcal{N}=4$ super Yang-Mills theory, where the state-operator correspondence relates Wilson-line operators in Minkowski space to states in $\mathbb{R} \times \mathrm{AdS}_{3}$. In radial quantization, a pair of Wilson lines intersecting at a cusp angle $\gamma$ with the resulting anomalous dimension $\Gamma_{\text {cusp }}(\gamma)$ is mapped to a pair of static charges in $\mathrm{AdS}_{3}$ separated by a distance of $\gamma$ with an electrostatic energy ${ }^{2}$ of $\operatorname{Im} \Gamma_{\text {cusp }}$ [66]. For small values of the cusp angle, the charges on $\mathrm{AdS}_{3}$ become closer than the curvature scale, and the electrostatic energy takes the form of the non-relativistic interquark potential in flat space $[28,67]$. (The non-relativistic approximation becomes relevant here, as in the small-angle regime $\gamma \approx 0$, the relative velocity of the hard partons is small, as discussed above.)

However, for non-Abelian and non-conformal gauge theories such as QCD, diagrams containing loop corrections to the soft propagators will have a dependence on the beta function, thereby explicitly breaking the scale invariance of the diagram. As a result, in QCD, the imaginary part of the three-loop cusp anomalous dimension $\Gamma_{\text {cusp }}^{(3)}$ differs from the static interquark potential by terms proportional to the beta function [27]. (This can be seen by comparing the $N_{f}^{2}$ contribution to $\Gamma_{\text {cusp }}^{(3)}$, given in eq. (A.2) of ref. [68], against the $N_{f}^{2}$ term of the three-loop static QCD potential, ${ }^{3}$ given in eq. (10) of ref. [69].)

Let us now turn to the question of how the imaginary part of the eikonal diagrams in figure 1 may be obtained from their integral representation in eq. (2.2) where the infrared divergence has been extracted. We will restrict attention to the leading order in the dimensional regulator $\epsilon$, and accordingly drop the $\epsilon$ in the propagator exponent. We can then utilize the formula

$$
\int_{a}^{b} \mathrm{~d} x \frac{f(x)}{D(x) \pm i \eta}=\mathrm{PV} \int_{a}^{b} \mathrm{~d} x \frac{f(x)}{D(x)} \mp \pi i \int_{a}^{b} \mathrm{~d} x f(x) \delta(D(x)),
$$

\footnotetext{
${ }^{2}$ The real part of the cusp anomalous dimension gives rise to an imaginary part of the electrostatic energy. As argued in ref. [66], the resulting non-unitary time evolution is accounted for by the real radiation of soft and collinear gluons along the Wilson lines.

${ }^{3}$ Note that in the literature on the interquark potential, the loop order is often defined as one less than the standard notion.
} 
where PV indicates that the Cauchy principal value prescription is to be applied, and the integration bounds $a$ and $b$ are real numbers. The denominator $D(x)$ is a real-valued polynomial in $x$, and the numerator $f(x)$ is an arbitrary real-valued function with no poles or branch points inside the integration path. As both integrals on the right-hand side of eq. (2.10) are real, this formula achieves a decomposition into a purely real and purely imaginary part.

Accordingly, at one loop, we define the position-space cut prescription

$$
\frac{1}{D(x) \pm i \eta} \stackrel{\text { cut }}{\longrightarrow} \mp \pi i \delta(D(x))
$$

in terms of which it is straightforward to obtain the imaginary part of the diagrams in figure 1 to the leading order in $\epsilon$. For example, considering the time-like kinematics situation in figure 1(a) and applying the prescription (2.11) to eq. (2.2) with the $\epsilon$ in the propagator exponent set to zero, we find

$$
\operatorname{Im} F_{1(\mathrm{a})}^{(1)}=-\pi C^{(1)}\left(v_{1} \cdot v_{2}\right) \mu^{2 \epsilon} \int_{0}^{\infty} \frac{\mathrm{d} \lambda e^{-\Lambda \lambda}}{\lambda^{1-2 \epsilon}} \int_{0}^{1} \mathrm{~d} x \delta\left(\left(x v_{1}-(1-x) v_{2}\right)^{2}\right) .
$$

We can integrate out the delta function by use of the identity

$$
\int_{0}^{1} \mathrm{~d} x \delta\left(A x^{2}+B x+C\right)=\frac{1}{\sqrt{\Delta}} \sum_{i=1,2} \theta\left(\rho_{i}\right) \theta\left(1-\rho_{i}\right),
$$

where $\Delta \equiv B^{2}-4 A C$ and $\rho_{i}$ respectively denote the discriminant and roots of the polynomial. The roots $\rho_{i}=\frac{1}{1+e^{ \pm \gamma}}$ of the delta function argument in eq. (2.12) are manifestly located inside the domain of the $x$-integration. The result of integrating out the delta function in eq. (2.12) is therefore

$$
\operatorname{Im} F_{1(\mathrm{a})}^{(1)}=-\pi \frac{C^{(1)}}{2 \epsilon}\left(\frac{\mu}{\Lambda}\right)^{2 \epsilon} \operatorname{coth} \gamma
$$

in agreement with eq. (2.5). The calculation for the diagram in figure $1(\mathrm{~b})$ is completely analogous, except that in this case $\int_{0}^{1} \mathrm{~d} x \delta\left(\left(x v_{1}-(1-x) v_{2}\right)^{2}\right)=0$, as both roots $\rho_{i}=\frac{1}{1-e^{ \pm \gamma}}$ are located outside the domain of integration. We therefore find a vanishing imaginary part, in agreement with eq. (2.4). We conclude that in both cases (a) and (b), the cutting prescription (2.11) produces the correct imaginary part. We introduce a graphical notation for the cutting prescription (2.11) in figure 3 .

It is natural to ask whether the imaginary part of eikonal diagrams can also be obtained from their momentum-space representation ${ }^{4}$

$$
F^{(1)}=i g^{2} C_{F} \mu^{2 \epsilon} \int \frac{\mathrm{d}^{D} k}{(2 \pi)^{D}} \frac{v_{1} \cdot v_{2}}{\left(k^{2}+i \eta\right)\left(v_{1} \cdot k+i \eta\right)\left(v_{2} \cdot k-i \eta\right)} .
$$

\footnotetext{
${ }^{4}$ The momentum-space representation in eq. (2.15) is straightforwardly obtained from the eikonal Feynman rules. By Schwinger parametrizing the eikonal propagators in eq. (2.15) and performing the resulting Fourier transform, one recovers the position-space representation in eq. (2.1).
} 


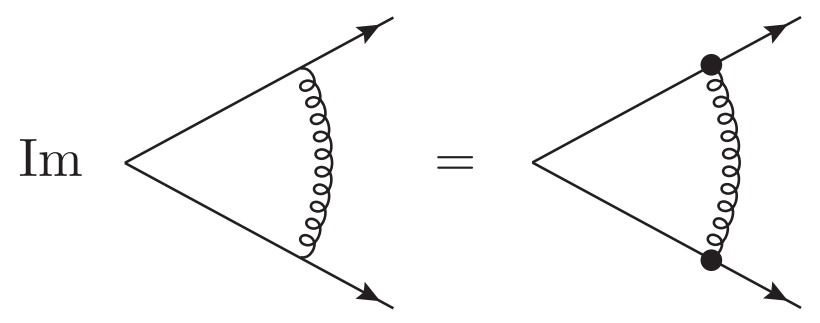

Figure 3. Graphical representation of a cut of an eikonal diagram in its position-space representation. The black dots represent the emission and absorption of a lightlike gauge boson.

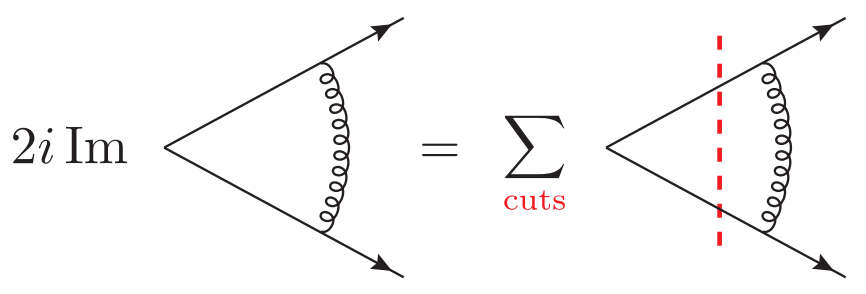

Figure 4. Graphical representation of cuts of eikonal diagrams in momentum space.

Such a cutting prescription was provided in ref. [21]. Here it was shown that the imaginary part of the one-loop diagram in eq. (2.15) may be obtained by replacing the two eikonal propagators by delta functions,

$$
\frac{1}{k \cdot v_{i} \pm i \eta} \stackrel{\text { cut }}{\longrightarrow} \mp 2 \pi i \theta\left(v_{i}^{0}\right) \delta\left(k \cdot v_{i}\right)
$$

This prescription can be thought of as the eikonal limit of the standard Cutkosky rules. It is illustrated in figure 4 above. More explicitly, applying the prescription (2.16) to eq. (2.15), the imaginary part is determined as follows,

$$
2 i \operatorname{Im} F^{(1)}=(2 \pi)^{2} i g^{2} C_{F} \theta\left(v_{1}^{0}\right) \theta\left(v_{2}^{0}\right)\left(v_{1} \cdot v_{2}\right) \mu^{2 \epsilon} \int \frac{\mathrm{d}^{D} k}{(2 \pi)^{D}} \frac{\delta\left(v_{1} \cdot k\right) \delta\left(v_{2} \cdot k\right)}{k^{2}+i \eta} .
$$

This representation of the imaginary part of the one-loop diagram motivates two remarks.

The first remark concerns the region of momentum space which gives rise to the imaginary part. Defining the light-cone variables $k^{ \pm} \equiv \frac{1}{\sqrt{2}}\left(k^{0} \pm k^{3}\right)$ and choosing the Lorentz frame in which the transverse components of the velocities vanish, $v_{i T}=0$, the support of the delta functions in eq. (2.17) is the region where the momentum of the exchanged gluon is maximally transverse,

$$
k_{T} \gg k^{+} \sim k^{-} \approx 0,
$$

which was identified in ref. [21] as the Glauber region [70]. This agrees with the discussion in section 1: the imaginary part of eikonal diagrams arises from the exchanges of Glauberregion gluons.

The second remark concerns the physical interpretation of applying the momentumspace cuts (2.16). By writing the delta functions in eq. (2.17) in terms of the plane-wave 


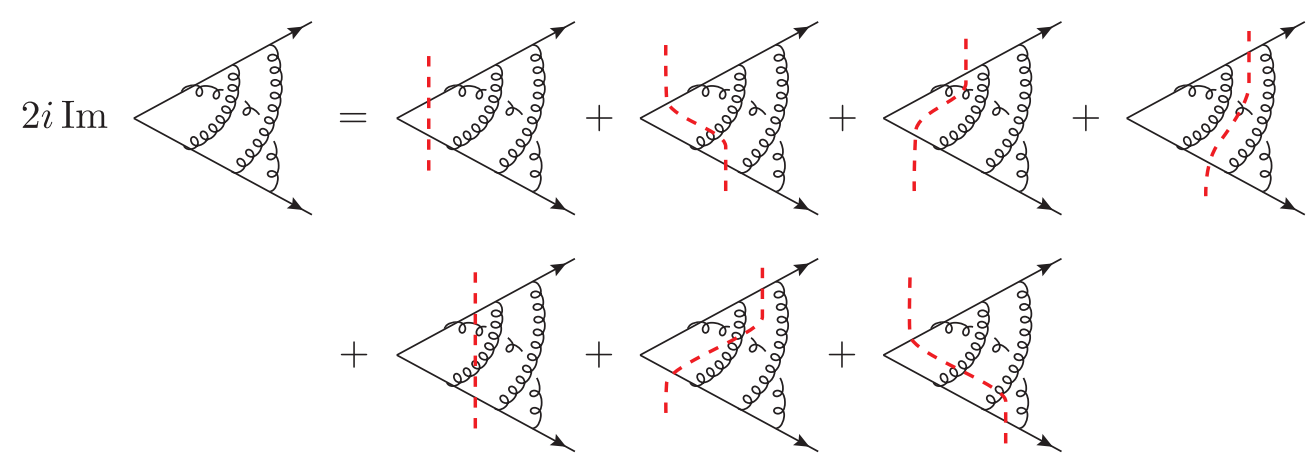

Figure 5. The non-vanishing momentum-space cuts of a non-planar three-loop ladder diagram. The cuts require the evaluation of two-, three- and four-particle phase-space integrals.

representation $\delta(A)=\frac{1}{2 \pi} \int_{-\infty}^{\infty} \mathrm{d} u e^{i u A}$ and performing the Fourier transform we find

$$
2 i \operatorname{Im} F^{(1)}=C^{(1)} \theta\left(v_{1}^{0}\right) \theta\left(v_{2}^{0}\right) \mu^{2 \epsilon} \int_{-\infty}^{\infty} \mathrm{d} t_{1} \int_{-\infty}^{\infty} \mathrm{d} t_{2} \frac{v_{1} \cdot v_{2}}{\left[-\left(t_{1} v_{1}-t_{2} v_{2}\right)^{2}+i \eta\right]^{1-\epsilon}}
$$

We observe that the resulting integration bounds compared to those of the uncut diagram in eq. (2.1) are extended according to $\int_{0}^{\infty} \mathrm{d} t_{i} \longrightarrow \int_{-\infty}^{\infty} \mathrm{d} t_{i}$. This state of affairs can be simply understood on physical grounds: as the hard partons have been put on shell through the cutting rule (2.16), they are now asymptotic states propagating from $t_{i}=-\infty$ to the interaction point.

We see that the position- and momentum-space representations of eikonal diagrams offer complementary points of view on the origin of their imaginary part. To summarize, in the position-space representation, the imaginary part is seen to arise from the exchanges of lightlike soft gauge bosons whose emission and absorption change the phases of the hard-parton states. In contrast, in momentum space, the imaginary part (related to the branch cut discontinuity through eq. (3.14)) arises from the two hard partons going on shell and exchanging Glauber gluons. Thus, the position- and momentum-space representations explain the origin of the imaginary part from the points of view of causality and unitarity, respectively.

The momentum-space cutting prescription in eq. (2.16) has the conceptual advantage of factoring eikonal diagrams into on-shell lower-loop and tree diagrams which in turn can be computed as independent objects. However, the resulting cut diagrams involve integrations over two-, three-, four-, ... particle phase space, as illustrated in figure 5. In practice, the evaluation of these phase-space integrals poses a substantial computational challenge which limits the applicability of the cut prescription (2.16) for obtaining imaginary parts.

As we shall see in section 3, in position space, eikonal diagrams without internal vertices take the form of iterated integrals. In this representation, their imaginary parts can therefore be straightforwardly obtained by applying the principal-value formula (2.10) recursively. 


\section{Position-space cuts of eikonal diagrams without internal vertices}

For completeness, in this section we review the derivation presented in ref. [1] of the imaginary part of $L$-loop eikonal diagrams without internal (i.e., three- or four-gluon) vertices to the leading order in the dimensional regulator $\epsilon$. We will interchangeably refer to these diagrams as ladder-type diagrams. The basic observation is that in position space these diagrams are iterated integrals, and as a result their imaginary part can be obtained by decomposing the real-line integrations into principal-value and delta function contributions.

In position space, an arbitrary $L$-loop eikonal diagram without internal vertices is composed of $L$ soft-gluon propagators, interchangeably referred to here as rungs. Each rung extends between the Wilson lines spanned by any two (possibly identical) external four-velocities $v_{1}, \ldots, v_{n}$ where $1 \leq n \leq L+1$. For the $j$ th rung we will denote these four-velocities by $v_{\ell_{j}}$ and $v_{r_{j}}$. We let $t_{i, k}$ denote the position of the $k$ th attachment on the Wilson line spanned by $v_{i}$, counting from the hard interaction vertex and outwards, so that $0 \leq t_{i, 1}<t_{i, 2}<\cdots<t_{i, N_{i}-1}<t_{i, N_{i}}$, where $N_{i}$ denotes the total number of soft-gluon attachments on the Wilson line. In addition, for the $j$ th rung, we let the variables $m_{j}$ and $n_{j}$ record the soft-gluon attachment numbers on the Wilson lines spanned by $v_{\ell_{j}}$ and $v_{r_{j}}$, respectively. The $L$-loop eikonal diagram is then defined as the $2 L$-fold iterated integral

$$
F^{(L)}=C^{(L)}\left(g \mu^{\epsilon}\right)^{2 L} \prod_{j=1}^{L} \int_{0}^{\infty} \mathrm{d} t_{\ell_{j}, m_{j}} \mathrm{~d} t_{r_{j}, n_{j}} \frac{\left(v_{\ell_{j}} \cdot v_{r_{j}}\right) \prod_{i=1}^{n} \prod_{k=0}^{N_{i}} \theta\left(t_{i, k+1}-t_{i, k}\right)}{\left[-\left(t_{\ell_{j}, m_{j}} v_{\ell_{j}}-t_{r_{j}, n_{j}} v_{r_{j}}\right)^{2}+i \eta\right]^{1-\epsilon}}
$$

where the kinematics-independent prefactor $C^{(L)}$ is determined by the color structure of the diagram and where it is implied that $t_{i, N_{i}+1} \equiv \infty$ and $t_{i, 0} \equiv 0$. Without loss of generality, we will assume that any rungs with both endpoints attached to the same Wilson line have been integrated out, and we suppress the resulting pole factors in $\epsilon$. (The additional factors produced by the integrations, involving epsilonic powers of the remaining variables, will not be of importance here, as our aim is to extract the imaginary part of $F^{(L)}$ to the leading order in $\epsilon$.)

To extract the imaginary part of $F^{(L)}$ from the integral representation in eq. (3.1) it turns out to be useful to perform a change of variables which leaves each soft propagator dependent on a single variable. To this end, we adopt a change of variables introduced in ref. [11]. The idea is to first express the attachment points of the $j$ th rung in terms of "polar" coordinates measuring the distance $\rho_{j}$ to the cusp (in units of the infrared cutoff $1 / \Lambda)$ and $x_{j}$ essentially measuring the emission angle of the soft gluon to the Wilson line, ${ }^{5}$

$$
\left(\begin{array}{c}
t_{\ell_{j}, m_{j}} \\
t_{r_{j}, n_{j}}
\end{array}\right)=\rho_{j}\left(\begin{array}{c}
x_{j} \\
1-x_{j}
\end{array}\right) \text { where }\left\{\begin{array}{l}
0 \leq \rho_{j}<\infty \\
0 \leq x_{j} \leq 1
\end{array}\right.
$$

\footnotetext{
${ }^{5}$ For a given rung, the two endpoints may of course be referred to interchangeably as left or right. However, for practical calculations, one particular choice may prove slightly more convenient. We refer to section 4 for examples.
} 
After this change of variables, the diagram takes the form

$$
F^{(L)}=C^{(L)}\left(g \mu^{\epsilon}\right)^{2 L} \prod_{j=1}^{L} \int_{0}^{\infty} \frac{\mathrm{d} \rho_{j}}{\rho_{j}^{1-2 \epsilon}} \int_{0}^{1} \mathrm{~d} x_{j} P_{\ell_{j} r_{j}}^{[\epsilon]}\left(x_{j}\right) \Theta(\boldsymbol{\rho}, \boldsymbol{x}),
$$

where the soft propagators are defined as

$$
P_{i j}^{[\epsilon]}(x) \equiv \frac{v_{i} \cdot v_{j}}{\left[-\left(x v_{i}-(1-x) v_{j}\right)^{2}+i \eta\right]^{1-\epsilon}},
$$

and where the nesting of the integrations is encoded in $\Theta$, defined through

$$
\left.\Theta(\boldsymbol{\rho}, \boldsymbol{x}) \equiv \prod_{i=1}^{n} \prod_{k=0}^{N_{i}} \theta\left(t_{i, k+1}-t_{i, k}\right)\right|_{\left(\begin{array}{c}
t_{\ell_{j}, m_{j}} \\
t_{r_{j}, n_{j}}
\end{array}\right)=\rho_{j}\left(\begin{array}{c}
x_{j} \\
1-x_{j}
\end{array}\right)} .
$$

We observe that the soft propagators' dependence on the radial coordinates $\rho_{j}$ has scaled out in eq. (3.3), and that each propagator now depends only on a single variable $x_{j}$. This turns out to be particularly advantageous for the purpose of extracting the imaginary part of the diagram, as this circumvents the need to divide a higher-dimensional domain of integration into subdomains characterized by supporting a specific number of propagator roots.

Now we extract the overall infrared divergence of the diagram by setting $\tau_{1} \equiv \rho_{1}$ and then applying the following sequence of $L-1$ substitutions

$$
\left(\begin{array}{c}
\tau_{1} \\
\rho_{2}
\end{array}\right)=\tau_{2}\left(\begin{array}{c}
y_{1} \\
1-y_{1}
\end{array}\right), \ldots,\left(\begin{array}{c}
\tau_{L-1} \\
\rho_{L}
\end{array}\right)=\tau_{L}\left(\begin{array}{c}
y_{L-1} \\
1-y_{L-1}
\end{array}\right) \quad \text { with } \quad\left\{\begin{array}{l}
0 \leq \tau_{j}<\infty \\
0 \leq y_{j} \leq 1
\end{array}\right.
$$

where the variables $\tau_{j}$ have the dimension of length and the $y_{j}$ are dimensionless. The $L$-loop eikonal diagram then becomes

$$
F^{(L)}=C^{(L)} \prod_{j=1}^{L} \int_{0}^{1} \mathrm{~d} x_{j} P_{\ell_{j} r_{j}}^{[\epsilon]}\left(x_{j}\right) K\left(x_{1}, \ldots, x_{L}\right),
$$

where the infrared divergence of the diagram is now absorbed into the kernel

$$
K\left(x_{1}, \ldots, x_{L}\right)=g^{2 L} \Gamma(2 L \epsilon)\left(\frac{\mu}{\Lambda}\right)^{2 L \epsilon} \prod_{j=1}^{L-1} \int_{0}^{1} \mathrm{~d} y_{j} y_{j}^{-1+2 j \epsilon}\left(1-y_{j}\right)^{-1+2 \epsilon} \Theta(\{\boldsymbol{y}, \boldsymbol{x}\}) .
$$

Here $\Theta(\{\boldsymbol{y}, \boldsymbol{x}\})$ denotes the result of applying the substitutions (3.6) to eq. (3.5). In analogy with section 2 , we have here regulated the infrared divergence in a gauge invariant way through the exponential damping factor $e^{-\Lambda \tau_{L}}$ with $\Lambda \ll 1$. Eq. (3.8) contains in addition any potential ultraviolet subdivergences of the diagram (generated by the nesting function $\Theta(\{\boldsymbol{y}, \boldsymbol{x}\}))$.

Having brought the $L$-loop eikonal diagram in the form (3.7), we now turn to extracting its imaginary part. Restricting our attention to the leading order in the dimensional regulator $\epsilon$, we will drop the dependence of the soft propagators on $\epsilon$,

$$
F^{(L)}=C^{(L)} \prod_{j=1}^{L} \int_{0}^{1} \mathrm{~d} x_{j} P_{\ell_{j} r_{j}}^{[0]}\left(x_{j}\right) K\left(x_{1}, \ldots, x_{L}\right)+\mathcal{O}\left(\epsilon^{-d+1}\right),
$$

where $d$ denotes the degree of divergence of the diagram, $F^{(L)} \sim \frac{1}{\epsilon^{d}} \times$ (finite). 


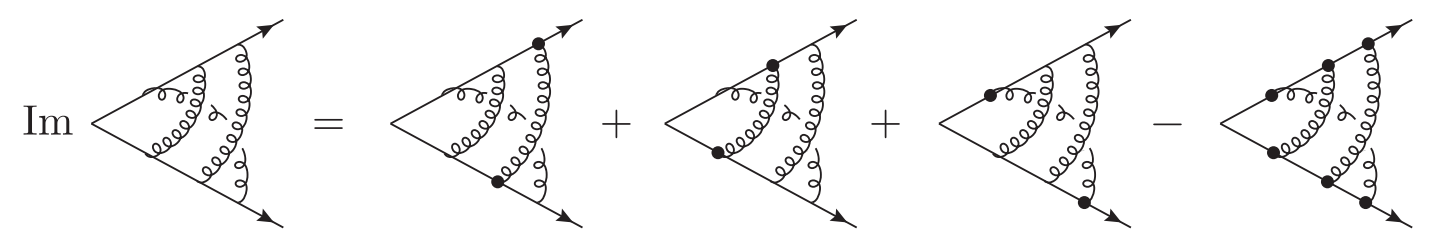

Figure 6. Schematic illustration of the formula (3.12) for the imaginary part of an eikonal diagram without internal vertices. The black dots at the endpoints of a soft-gluon propagator denote that the propagator has been cut - that is, replaced by a delta function. It is implied that the integrals over the attachment points of uncut soft propagators are principal-value integrals. The relative signs of the diagrams are determined by the factor $i^{p-1}$; each individual diagram displayed here corresponds to the action of the cutting operator (3.11) on the eikonal diagram.

To compute the imaginary part of eq. (3.9), we start by observing that eq. (3.8) is manifestly purely real. As a result, the Feynman $i \eta$ 's are the only source of imaginary parts of eq. (3.9). Each of the $x_{j}$-integration paths can therefore be decomposed into a principal-value part and small semicircles around the propagator poles. Given that the integrand takes purely imaginary values in the regions close to the poles and is real-valued on the remaining domain of integration, the resulting $2^{L}$ terms (which each involve $L$ integrations) will be either purely real or purely imaginary.

To collect the imaginary contributions, we define the cut propagator

$$
\Delta_{i j}(x) \equiv-\pi v_{i} \cdot v_{j} \delta\left(\left(x v_{i}-(1-x) v_{j}\right)^{2}\right),
$$

and furthermore $p$-fold cutting operator

$$
\mathrm{Cut}_{x_{i_{1}}, \ldots, x_{i_{p}}} F^{(L)}=\prod_{\substack{j=1 \\ j \neq i_{1}, \ldots, i_{p}}}^{n} \mathrm{PV} \int_{0}^{1} \mathrm{~d} x_{j} P\left(x_{j}\right) \prod_{k=1}^{p} \int_{0}^{1} \mathrm{~d} x_{i_{k}} \Delta\left(x_{i_{k}}\right) K\left(x_{1}, \ldots, x_{L}\right) .
$$

The action of this operator is to replace the $p$ propagators that depend on the specified variables by delta functions and to place a principal-value prescription on the integrals over the remaining variables. To simplify notation, we here dropped the indices on the (cut) propagators: $P\left(x_{j}\right) \equiv P_{\ell_{j} r_{j}}^{[0]}\left(x_{j}\right)$ and $\Delta\left(x_{j}\right) \equiv \Delta_{\ell_{j} r_{j}}\left(x_{j}\right)$.

The imaginary part of any $L$-loop eikonal diagram without internal vertices can then be written, to the leading order in $\epsilon$,

$$
\operatorname{Im} F^{(L)}=\sum_{\substack{p=1 \\ p \text { odd }}}^{L} \sum_{\substack{i_{1}, \ldots, i_{p}=1 \\ i_{1}<\cdots<i_{p}}}^{L} i^{p-1} \operatorname{Cut}_{x_{i_{1}}, \ldots, x_{i_{p}}} F^{(L)} .
$$

This is the central formula of our approach [1]. The formula (3.12) is illustrated schematically for a generic ladder diagram in figure 6 above.

We note that the decomposition of the line integrations in eq. (3.9) into principalvalue and delta function contributions immediately shows that the imaginary part of the integrated expression for the eikonal diagram will have transcendentality weight one less 
than the real part. This follows from the fact that the delta functions will map the rational integrand to a rational expression after being integrated out. Thus, compared to the realpart contribution with $L$ principal-value integrals, the weight is dropped by one.

It is natural to ask about the relation of the imaginary part of the eikonal diagram to the discontinuities in its various kinematic channels. This in turn leads us to ask for an appropriate set of variables in terms of which to express integrated results. A good choice of variables turns out to be given by the exponentials of the cusp angles,

$$
\chi_{i j} \equiv e^{-\gamma_{i j}}
$$

with the cusp angles defined through $\cosh \gamma_{i j}=\left|v_{i} \cdot v_{j}\right|$. Expressed in terms of the $\chi_{i j}$ variables, the eikonal diagram has branch cuts located on the real line and satisfies Schwarz reflection, $F^{(L)}\left(\overline{\chi_{i j}}\right)=\overline{F^{(L)}\left(\chi_{i j}\right)}$. As a result, the discontinuities of the diagram give rise to the imaginary part through the relation

$$
2 i \operatorname{Im} F^{(L)}(\chi)=\sum_{j=1}^{L} \theta\left(v_{\ell_{j}} \cdot v_{r_{j}}\right) \operatorname{\chi isc}_{\chi_{\ell_{j} r_{j}}} F^{(L)}(\boldsymbol{\chi}) .
$$

Here, the step functions account for the fact that the imaginary part has vanishing contributions from channels with space-like kinematics $v_{\ell_{j}} \cdot v_{r_{j}}<0$. (This follows from the fact that propagators stretched between mutually space-like eikonal lines have vanishing cuts, as will be explained below eq. (3.16).) We will see an explicit example of this in section 4.3 where we study a diagram that depends on two distinct cusp angles in purely time-like as well as mixed time- and space-like kinematics.

In section 4 we will work out examples of how eq. (3.12) is used in practice to compute the imaginary part of ladder-type eikonal diagrams. To this end it will be useful to record the following partial-fractioned expressions, setting $\chi \equiv \chi_{i j}$,

$$
\begin{aligned}
& P_{i j}^{[0]}(x)=\frac{R(\chi)}{2}\left(\frac{1}{x-\rho_{1}+i \eta}-\frac{1}{x-\rho_{2}-i \eta}\right) \\
& \Delta_{i j}(x)=-\pi \frac{R(\chi)}{2}\left(\delta\left(x-\rho_{1}\right)+\delta\left(x-\rho_{2}\right)\right),
\end{aligned}
$$

where the prefactor is the rational expression $R(\chi)=\frac{1+\chi^{2}}{1-\chi^{2}}=\operatorname{coth} \gamma_{i j}$, and the denominator roots are given by

$$
\left(\rho_{1}, \rho_{2}\right)= \begin{cases}\left(\frac{\chi}{\chi+1}, \frac{1}{\chi+1}\right) & \text { for } v_{i} \cdot v_{j} \equiv \cosh \gamma_{i j}>0 \\ \left(\frac{\chi}{\chi-1}, \frac{1}{1-\chi}\right) & \text { for } v_{i} \cdot v_{j} \equiv-\cosh \gamma_{i j}<0\end{cases}
$$

We note that in the upper case of eq. (3.16), the roots satisfy $0<\rho_{1}<\frac{1}{2}<\rho_{2}<1$, whereas in the lower case they satisfy $\rho_{1}<0<1<\rho_{2}$. Since the delta functions in eq. (3.11) are integrated over the interval $[0,1]$, we may thus infer that the eikonal diagram will only have contributions to its imaginary part from channels with time-like kinematics $v_{i} \cdot v_{j}>0$, as encoded in eq. (3.14). This is in agreement with the causality considerations of section 2. 
In section 4 we will make extensive use of the fact that the result for an eikonal diagram in time-like kinematics can be immediately obtained from the space-like result by analytic continuation of the cusp angle. To see this, let us first recall that the soft propagator takes the same form (3.4) in space- and time-like kinematics when expressed in terms of $v_{i} \cdot v_{j}$, owing to our convention that all velocity vectors are outgoing. However, once expressed in terms of the relative angle $\gamma \equiv \gamma_{i j}$, it takes the respective forms

$$
P_{i j}^{[\epsilon]}(x)= \begin{cases}-\cosh \gamma\left[-x^{2}-(1-x)^{2}-2 x(1-x) \cosh \gamma\right]^{-1+\epsilon} & \text { for } v_{i} \cdot v_{j} \equiv-\cosh \gamma<0 \\ \cosh \gamma\left[-x^{2}-(1-x)^{2}+2 x(1-x) \cosh \gamma+i \eta\right]^{-1+\epsilon} & \text { for } v_{i} \cdot v_{j} \equiv \cosh \gamma>0\end{cases}
$$

where we dropped the $i \eta$ in the space-like case, as the propagator roots are located outside the range $[0,1]$ of $x$. Comparison of these expressions shows that we can map space-like to time-like kinematics by means of the analytic continuation

$$
-\cosh \gamma \longrightarrow \cosh \gamma+i \eta,
$$

or equivalently, in terms of $\chi \equiv e^{-\gamma}$,

$$
\chi \longrightarrow-\frac{1}{\chi}-i \eta
$$

\section{Examples}

The aim of this section is to apply the formalism reviewed in section 3 to compute the imaginary part of a number of ladder-type eikonal diagrams. The main point to be addressed here concerns the evaluation of the principal-value integrals involved in the $p$-fold cuts in eq. (3.11).

\subsection{The non-planar two-loop ladder diagram}

As a first example we will consider the non-planar two-loop ladder diagram, illustrated in figure 7 below. This diagram contains no ultraviolet subdivergence and therefore only has a simple pole in the dimensional regulator $\epsilon$. In agreement with the observations at the end of section 3, the diagram will only have an imaginary part for time-like kinematics $v_{1} \cdot v_{2}>0$. We therefore restrict our attention to this case. Since the diagram contains only one cusp angle, we will drop the subscripts for convenience and define $\cosh \gamma \equiv v_{1} \cdot v_{2}$ as well as $\chi \equiv e^{-\gamma}$.

The non-planar two-loop ladder diagram has the position-space representation

$$
F^{(2)}=C^{(2)} \mu^{4 \epsilon} \int_{0}^{\infty} \frac{\mathrm{d} t_{1,1} \mathrm{~d} t_{1,2} \mathrm{~d} t_{2,1} \mathrm{~d} t_{2,2} \theta\left(t_{1,2}-t_{1,1}\right) \theta\left(t_{2,2}-t_{2,1}\right)\left(v_{1} \cdot v_{2}\right)^{2}}{\left[-\left(t_{1,1} v_{1}-t_{2,2} v_{2}\right)^{2}+i \eta\right]^{1-\epsilon}\left[-\left(t_{1,2} v_{1}-t_{2,1} v_{2}\right)^{2}+i \eta\right]^{1-\epsilon}},
$$

where the prefactor is given by $C^{(2)}=-\frac{g^{4} C_{F}}{2 N} \frac{\Gamma^{2}(D / 2-1)}{16 \pi^{D}}$. To compute the imaginary part of this diagram, our first task is to write it in the form of eq. (3.7). This is achieved through the change of variables in eq. (3.2), followed by that in eq. (3.6),

$$
\left(\begin{array}{c}
t_{1,1} \\
t_{2,2}
\end{array}\right)=\lambda\left(\begin{array}{c}
x \\
1-x
\end{array}\right), \quad\left(\begin{array}{c}
t_{1,2} \\
t_{2,1}
\end{array}\right)=\sigma\left(\begin{array}{c}
y \\
1-y
\end{array}\right) \quad \text { followed by } \quad\left(\begin{array}{c}
\lambda \\
\sigma
\end{array}\right)=\beta\left(\begin{array}{c}
t \\
1-t
\end{array}\right) .
$$




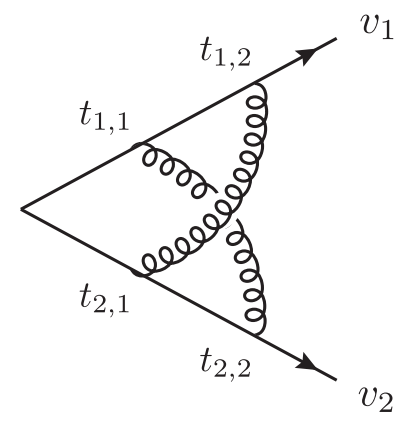

Figure 7. The non-planar two-loop ladder diagram.

After these transformations the diagram takes the desired form,

$$
F^{(2)}=C^{(2)} \int_{0}^{1} \mathrm{~d} x \int_{0}^{1} \mathrm{~d} y P_{12}^{[\epsilon]}(x) P_{12}^{[\epsilon]}(y) K(x, y),
$$

where the kernel $K(x, y)$, upon the additional change of variable $u=\frac{t}{1-t}$, is given by

$$
K(x, y)=\mu^{4 \epsilon} \int_{0}^{\infty} \frac{\mathrm{d} \beta e^{-\Lambda \beta}}{\beta^{1-4 \epsilon}} \int_{0}^{\infty} \mathrm{d} u u^{-1+2 \epsilon}(u+1)^{-4 \epsilon} \theta\left(\frac{y}{x}-u\right) \theta\left(u-\frac{1-y}{1-x}\right) .
$$

By comparing eqs. (4.1) and (4.3), we see that the effect of the first two transformations of eq. (4.2) is to leave each soft propagator dependent on a single variable. The effect of the last change of variable is to extract the overall infrared divergence of the diagram.

To facilitate the computation of the cuts in eq. (3.12) we will first evaluate the integral $K(x, y)$. The $u$-integral in eq. (4.4) may be performed in terms of the ${ }_{2} F_{1}$ hypergeometric function. The primitive has the $\epsilon$-expansion

$$
f(u)=\frac{u^{2 \epsilon}}{2 \epsilon}{ }_{2} F_{1}(2 \epsilon, 4 \epsilon ; 1+2 \epsilon ;-u)=\frac{u^{2 \epsilon}}{2 \epsilon}\left(1+\mathcal{O}\left(\epsilon^{2}\right)\right)=\frac{1}{2 \epsilon}+\log u+\mathcal{O}(\epsilon),
$$

and so $K(x, y)$ has the $\epsilon$-expansion

$$
\begin{aligned}
K(x, y) & =\Gamma(4 \epsilon)\left(\frac{\mu}{\Lambda}\right)^{4 \epsilon} \theta(y-x)\left[f\left(\frac{y}{x}\right)-f\left(\frac{1-y}{1-x}\right)\right] \\
& =\frac{1}{4 \epsilon}\left(\frac{\mu}{\Lambda}\right)^{4 \epsilon} \theta(y-x)\left(\log \frac{y}{x}-\log \frac{1-y}{1-x}\right)+\mathcal{O}\left(\epsilon^{0}\right) .
\end{aligned}
$$

Substituting this result for $K(x, y)$ into eq. (4.3), we can write the non-planar two-loop ladder diagram in the convenient form

$$
F^{(2)}=\frac{C^{(2)}}{4 \epsilon}\left(\frac{\mu}{\Lambda}\right)^{4 \epsilon} \mathcal{F}^{(2)}
$$

where $\mathcal{F}^{(2)}$ is finite, given to leading order in $\epsilon$ as,

$$
\mathcal{F}^{(2)}=\int_{0}^{1} \mathrm{~d} x \int_{0}^{1} \mathrm{~d} y P_{12}^{[0]}(x) P_{12}^{[0]}(y)(\theta(y-x)-\theta(x-y)) \log \frac{y}{x} .
$$


Here we have dropped the dependence of the soft propagators on $\epsilon$ and furthermore rewritten the integrand of $\mathcal{F}^{(2)}$ to make its symmetry under $x \longleftrightarrow y$ manifest. (This was achieved by changing variables $(x, y) \mapsto(1-x, 1-y)$ on the second term $\log \frac{1-y}{1-x}$ arising from eq. (4.6).)

As the prefactor of $\mathcal{F}^{(2)}$ in eq. (4.7) is real, it factors out on both sides of eq. (3.12), yielding the formula

$$
\operatorname{Im} \mathcal{F}^{(2)}=\mathrm{Cut}_{x} \mathcal{F}^{(2)}+\mathrm{Cut}_{y} \mathcal{F}^{(2)}
$$

where we recall that $\mathrm{Cut}_{x_{i}}$ is defined in eq. (3.11) and replaces the propagator depending on the specified variable by a delta function and places a principal-value prescription on the integral over the remaining variable. The two cuts on the right-hand side are equal because the integrand of eq. (4.8) is symmetric under the interchange of $x$ and $y$. (This also follows from the $v_{1} \longleftrightarrow v_{2}$ symmetry of the original diagram.) Thus, it suffices to compute $\mathrm{Cut}_{x} \mathcal{F}^{(2)}$, given by

$$
\mathrm{Cut}_{x} \mathcal{F}^{(2)}=\int_{0}^{1} \mathrm{~d} x \mathrm{PV} \int_{0}^{1} \mathrm{~d} y \Delta_{12}(x) P_{12}^{[0]}(y)(\theta(y-x)-\theta(x-y)) \log \frac{y}{x} .
$$

Inserting the partial-fractioned expressions for $\Delta_{12}(x)$ and $P_{12}^{[0]}(y)$ given in eq. (3.15) and performing the trivial integral over $x$ produces

$$
\operatorname{Cut}_{x} \mathcal{F}^{(2)}=-\frac{\pi}{4} R(\chi)^{2} \sum_{k=1}^{2} \operatorname{PV}\left(\int_{\rho_{k}}^{1} \mathrm{~d} y-\int_{0}^{\rho_{k}} \mathrm{~d} y\right)\left(\frac{1}{y-\rho_{1}+i \eta}-\frac{1}{y-\rho_{2}-i \eta}\right) \log \frac{y}{\rho_{k}},
$$

where the propagator roots $\rho_{1,2}$ are given in the upper part of eq. (3.16).

We are now confronted with the task of evaluating principal-value integrals. As such integrals do not immediately take the form of iterated integrals, our strategy for evaluation will be to write them as differences of iterated integrals, which in turn are readily expressible in terms of multiple polylogarithms. The basic observation is that the principal-value integral equals the corresponding full integral minus the imaginary part of the latter, cf. eq. (2.10).

As a simple illustration, let us consider the evaluation of the following principal-value integral,

$$
\mathrm{PV} \int_{0}^{1} \frac{\mathrm{d} y}{y-\rho_{1}+i \eta}=\int_{0}^{1} \frac{\mathrm{d} y}{y-\rho_{1}+i \eta}-i \operatorname{Im} \int_{0}^{1} \frac{\mathrm{d} y}{y-\rho_{1}+i \eta} .
$$

The full integral evaluates to $G\left(\rho_{1} ; 1\right)$ by definition, cf. eqs. (A.1)-(A.2). Its imaginary part arises from the pole of the integrand and is extracted by localizing the integration variable,

$$
\operatorname{Im} \int_{0}^{1} \frac{\mathrm{d} y}{y-\rho_{1}+i \eta}=-\pi \int_{0}^{1} \mathrm{~d} y \delta\left(y-\rho_{1}\right)=-\pi,
$$

where we used in the last step that the pole is located inside the range of integration, in agreement with the discussion below eq. (3.16). Thus we arrive at

$$
\mathrm{PV} \int_{0}^{1} \frac{\mathrm{d} y}{y-\rho_{1}+i \eta}=G\left(\rho_{1} ; 1\right)+\pi i
$$


In this simple example we could have computed the real part more directly,

$$
\mathrm{PV} \int_{0}^{1} \frac{\mathrm{d} y}{y-\rho_{1}}=\operatorname{Re} G\left(\rho_{1} ; 1\right)=\operatorname{Re} \log \left(1-\frac{1}{\rho_{1}}\right)=\log \left|1-\frac{1}{\rho_{1}}\right|=\log \left(\frac{1}{\rho_{1}}-1\right) .
$$

However, an extension of this direct approach to higher-weight cases requires the use of a sequence of functional identities which in practice is case-dependent and thus not applicable in a systematic way. In contrast, the above method relies only on a construction of the imaginary part which can be derived systematically as demonstrated in appendix A.

Returning to eq. (4.11) and evaluating the principal-value integrals following the steps outlined above, the result for the cut is readily expressed in terms of multiple polylogarithms,

$$
\begin{aligned}
\operatorname{Cut}_{x} \mathcal{F}^{(2)}=-\frac{\pi}{4} R(\chi)^{2}( & -2 G\left(\rho_{2}, 0 ; 1\right)+2 G\left(\rho_{1}, 0 ; 1\right)-2 G\left(0, \rho_{2} ; \rho_{2}\right)-2 G\left(0, \rho_{2} ; \rho_{1}\right) \\
& +2 G\left(0, \rho_{1} ; \rho_{2}\right)+2 G\left(0, \rho_{1} ; \rho_{1}\right)+G\left(0 ; \rho_{2}\right) G\left(\rho_{2} ; 1\right)+G\left(0 ; \rho_{1}\right) G\left(\rho_{2} ; 1\right) \\
& \left.-G\left(0 ; \rho_{2}\right) G\left(\rho_{1} ; 1\right)-G\left(0 ; \rho_{1}\right) G\left(\rho_{1} ; 1\right)-2 \pi i G\left(0 ; \frac{\rho_{1}}{\rho_{2}}\right)\right),
\end{aligned}
$$

where we refer to eqs. (A.1)-(A.2) for definitions. In this expression, the multiple polylogarithms depend on the propagator roots $\rho_{k}(\chi)$ through both their indices and their arguments. This expression can in turn be rewritten in terms of polylogarithms with constant indices by exploiting the Hopf algebra structure of multiple polylogarithms, which encodes the plethora of functional identities within this class of functions [71-76]. Utilizing this algebraic structure, we have implemented the steps required to achieve the desired functional form as a general algorithm. We refer to appendix B for further details. The algorithm leaves a simplified form of eq. (4.16) expressed in terms of harmonic polylogarithms which, using eq. (A.5), can be simplified further into classical polylogarithms,

$$
\begin{aligned}
\operatorname{Cut}_{x} \mathcal{F}^{(2)} & =-\pi R(\chi)^{2}\left(-G(0,1 ; \chi)+G(0,0 ; \chi)-G(0,-1 ; \chi)-\frac{1}{2} \zeta_{2}\right) \\
& =-\frac{\pi}{2} R(\chi)^{2}\left(\operatorname{Li}_{2}\left(\chi^{2}\right)+\log ^{2} \chi-\zeta_{2}\right)
\end{aligned}
$$

With this result, we can now immediately obtain the imaginary part of the two-loop ladder from eq. (4.9), recalling that the two cuts are equal. We find

$$
\operatorname{Im} \mathcal{F}^{(2)}=-\pi R(\chi)^{2}\left(\operatorname{Li}_{2}\left(\chi^{2}\right)+\log ^{2} \chi-\zeta_{2}\right),
$$

and we recall that multiplying the infrared pole cf. eq. (4.7) onto both sides of eq. (4.18) gives the imaginary part of the original diagram $F^{(2)}$. This completes the evaluation of the imaginary part of the non-planar two-loop ladder diagram to the leading order in $\epsilon$.

As a crosscheck of the result in eq. (4.18), we can alternatively compute the imaginary part of the non-planar two-loop ladder by evaluating the diagram for space-like kinematics $v_{1} \cdot v_{2}<0$, in which case it will be purely real (cf. the discussion at the end of section 3 ), 
and subsequently perform the analytic continuation to time-like kinematics. We refer to the end of section 3 for a more detailed discussion of analytic continuations.

To the leading order in $\epsilon$, the two-loop ladder is given by eq. (4.8), although we must bear in mind that for space-like kinematics the propagator roots $\rho_{k}$ are given by the lower case of eq. (3.16). Inserting into eq. (4.8) the expressions for $P_{12}^{[0]}$ given in eq. (3.15), the diagram readily evaluates into multiple polylogarithms,

$$
\widetilde{\mathcal{F}}^{(2)}=\frac{R(\chi)^{2}}{2}\left(G\left(\rho_{1}, 0, \rho_{1} ; 1\right)-G\left(\rho_{1}, 0, \rho_{2} ; 1\right)-G\left(\rho_{2}, 0, \rho_{1} ; 1\right)+G\left(\rho_{2}, 0, \rho_{2} ; 1\right)\right) .
$$

The tilde on the left-hand side serves to remind us that the expression for the diagram on the right-hand side is valid for space-like kinematics. We can use the algorithm in appendix B to recast this representation in terms of polylogarithms with constant indices. In fact, the two-loop ladder diagram can be expressed in terms of classical polylogarithms, ${ }^{6}$

$$
\begin{aligned}
\widetilde{\mathcal{F}}^{(2)} & =R(\chi)^{2}\left(2 G(0,1,0 ; \chi)-2 G(0,0,0 ; \chi)+2 G(0,-1,0 ; \chi)-\zeta_{2} G(0 ; \chi)-\zeta_{3}\right) \\
& =R(\chi)^{2}\left(\operatorname{Li}_{3}\left(\chi^{2}\right)-\log \chi \operatorname{Li}_{2}\left(\chi^{2}\right)-\frac{1}{3} \log ^{3} \chi-\zeta_{2} \log \chi-\zeta_{3}\right) .
\end{aligned}
$$

We can now find the result for the two-loop ladder diagram in time-like kinematics by performing the analytic continuation $\chi \rightarrow-1 / \chi-i \eta$ on eq. (4.20). Under the analytic continuation, the rational function $R(\chi)$ picks up a minus sign, while the polylogarithms transform according to

$$
\begin{aligned}
\log \chi & \rightarrow-\log \chi-\pi i \\
\operatorname{Li}_{2}\left(\chi^{2}\right) & \rightarrow-\operatorname{Li}_{2}\left(\chi^{2}\right)-2 \log ^{2} \chi+2 \zeta_{2}-2 \pi i \log \chi \\
\operatorname{Li}_{3}\left(\chi^{2}\right) & \rightarrow \operatorname{Li}_{3}\left(\chi^{2}\right)+\frac{4}{3} \log ^{3} \chi-4 \zeta_{2} \log \chi+2 \pi i \log ^{2} \chi .
\end{aligned}
$$

Applying these replacements to eq. (4.20) we find the following result for the non-planar two-loop ladder with time-like kinematics,

$$
\begin{gathered}
\mathcal{F}^{(2)}=R(\chi)^{2}\left(\operatorname{Li}_{3}\left(\chi^{2}\right)-\log \chi \operatorname{Li}_{2}\left(\chi^{2}\right)-\frac{1}{3} \log ^{3} \chi+5 \zeta_{2} \log \chi-\zeta_{3}\right. \\
\left.-i \pi\left(\operatorname{Li}_{2}\left(\chi^{2}\right)+\log ^{2} \chi-\zeta_{2}\right)\right) .
\end{gathered}
$$

We observe that the imaginary part of eq. (4.22) agrees with the result found in eq. (4.18), as expected. We conclude that the cutting prescription for the two-loop ladder stated in eq. (4.9) produces the correct imaginary part. The cutting prescription (4.9) is illustrated in figure 8.

\footnotetext{
${ }^{6}$ Note that in the representation in the second line of eq. (4.20), the polylogarithms and logarithms have the respective arguments $\chi^{2}$ and $\chi$. This form has the advantage of being particularly compact as well as convenient for the purpose of performing the analytic continuation $\chi \rightarrow-1 / \chi-i \eta$ which then maps the arguments of the (poly)logarithms to the vicinity of the branch cuts. (In general, computer algebra software, such as Mathematica augmented with the package HPL $[77,78]$, does not detect the $2 \pi$ monodromy of $\log \left(\chi^{2}\right)$ after tracing out a complete circle around the branch point.)
} 


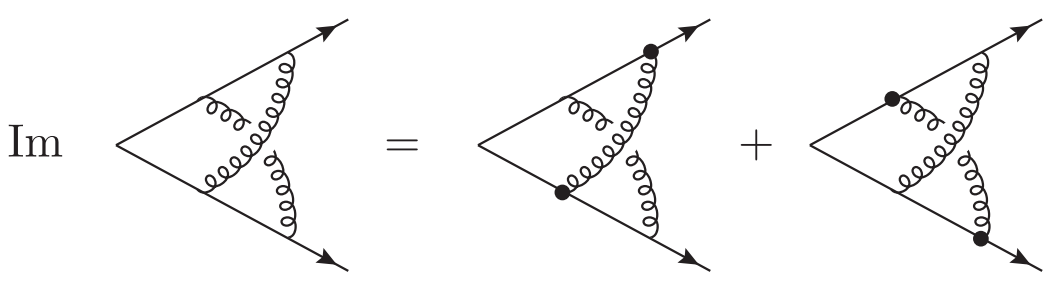

Figure 8. Graphical representation of the cutting prescription for the non-planar two-loop ladder stated in eq. (4.9). The black dots at the endpoints of a soft-gluon propagator indicate that the propagator has been cut; i.e., replaced by a delta function. It is implied that the integrals over the attachment points of uncut soft propagators are principal-value integrals.

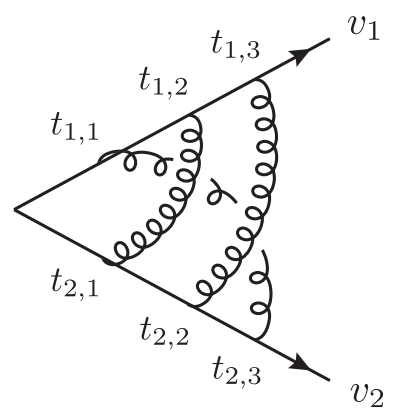

Figure 9. A three-loop non-planar ladder diagram.

\subsection{Three-loop non-planar ladder diagram}

To demonstrate that the principal-value integrals involved in the $p$-fold cuts in eq. (3.11) can indeed be evaluated in non-trivial cases, we consider in this section the three-loop ladder diagram illustrated in figure 9. This diagram also represents an example of an eikonal diagram with multiple-cut contributions to its imaginary part (in the case at hand, a triple cut). As in section 4.1, we take $\cosh \gamma \equiv v_{1} \cdot v_{2}>0$, in order to have a non-vanishing imaginary part, and set $\chi \equiv e^{-\gamma}$.

The position-space representation of the diagram in figure 9 takes the form

$$
F^{(3)}=C^{(3)} \mu^{6 \epsilon} \int_{0}^{\infty}\left(\prod_{i=1}^{2} \prod_{j=1}^{3} \mathrm{~d} t_{i, j}\right) \frac{\prod_{i, j=1}^{2} \theta\left(t_{i, j+1}-t_{i, j}\right)\left(v_{1} \cdot v_{2}\right)^{3}}{D\left(t_{1,1}, t_{2,3}\right) D\left(t_{1,2}, t_{2,1}\right) D\left(t_{1,3}, t_{2,2}\right)},
$$

where $D\left(t_{1}, t_{2}\right)=\left[-\left(t_{1} v_{1}-t_{2} v_{2}\right)^{2}+i \eta\right]^{1-\epsilon}$. To compute the imaginary part of this diagram from eq. (3.12), our first task is to bring it into the form of eq. (3.7). This is achieved through the changes of variables in eq. (3.2) (with $t_{\ell_{j}, m_{j}}=t_{1, j}$ ), followed by the sequence of substitutions in eq. (3.6), setting $\left(x_{1}, x_{2}, x_{3}\right)=(x, y, z)$ and $\left(y_{1}, y_{2}\right)=(t, u)$ for convenience.

After these transformations, the diagram takes the form

$$
F^{(3)}=C^{(3)} \int_{0}^{1} \mathrm{~d} x \mathrm{~d} y \mathrm{~d} z P_{12}^{[\epsilon]}(x) P_{12}^{[\epsilon]}(y) P_{12}^{[\epsilon]}(z) K(x, y, z),
$$


where the kernel is given by

$$
\begin{aligned}
K(x, y, z)= & \Gamma(6 \epsilon)\left(\frac{\mu}{\Lambda}\right)^{6 \epsilon} \int_{0}^{1} \mathrm{~d} t t^{-1+2 \epsilon}(1-t)^{-1+2 \epsilon} \int_{0}^{1} \mathrm{~d} u u^{-1+4 \epsilon}(1-u)^{-1+2 \epsilon} \\
& \times \theta\left(\frac{z}{y}-\frac{(1-t) u}{1-u}\right) \theta\left(\frac{y}{x}-\frac{t}{1-t}\right) \theta\left(\frac{t u}{1-u}-\frac{1-z}{1-x}\right) \theta\left(\frac{1-z}{1-y}-\frac{(1-t) u}{1-u}\right) .
\end{aligned}
$$

The arguments of the step functions simplify after rescaling the integration variables according to $\frac{t}{1-t} \mapsto t$ and $\frac{u}{1-u} \mapsto u$. As we are interested in computing the imaginary part of $F^{(3)}$ only to the leading order in $\epsilon$, we may set $\epsilon$ to zero in the $u$-integral. Performing the $t$-integral in terms of hypergeometric functions and subsequently expanding in $\epsilon$, we find the expression

$$
\begin{aligned}
K(x, y, z) & =\frac{1}{6 \epsilon}\left(\frac{\mu}{\Lambda}\right)^{6 \epsilon}\left[\theta(y-z) \theta(z-x) \frac{1}{2} \log ^{2}\left(\frac{1-x}{x} \frac{z}{1-z}\right)+(y \longleftrightarrow z)\right] \\
& \equiv \frac{1}{6 \epsilon}\left(\frac{\mu}{\Lambda}\right)^{6 \epsilon} \mathcal{K}(x, y, z),
\end{aligned}
$$

valid to the leading order in $\epsilon$. We observe that eq. (4.26) is symmetric under the interchange of $y$ and $z$; hence the integrand of the full diagram in eq. (4.24) is as well. (In other words, interchanging the two parallel gluon lines in figure 9 leaves the diagram invariant.) This observation implies that $\mathrm{Cut}_{y} F^{(3)}=\mathrm{Cut}_{z} F^{(3)}$ and thereby reduces the number of independent cuts to be computed.

Substituting eq. (4.26) into eq. (4.24) we can write the non-planar three-loop ladder diagram in the convenient form

$$
F^{(3)}=\frac{C^{(3)}}{6 \epsilon}\left(\frac{\mu}{\Lambda}\right)^{6 \epsilon} \mathcal{F}^{(3)}
$$

where $\mathcal{F}^{(3)}$ is finite, given to leading order in $\epsilon$ as

$$
\mathcal{F}^{(3)}=\int_{0}^{1} \mathrm{~d} x \mathrm{~d} y \mathrm{~d} z P_{12}^{[0]}(x) P_{12}^{[0]}(y) P_{12}^{[0]}(z) \mathcal{K}(x, y, z) .
$$

As the prefactor of $\mathcal{F}^{(3)}$ in eq. (4.27) is real, it factors out on both sides of eq. (3.12), yielding the formula

$$
\operatorname{Im} \mathcal{F}^{(3)}=\operatorname{Cut}_{x} \mathcal{F}^{(3)}+\operatorname{Cut}_{y} \mathcal{F}^{(3)}+\mathrm{Cut}_{z} \mathcal{F}^{(3)}-\mathrm{Cut}_{x, y, z} \mathcal{F}^{(3)} .
$$

The appearance of a multiple-cut contribution represents a new feature for diagrams with more than two loops. Incidentally, the diagram $F^{(3)}$ is the only three-loop ladder diagram with an $\mathcal{O}\left(\frac{1}{\epsilon}\right)$ divergence that has a non-vanishing triple cut. This point is illustrated in figure 10. The diagram $F^{(3)}$ therefore provides an excellent example to demonstrate a multiple-cut contribution.

Let us start by evaluating this triple cut: as all three integrations are localized by delta functions, the cut is immediately computed,

$$
\begin{aligned}
\operatorname{Cut}_{x, y, z} \mathcal{F}^{(3)} & =\int_{0}^{1} \mathrm{~d} x \mathrm{~d} y \mathrm{~d} z \Delta_{12}(x) \Delta_{12}(y) \Delta_{12}(z) \mathcal{K}(x, y, z) \\
& =-\frac{\pi^{3}}{8} R(\chi)^{3} \sum_{k, l, m=1}^{2} \mathcal{K}\left(\rho_{k}, \rho_{l}, \rho_{m}\right) .
\end{aligned}
$$




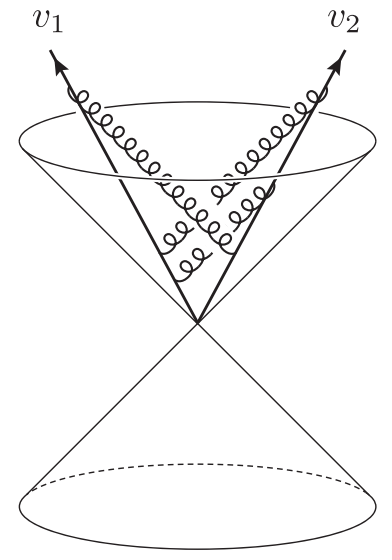

(a)

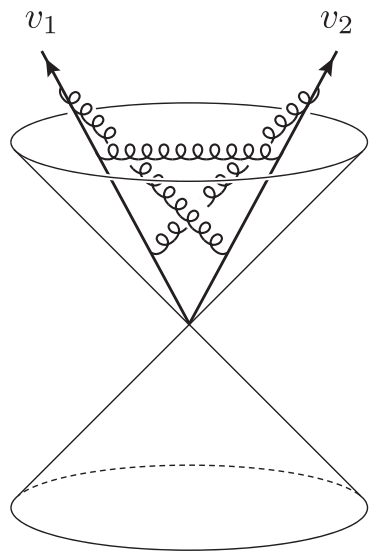

(b)

Figure 10. Triple cut of the two three-loop diagrams that have a single pole divergence. In (a) the non-vanishing triple cut of $F^{(3)}$ is illustrated by the exchange of three lightlike gluons. In contrast, (b) shows graphically that the triple cut of the maximally crossed diagram vanishes, because the three gluons cannot simultaneously be aligned with the light cone (i.e., go on shell).

In the second line we inserted the form of $\Delta_{12}$ given in eq. (3.15) and then integrated out the delta functions. Now observe that $\mathcal{K}\left(\rho_{k}, \rho_{l}, \rho_{m}\right)$, which is implicitly defined in eq. (4.26), is non-zero if and only if $(k, l, m)=(1,2,2)$. Indeed, in the first term of eq. (4.26), the logarithm is non-zero only when $k \neq m$, while the step functions dictate that $\rho_{k} \leq \rho_{m} \leq \rho_{l}$. Since $\rho_{1}<\rho_{2}$, we must therefore have $k=1$ and $l=m=2$. An identical argument applies to the second term of eq. (4.26). We arrive at the simple result

$$
\mathrm{Cut}_{x, y, z} \mathcal{F}^{(3)}=-\frac{\pi^{3}}{2} R(\chi)^{3} \log ^{2} \chi .
$$

This completes the evaluation of the triple-cut contribution to eq. (4.29).

We now turn to the single cuts. We will focus on the contribution $\mathrm{Cut}_{x} \mathcal{F}^{(3)}$, the remaining cuts being computed completely analogously. (Also recall from the discussion below eq. (4.26) that $\mathrm{Cut}_{y} \mathcal{F}^{(3)}=\mathrm{Cut}_{z} \mathcal{F}^{(3)}$, so that only one additional single cut needs to be computed.) Using the partial-fractioned expressions for $\Delta_{12}$ and $P_{12}^{[0]}$ in eq. (3.15) and integrating out the delta function we find, dropping $\pm i \eta$ for notational convenience,

$$
\begin{aligned}
\operatorname{Cut}_{x} \mathcal{F}^{(3)} & =\int_{0}^{1} \mathrm{~d} x \mathrm{PV} \int_{0}^{1} \mathrm{~d} y \mathrm{PV} \int_{0}^{1} \mathrm{~d} z \Delta_{12}(x) P_{12}^{[0]}(y) P_{12}^{[0]}(z) \mathcal{K}(x, y, z) \\
& =-\frac{\pi}{8} R(\chi)^{3} \sum_{k=1}^{2} \mathrm{PV} \int_{\rho_{k}}^{1} \mathrm{~d} y\left(\frac{1}{y-\rho_{1}}-\frac{1}{y-\rho_{2}}\right) \mathrm{PV} \int_{\rho_{k}}^{y} \mathrm{~d} z\left(\frac{1}{z-\rho_{1}}-\frac{1}{z-\rho_{2}}\right) \log ^{2}\left(\frac{1-\rho_{k}}{\rho_{k}} \frac{z}{1-z}\right),
\end{aligned}
$$

exploiting the residual $y \longleftrightarrow z$ symmetry of the integrand.

The principal-value integrals may be evaluated as the corresponding full integrals minus the imaginary part of the latter. Formulas of imaginary parts of multiple polylogarithms are listed up to weight four in appendix A. The first PV integral gives rise to step functions involving the variables $\rho_{1}, \rho_{2}$ and $y$. Splitting the $y$-integral in the $k=1$ term into two 
integrals with the respective domains $\left[\rho_{1}, \rho_{2}\right]$ and $\left[\rho_{2}, 1\right]$ allows all step functions to be resolved. Ultimately, the cut is found to evaluate into the expression

$$
\begin{aligned}
\operatorname{Cut}_{x} \mathcal{F}^{(3)}=\frac{\pi}{2} R(\chi)^{3}[ & -\frac{1}{3} \log ^{4} \chi+\log ^{2} \chi\left(H_{2}\left(\chi^{2}\right)-3 \zeta_{2}\right)-2 \log \chi\left(H_{3}\left(\chi^{2}\right)-\zeta_{3}\right) \\
& \left.+H_{2,2}\left(\chi^{2}\right)+2 H_{4}\left(\chi^{2}\right)-\zeta_{2} H_{2}\left(\chi^{2}\right)-\frac{1}{4} \zeta_{4}\right] .
\end{aligned}
$$

This result is expressed in terms of harmonic polylogarithms, defined through eq. (A.6). Similarly, the $y$ - and $z$-cuts are found to take the form

$$
\begin{aligned}
\operatorname{Cut}_{y} \mathcal{F}^{(3)}=\operatorname{Cut}_{z} \mathcal{F}^{(3)}=\frac{\pi}{4} R(\chi)^{3}[ & -\frac{1}{3} \log ^{4} \chi+\log ^{2} \chi\left(H_{2}\left(\chi^{2}\right)+3 \zeta_{2}\right) \\
& \left.+2 H_{3,1}\left(\chi^{2}\right)+H_{2,2}\left(\chi^{2}\right)-\zeta_{2} H_{2}\left(\chi^{2}\right)+\frac{5}{4} \zeta_{4}\right] .
\end{aligned}
$$

Combining all single and triple cuts according to eq. (4.29) yields the following imaginary part

$$
\begin{aligned}
\operatorname{Im} \mathcal{F}^{(3)}=\pi R(\chi)^{3}[ & -\frac{1}{3} \log ^{4} \chi+\log ^{2} \chi\left(H_{2}\left(\chi^{2}\right)+3 \zeta_{2}\right)-\log \chi\left(H_{3}\left(\chi^{2}\right)-\zeta_{3}\right) \\
& \left.+H_{3,1}\left(\chi^{2}\right)+H_{2,2}\left(\chi^{2}\right)+H_{4}\left(\chi^{2}\right)-\zeta_{2} H_{2}\left(\chi^{2}\right)+\frac{1}{2} \zeta_{4}\right] .
\end{aligned}
$$

As a crosscheck of this result, we can alternatively compute the imaginary part of the three-loop ladder by evaluating the diagram for space-like kinematics $v_{1} \cdot v_{2}<0$, in which case it will be purely real (cf. the discussion at the end of section 3), and subsequently perform the analytic continuation to time-like kinematics.

To the leading order in $\epsilon$, the three-loop ladder is given by eq. (4.28), although we must bear in mind that for space-like kinematics the propagator roots $\rho_{k}$ are given by the lower case of eq. (3.16). Inserting into eq. (4.28) the expressions for $P_{12}^{[0]}$ and $\mathcal{K}(x, y, z)$ given in eqs. (3.15) and (4.26), respectively, the diagram is directly expressible in terms of multiple polylogarithms,

$$
\widetilde{\mathcal{F}}^{(3)}=\frac{R(\chi)^{3}}{4} \sum_{i, j, k, l, m=0,1}(-1)^{i+j+k+l+m} G\left(\rho_{i+1}, \rho_{j+1}, k, l, \rho_{m+1} ; 1\right),
$$

where the tilde on the left-hand side indicates that the diagram is computed for space-like kinematics. We can use the algorithm in appendix $\mathrm{B}$ to recast this representation in terms of polylogarithms with constant indices. In fact, the three-loop ladder diagram can be expressed in terms of harmonic polylogarithms,

$$
\begin{aligned}
\widetilde{\mathcal{F}}^{(3)}=\frac{R(\chi)^{3}}{4}[ & -\frac{4}{15} \log ^{5} \chi+\frac{4}{3} \log ^{3} \chi\left(H_{2}\left(\chi^{2}\right)-\zeta_{2}\right)-2 \log ^{2} \chi\left(H_{3}\left(\chi^{2}\right)-\zeta_{3}\right) \\
& +4 \log \chi\left(H_{3,1}\left(\chi^{2}\right)+H_{2,2}\left(\chi^{2}\right)+H_{4}\left(\chi^{2}\right)+\zeta_{2} H_{2}\left(\chi^{2}\right)+\frac{3}{2} \zeta_{4}\right) \\
& -6 H_{4,1}\left(\chi^{2}\right)-6 H_{3,2}\left(\chi^{2}\right)-4 H_{2,3}\left(\chi^{2}\right)-6 H_{5}\left(\chi^{2}\right)-2 \zeta_{2} H_{3}\left(\chi^{2}\right) \\
& \left.+4 \zeta_{3} H_{2}\left(\chi^{2}\right)+3 \zeta_{5}+2 \zeta_{2} \zeta_{3}\right] .
\end{aligned}
$$


We have cross-checked this expression with previous results in the literature, finding agreement. ${ }^{7}$

We can now find the result for the three-loop ladder diagram in time-like kinematics by performing the analytic continuation $\chi \rightarrow-1 / \chi-i \eta$ on eq. (4.37). Under the analytic continuation, the rational function $R(\chi)$ picks up a minus sign, while polylogarithms transform according to

$$
\begin{aligned}
\log \chi & \rightarrow \log (-1 / \chi-i \eta)=-\log \chi-\pi i \\
H_{\vec{a}}\left(\chi^{2}\right) & \rightarrow H_{\vec{a}}\left(1 / \chi^{2}+i \eta\right)
\end{aligned}
$$

Thus, all harmonic polylogarithms are evaluated slightly above the branch cut $[1, \infty)$. They were subsequently expressed in terms of $H_{\vec{a}}\left(\chi^{2}\right)$ and $\log \chi$ using the Mathematica package HPL $[77,78]$. In this way, we find the following result for the three-loop ladder with time-like kinematics,

$$
\begin{aligned}
\mathcal{F}^{(3)}=-\frac{R(\chi)^{3}}{4}[ & \frac{4}{15} \log ^{5} \chi-\frac{4}{3} \log ^{3} \chi\left(H_{2}\left(\chi^{2}\right)+11 \zeta_{2}\right)+2 \log ^{2} \chi\left(H_{3}\left(\chi^{2}\right)-\zeta_{3}\right) \\
& -4 \log \chi\left(H_{3,1}\left(\chi^{2}\right)+H_{2,2}\left(\chi^{2}\right)+H_{4}\left(\chi^{2}\right)-5 \zeta_{2} H_{2}\left(\chi^{2}\right)-\frac{27}{2} \zeta_{4}\right) \\
& +6 H_{4,1}\left(\chi^{2}\right)+6 H_{3,2}\left(\chi^{2}\right)+4 H_{2,3}\left(\chi^{2}\right)+6 H_{5}\left(\chi^{2}\right)-10 \zeta_{2} H_{3}\left(\chi^{2}\right)-4 \zeta_{3} H_{2}\left(\chi^{2}\right) \\
& -3 \zeta_{5}+10 \zeta_{2} \zeta_{3} \\
& +4 \pi i\left(\frac{1}{3} \log ^{4} \chi-\log ^{2} \chi\left(H_{2}\left(\chi^{2}\right)+3 \zeta_{2}\right)+\log \chi\left(H_{3}\left(\chi^{2}\right)-\zeta_{3}\right)\right. \\
& \left.\left.\quad-H_{3,1}\left(\chi^{2}\right)-H_{2,2}\left(\chi^{2}\right)-H_{4}\left(\chi^{2}\right)+\zeta_{2} H_{2}\left(\chi^{2}\right)-\frac{1}{2} \zeta_{4}\right)\right] .
\end{aligned}
$$

We observe that the imaginary part of eq. (4.39) agrees with the result found in eq. (4.35), as expected. We conclude that the cutting prescription for the three-loop ladder stated in eq. (4.29) produces the correct imaginary part. The cutting prescription (4.29) is illustrated in figure 6.

\subsection{Two-loop web with three Wilson lines}

The formalism of section 3 allows us to compute the imaginary part of eikonal diagrams only to the leading order in $\epsilon$. This appears to limit the applicability of the approach, but in practice a large class of diagrams have only simple poles in $\epsilon$, and the coefficient of the $\frac{1}{\epsilon}$ pole of the correlator of Wilson lines defines physical observables of interest, such as for example the cusp anomalous dimension (i.e., the anomalous dimension of the correlator

\footnotetext{
${ }^{7}$ More specifically, adding the diagram in figure 9 to the maximally-crossed three-loop ladder, which we have computed by the same methods, we find agreement with eq. (A.1) of ref. [28] (in its published version; or alternatively eq. (73) of the corresponding arXiv e-print (v2)). Furthermore, the two color structures of the $(3,3)$ web are linear combinations of these two diagrams, according to eq. (4.26) in ref. [39]. Inserting our results for the two diagrams, we recover the color structures in their eqs. (4.29) and (4.33) (where the basis functions are given explicitly in appendix A), thereby cross-checking our results for both diagrams individually, and in particular our result in eq. (4.37) for the three-loop non-planar ladder.
} 


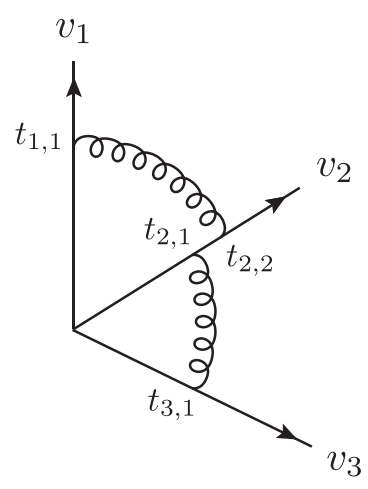

(a)

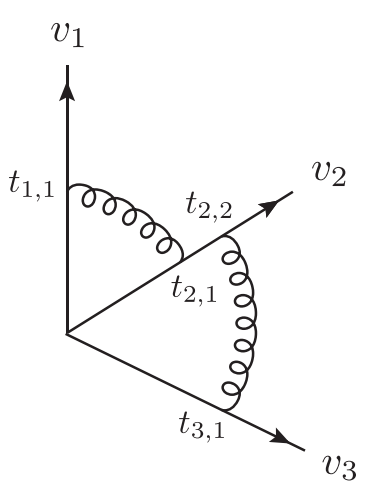

(b)

Figure 11. The two diagrams constituting the $(1,2,1)$ web. The antisymmetric combination of the two diagrams is not captured by exponentiation of the one-loop subdiagrams, and this linear combination of the diagrams, defining the web, appears in the exponent. Each separate diagram diverges as $\mathcal{O}\left(\frac{1}{\epsilon^{2}}\right)$, but the leading divergences conspire to cancel in the web, leaving an $\mathcal{O}\left(\frac{1}{\epsilon}\right)$ divergence.

of two Wilson lines, cf. eq. (2.8)). As it turns out, the cusp anomalous dimension can be expressed entirely in terms of diagrams with simple poles in $\epsilon$ (once the diagrams are expressed in terms of the renormalized coupling $g_{R}$ ). We observed this already for Abelian gauge theories in eq. (2.7), but the statement extends to the case of non-Abelian gauge theories as well. This owes to the non-Abelian exponentiation theorem for two Wilson lines $[4,5]$ which states that the two-line correlator can be written as the exponential of the sum of webs, defined as the subclass of diagrams which are eikonal-line two-particle irreducible. The webs appear in the exponent with their color prefactors appropriately modified to account for the color factors of the complete set of diagrams arising from expanding the exponential.

Over the past four years, non-Abelian exponentiation has been shown to generalize to correlators of an arbitrary number of Wilson lines [8-14]. In this section we will study the interplay of this remarkable theorem with the formalism of section 3 .

The statement of non-Abelian exponentiation in the multi-line case requires a new classification of the set of diagrams which appear in the exponent. In the multi-line case, a web is defined as a collection of diagrams which are mutually related by permutations of the order of gluon attachments, acting on each Wilson line separately. As an example, consider the $(1,2,1)$ web in figure 11 , whose imaginary part we will turn to shortly.

The labels $(1,2,1)$ refer to the number of gluon attachments on each eikonal line, and the two diagrams are related by permutations of the attachment points, where the permutations act on each line separately. The individual diagrams in figure 11 are clearly not eikonal-line 2PI diagrams. However, the contributions of these diagrams are not entirely reproduced by exponentiation of their one-loop subdiagrams. Indeed, the sum of the oneloop subdiagrams spanned by the pairs of lines $\left\{v_{1}, v_{2}\right\}$ and $\left\{v_{2}, v_{3}\right\}$ will appear in the exponent, but upon expanding the exponential to second order, these subdiagrams only reproduce the symmetric linear combination of the two-loop diagrams. To compensate, the 
antisymmetric linear combination of the two-loop diagrams, which defines the web, must be added to the exponent as a contribution. Denoting the color and kinematical factors of the diagrams in figure 11 as $C_{\mathrm{a}, \mathrm{b}}$ and $\mathcal{F}_{\mathrm{a}, \mathrm{b}}$, the web is accordingly defined as

$$
W_{(1,2,1)}^{(2)}=\left(C_{\mathrm{a}}, C_{\mathrm{b}}\right)\left(\begin{array}{cc}
\frac{1}{2} & -\frac{1}{2} \\
-\frac{1}{2} & \frac{1}{2}
\end{array}\right)\left(\begin{array}{c}
\mathcal{F}_{\mathrm{a}} \\
\mathcal{F}_{\mathrm{b}}
\end{array}\right)=\frac{1}{2}\left(C_{\mathrm{a}}-C_{\mathrm{b}}\right)\left(\mathcal{F}_{\mathrm{a}}-\mathcal{F}_{\mathrm{b}}\right) .
$$

We can now state the general non-Abelian exponentiation theorem [8-14]. Recalling the definition in eq. (1.1) of the Wilson line $\Phi_{v}$ spanned by the four-velocity $v^{\mu}$, the statement is that the correlator of an arbitrary number of Wilson lines is given as the exponential of the sum of webs,

$$
\left\langle\Phi_{v_{1}} \cdots \Phi_{v_{n}}\right\rangle=\exp \left(\sum_{i \in\{\text { webs }\}} C_{i}^{T} R_{i} \mathcal{F}_{i}\right),
$$

where each web $i$ contributes to the exponent through the color $C_{i}$ and kinematical $\mathcal{F}_{i}$ factors of its constituent diagrams, weighted by means of the web mixing matrix $R_{i}$ in analogy with eq. (4.40). The web mixing matrices can be computed systematically by means of the replica trick of statistical mechanics [8, 79]. Among several properties they satisfy the zero-sum-row condition $\sum_{b} R_{a b}=0$ which ensures that the symmetric linear combination of the constituent diagrams is projected out [10].

The mixing matrices satisfy an additional weighted zero-sum column condition which ensures that the leading divergence of the constituent diagrams of a web conspire to cancel when the diagrams are added. This is a general feature of webs, ultimately following from their renormalization properties $[8,9,11]$, leaving in many cases a web with an $\mathcal{O}\left(\frac{1}{\epsilon}\right)$ divergence. As a result, webs are particularly amenable to the formalism of section 3 . It is here important to keep in mind that the cutting prescription should be applied to an entire web rather than its constituent diagrams separately, as the separate imaginary parts, computed to leading order in $\epsilon$, will cancel.

To illustrate the procedure in detail, we turn to the web in figure 11 and compute its imaginary part. In analogy with sections 4.1 and 4.2 , we take $\cosh \gamma_{12} \equiv v_{1} \cdot v_{2}>0$ and $\cosh \gamma_{23} \equiv v_{2} \cdot v_{3}>0$, in order to have a non-vanishing contribution to the imaginary part from both kinematical channels, and set $\chi \equiv e^{-\gamma_{12}}$ and $\psi \equiv e^{-\gamma_{23}}$. Our first task will be to show that the leading $\mathcal{O}\left(\frac{1}{\epsilon^{2}}\right)$ divergences of the individual diagrams conspire to cancel, leaving an $\mathcal{O}\left(\frac{1}{\epsilon}\right)$ divergence. We will then apply the cutting prescription of eqs. (3.10)-(3.12) directly to the web written in a form with a manifest $\mathcal{O}\left(\frac{1}{\epsilon}\right)$ divergence.

Let us start by considering diagram $6 \mathrm{a}$ in figure 11. Its kinematical factor is given by

$$
\mathcal{F}_{\mathrm{a}}=C^{(2)} \mu^{4 \epsilon} \int_{0}^{\infty} \frac{\mathrm{d} t_{1,1} \mathrm{~d} t_{2,1} \mathrm{~d} t_{2,2} \mathrm{~d} t_{3,1} \theta\left(t_{2,2}-t_{2,1}\right)\left(v_{1} \cdot v_{2}\right)\left(v_{2} \cdot v_{3}\right)}{\left[-\left(t_{1,1} v_{1}-t_{2,2} v_{2}\right)^{2}+i \eta\right]^{1-\epsilon}\left[-\left(t_{2,1} v_{2}-t_{3,1} v_{3}\right)^{2}+i \eta\right]^{1-\epsilon}},
$$

where $C^{(2)}$ contains coupling constants etc., but no color factor. In analogy with sections 4.1 and 4.2 our first task is to write this expression in the form of eq. (3.7). This is achieved through the changes of variables in eq. (3.2) (with $\left.t_{\ell_{j}, m_{j}}=t_{2,3-j}\right)$, setting $\left(x_{1}, x_{2}\right)=(x, y)$ for convenience. 
After these transformations, the kinematical factor takes the form

$$
\mathcal{F}_{\mathrm{a}}=C^{(2)} \int_{0}^{1} \mathrm{~d} x \mathrm{~d} y P_{12}^{[\epsilon]}(x) P_{23}^{[\epsilon]}(y) K(x, y),
$$

where the kernel is given by

$$
\begin{aligned}
K(x, y) & =\mu^{4 \epsilon} \int_{0}^{\infty} \frac{\mathrm{d} \rho_{1} \mathrm{~d} \rho_{2}}{\left(\rho_{1} \rho_{2}\right)^{1-2 \epsilon}} \theta\left(\rho_{1} x-\rho_{2} y\right) \\
& =\frac{\Gamma(4 \epsilon)}{2 \epsilon}\left(\frac{\mu}{\Lambda}\right)^{4 \epsilon}\left[u^{2 \epsilon}{ }_{2} F_{1}(2 \epsilon, 4 \epsilon ; 1+2 \epsilon ;-u)\right]_{u=y / x}^{u=\infty} .
\end{aligned}
$$

The result for $K(x, y)$ was obtained by applying the substitution in eq. (3.6) and performing the remaining integrations in complete analogy with section 4.1. The second diagram of the web, $\mathcal{F}_{\mathbf{b}}$, differs only in the step function which reads $\theta\left(\rho_{2} y-\rho_{1} x\right)$, changing the lower integration bound in eq. (4.44) from $y / x$ to $x / y$. After expanding the gamma function and the hypergeometric function in $\epsilon$, we thus find the kinematical factor of the $(1,2,1)$ web to take the form

$$
\mathcal{F}_{\mathrm{a}}-\mathcal{F}_{\mathrm{b}}=\frac{C^{(2)}}{8 \epsilon^{2}}\left(\frac{\mu}{\Lambda}\right)^{4 \epsilon} \int_{0}^{1} \mathrm{~d} x \mathrm{~d} y P_{12}^{[\epsilon]}(x) P_{23}^{[\epsilon]}(y)\left[\left(\frac{x}{y}\right)^{2 \epsilon}-\left(\frac{y}{x}\right)^{2 \epsilon}\right]\left(1+\mathcal{O}\left(\epsilon^{2}\right)\right) .
$$

Upon expanding $[\cdots]$ in $\epsilon$, we observe that the leading poles of the separate diagrams cancel, leaving an $\mathcal{O}\left(\frac{1}{\epsilon}\right)$ divergence, in agreement with the discussion above.

Factoring out the remaining pole, we can write the web in the convenient form

$$
W_{(1,2,1)}^{(2)}=\frac{C_{\mathrm{a}}-C_{\mathrm{b}}}{2} \frac{C^{(2)}}{2 \epsilon}\left(\frac{\mu}{\Lambda}\right)^{4 \epsilon} \mathcal{F}_{(1,2,1)}^{(2)},
$$

where $\mathcal{F}_{(1,2,1)}^{(2)}$ is finite and given to the leading order in $\epsilon$ by

$$
\mathcal{F}_{(1,2,1)}^{(2)}=\int_{0}^{1} \mathrm{~d} x \mathrm{~d} y P_{12}^{[0]}(x) P_{23}^{[0]}(y) \log \frac{x}{y} .
$$

As the prefactor of $\mathcal{F}_{(1,2,1)}^{(2)}$ in eq. (4.46) is real, it factors out on both sides of eq. (3.12), yielding the formula

$$
\operatorname{Im} \mathcal{F}_{(1,2,1)}^{(2)}=\operatorname{Cut}_{x} \mathcal{F}_{(1,2,1)}^{(2)}+\operatorname{Cut}_{y} \mathcal{F}_{(1,2,1)}^{(2)}
$$

More explicitly, by inserting the definition of the operator $\mathrm{Cut}_{x_{i}}$ in eq. (3.11), we have

$$
\operatorname{Im} \mathcal{F}_{(1,2,1)}^{(2)}=\int_{0}^{1} \mathrm{~d} x \operatorname{PV} \int_{0}^{1} \mathrm{~d} y \Delta_{12}(x) P_{23}^{[0]}(y) \log \frac{x}{y}+\mathrm{PV} \int_{0}^{1} \mathrm{~d} x \int_{0}^{1} \mathrm{~d} y P_{12}^{[0]}(x) \Delta_{23}(y) \log \frac{x}{y}
$$

We observe that the second term equals minus the first term with the two cusp angles and integration variables interchanged, making the imaginary part of the web antisymmetric under the interchange $\gamma_{12} \longleftrightarrow \gamma_{23}$. (The antisymmetry is of course inherited from the 
full web which has this property by construction.) This observation allows us to write the imaginary part in the manifestly antisymmetric form

$$
\operatorname{Im} \mathcal{F}_{(1,2,1)}^{(2)}=J(\chi, \psi)-J(\psi, \chi),
$$

where the auxiliary function is defined as the first term of eq. (4.49),

$$
J(\chi, \psi)=\int_{0}^{1} \mathrm{~d} x \operatorname{PV} \int_{0}^{1} \mathrm{~d} y \Delta_{12}(x) P_{23}^{[0]}(y) \log \frac{x}{y} .
$$

Evaluation of the imaginary part of the web thus reduces to the evaluation of the integral $J(\chi, \psi)$. The latter can be computed by recalling the partial-fractioned expressions for $\Delta_{12}$ and $P_{23}^{[0]}$ given in eq. (3.15) which in the present notation read

$$
\begin{aligned}
& \Delta_{12}(x)=-\pi \frac{R(\chi)}{2}\left(\delta\left(x-\rho_{1}(\chi)\right)+\delta\left(x-\rho_{2}(\chi)\right)\right), \\
& P_{23}^{[0]}(y)=\frac{R(\psi)}{2}\left(\frac{1}{y-\rho_{1}(\psi)+i \eta}-\frac{1}{y-\rho_{2}(\psi)-i \eta}\right),
\end{aligned}
$$

where we wrote out the expressions explicitly to emphasize their dependence on the two distinct kinematical invariants $\chi$ and $\psi$.

Integrating out the delta functions in eq. (4.51) leaves one principal-value integral to be evaluated. This integral is computed as the corresponding full integral minus its imaginary part, as explained in section $4.1 .^{8}$ In this way we find

$$
\begin{gathered}
J(\chi, \psi)=-\frac{\pi}{4} R(\chi) R(\psi) \sum_{k=1}^{2} \mathrm{PV} \int_{0}^{1} \mathrm{~d} y\left(\frac{1}{y-\rho_{1}(\psi)+i \eta}-\frac{1}{y-\rho_{2}(\psi)-i \eta}\right) \log \left(\frac{\rho_{k}(\chi)}{y}\right) \\
=-\frac{\pi}{4} R(\chi) R(\psi)\left(-4 \operatorname{Li}_{2}(-\psi)+\log ^{2} \psi-4 \log \psi(\log (\psi+1)-\log (\chi+1))\right. \\
\left.-2 \log \chi \log \psi-2 \zeta_{2}\right) .
\end{gathered}
$$

Upon the antisymmetrization in eq. (4.50) the terms on the last line of eq. (4.53) cancel, and we find the following result for the imaginary part of the $(1,2,1)$ web,

$$
\begin{aligned}
\operatorname{Im} \mathcal{F}_{(1,2,1)}^{(2)}=-\pi R(\chi) R(\psi)( & \operatorname{Li}_{2}(-\chi)-\operatorname{Li}_{2}(-\psi)-\frac{1}{4}\left(\log ^{2} \chi-\log ^{2} \psi\right) \\
& +(\log \chi+\log \psi)(\log (\chi+1)-\log (\psi+1))) .
\end{aligned}
$$

As a crosscheck of this result, we can alternatively compute the imaginary part of the web by evaluating the diagram for space-like kinematics $v_{1} \cdot v_{2}<0$ and $v_{2} \cdot v_{3}<0$, in which case it will be purely real (cf. the discussion at the end of section 3 ), and subsequently perform the analytic continuation to time-like kinematics.

To the leading order in $\epsilon$, the web is given by eq. (4.47), although we must bear in mind that for space-like kinematics the propagator roots $\rho_{k}$ are given by the lower case of

\footnotetext{
${ }^{8}$ To proceed we assume, without loss of generality, that $\psi<\chi$. This fixes $\rho_{1}(\chi)<\rho_{1}(\psi)<\rho_{2}(\psi)<\rho_{2}(\chi)$, which allows the step functions in eqs. (A.22)-(A.25) to be resolved.
} 
eq. (3.16). We now insert into eq. (4.47) the expressions for $P_{12}^{[0]}$ given in eq. (3.15) and perform the integrals in analogy with the calculations in eqs. (4.19)-(4.20) for the case of the non-planar two-loop ladder. This leads to the following result for the web in space-like kinematics,

$$
\widetilde{\mathcal{F}}_{(1,2,1)}^{(2)}=R(\chi) R(\psi)(L(\psi) \log \chi-L(\chi) \log \psi),
$$

where we introduced the auxiliary function

$$
L(\chi)=-\operatorname{Li}_{2}(1-\chi)-\frac{1}{4} \log ^{2} \chi .
$$

These expressions are consistent with results previously obtained in the literature, see for example eq. (3.11) in ref. [38], as well as references therein. We can now obtain the result for the web in time-like kinematics by performing the analytic continuations $\chi \rightarrow-1 / \chi-i \eta$ and $\psi \rightarrow-1 / \psi-i \eta$ on eq. (4.55). Under the analytic continuation, the functions appearing in eq. (4.55) transform as

$$
\begin{aligned}
R(z) \rightarrow & -R(z) \\
\log z \rightarrow & -\log z-\pi i \\
L(z) \rightarrow & -\operatorname{Li}_{2}(-z)-\log z \log (z+1)+\frac{1}{4} \log ^{2} z-\frac{1}{2} \zeta_{2} \\
& \quad-\pi i\left(\log (z+1)-\frac{1}{2} \log z\right) .
\end{aligned}
$$

Upon analytic continuation in $\chi$ and $\psi$ we thus find the following result for the web with time-like kinematics,

$$
\begin{aligned}
\mathcal{F}_{(1,2,1)}^{(2)}=R(\chi) R(\psi)[ & -i \pi\left(\operatorname{Li}_{2}(-\chi)-\frac{1}{4} \log ^{2} \chi+(\log \chi+\log \psi) \log (\chi+1)\right) \\
& +\log \chi \operatorname{Li}_{2}(-\psi)-\left(\log \chi \log \psi-6 \zeta_{2}\right) \log (\chi+1) \\
& \left.+\frac{1}{4}\left(\log \chi \log \psi-10 \zeta_{2}\right) \log \chi\right]-(\chi \longleftrightarrow \psi)
\end{aligned}
$$

We observe that the imaginary part of eq. (4.58) agrees with the result found in eq. (4.54), as expected. We conclude that the cutting prescription for the two-loop web stated in eq. (4.48) produces the correct imaginary part. The graphical representation of the cutting prescription (4.48) is similar to that in figure 8 , and we omit it here.

In the above we have computed the imaginary part of the web with time-like kinematics. As the web depends on two distinct angles, we may also consider the diagram in the case of mixed time- and space-like kinematics, for example $\cosh \gamma_{12} \equiv v_{1} \cdot v_{2}>0$ and $\cosh \gamma_{23} \equiv-v_{2} \cdot v_{3}>0 .{ }^{9}$ A natural question is then whether also in this case the imaginary part is computed correctly by the formalism of section 3. As we shall see shortly, the formalism readily applies, with the one difference that the imaginary part has no contribution from the $\psi$-channel, as propagators stretched between mutually space-like eikonal lines

\footnotetext{
${ }^{9}$ The opposite-type kinematics $v_{1} \cdot v_{2}<0$ and $v_{2} \cdot v_{3}>0$ is of course equivalent by the antisymmetry of the web under the interchange $\gamma_{12} \longleftrightarrow \gamma_{23}$ of the cusp angles.
} 
have vanishing cuts. Put differently, the discontinuity in the $\psi$-channel does not contribute to the imaginary part, cf. eq. (3.14).

Returning to the formula for the imaginary part in the explicit form (4.49), we observe that in the above case of mixed time- and space-like kinematics, the roots of the propagator $P_{23}^{[0]}(y)$ lie outside the range of integration, cf. the remarks below eq. (3.16). As a result, the second term in eq. (4.49) vanishes and in the first term the principal-value prescription may be dropped,

$$
\operatorname{Im} \mathcal{F}_{(1,2,1)}^{(2)}=\int_{0}^{1} \mathrm{~d} x \mathrm{~d} y \Delta_{12}(x) P_{23}^{[0]}(y) \log \frac{x}{y} .
$$

After inserting eq. (4.52) and integrating out the delta functions, the imaginary part is readily expressed in terms of multiple polylogarithms; these in turn can be simplified into classical polylogarithms, yielding

$$
\begin{aligned}
\operatorname{Im} \mathcal{F}_{(1,2,1)}^{(2)}=\frac{\pi}{4} R(\chi) R(\psi) & \left(-2 G\left(\rho_{2}(\psi), 0 ; 1\right)+2 G\left(\rho_{1}(\psi), 0 ; 1\right)\right. \\
& +G\left(0 ; \rho_{2}(\chi)\right) G\left(\rho_{2}(\psi) ; 1\right)+G\left(0 ; \rho_{1}(\chi)\right) G\left(\rho_{2}(\psi) ; 1\right) \\
& \left.-G\left(0 ; \rho_{2}(\chi)\right) G\left(\rho_{1}(\psi) ; 1\right)-G\left(0 ; \rho_{1}(\chi)\right) G\left(\rho_{1}(\psi) ; 1\right)\right) \\
=\pi R(\chi) R(\psi)( & \left.\operatorname{Li}_{2}(\psi)-\log \psi\left(\frac{1}{4} \log \psi-\log (1-\psi)+\log (\chi+1)-\frac{1}{2} \log \chi\right)-\zeta_{2}\right) .
\end{aligned}
$$

Using eq. (4.57) it is straightforward to verify that this result agrees with the imaginary part acquired by eq. (4.55) upon the analytic continuation $\chi \rightarrow-1 / \chi-i \eta$. We conclude that the formula (3.12) reproduces the correct imaginary part of the web also for mixed time- and space-like kinematics, as expected.

\section{Position-space cuts of eikonal diagrams with internal vertices}

In this section we turn to the application of the formalism of ref. [1] to diagrams with internal (i.e., three- and four-gluon) vertices. Here we provide details on the calculation of the imaginary part of the diagram involving a three-gluon vertex connected to three Wilson lines, as illustrated in figure 12.

The integrated result for the diagram in figure 12 was first obtained in refs. $[34,35]$ using a Mellin-Barnes representation of the two loop-momentum integrals. In terms of the cusp angles $\gamma_{i j}$, defined through $\cosh \gamma_{i j}=-v_{i} \cdot v_{j}$, it is given by

$$
\widetilde{F}_{3 g}=-i f^{a b c} \mathbf{T}_{1}^{a} \mathbf{T}_{2}^{b} \mathbf{T}_{3}^{c} \frac{2}{\epsilon}\left(\frac{\alpha_{s}}{4 \pi}\right)^{2} \sum_{i, j, k=1}^{3} \varepsilon_{i j k} \gamma_{i j}^{2} \gamma_{k i} \operatorname{coth} \gamma_{k i}
$$

This expression is valid for an unphysical configuration with space-like kinematics for all pairs of Wilson lines, i.e. $v_{i} \cdot v_{j}<0$, as indicated by the tilde on $\widetilde{F}_{3 g}$. In agreement with our observations in section $2, \widetilde{F}_{3 g}$ has no imaginary part. In contrast, in a physical configuration of massive Wilson lines, each velocity is constrained to the unit three-hyperboloid, either 


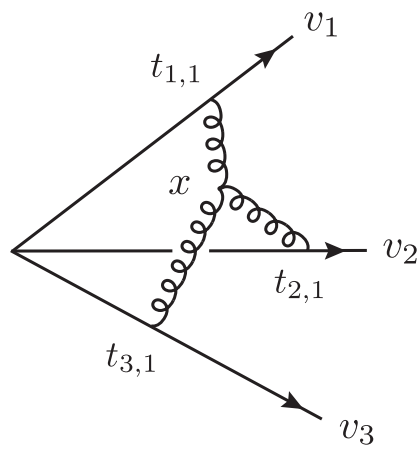

Figure 12. The non-planar two-loop three-gluon vertex diagram.

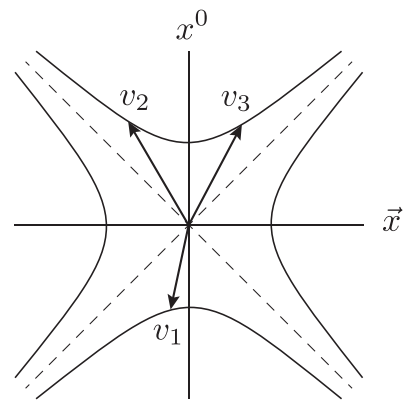

(a)

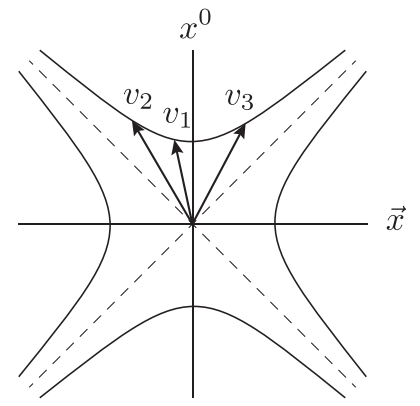

(b)

Figure 13. Physical configurations for three distinct Wilson-line velocities: (a) one incoming and two outgoing lines, or (b) three outgoing lines. Configurations related by time-reversal are omitted. In this section we compute the imaginary part of the three-gluon vertex diagram in the physical configuration (b).

inside the future light cone or inside the past light cone. There are two inequivalent physical configurations, shown in figure 13. In both configurations at least one pair of Wilson lines is time-like separated, i.e. $v_{i} \cdot v_{j}>0$, leading to a non-vanishing imaginary part.

In the following we consider the configuration in figure 13(b), where all Wilson lines correspond to outgoing states, such that $v_{i} \cdot v_{j}>0$ for each pair of Wilson lines. The analytic result for such time-like kinematics, denoted by $F_{3 g}$, is obtained from the spacelike expression $\widetilde{F}_{3 g}$ in eq. (5.1) by analytic continuation $\gamma_{i j} \rightarrow i \pi-\gamma_{i j}$ for all $i \neq j$ (cf. eq. (3.18)). The imaginary part of the resulting expression is

$$
\operatorname{Im} F_{3 g}=-f^{a b c} \mathbf{T}_{1}^{a} \mathbf{T}_{2}^{b} \mathbf{T}_{3}^{c}\left(\frac{\alpha_{s}}{4 \pi}\right)^{2} \frac{2}{\epsilon} \sum_{i, j, k=1}^{3} \varepsilon_{i j k}\left(\gamma_{i j}^{2} \gamma_{k i}-2 \pi^{2} \gamma_{i j}\right) \operatorname{coth} \gamma_{k i} .
$$

In the remainder of this section our task is to compute this imaginary part with our formalism. As in section 3 we first need to extract the leading divergence of $F_{3 g}$ in its position-space representation. The three-gluon vertex diagram has a only a simple pole in $\epsilon$. This divergence is extracted from the radial integral over the three-gluon vertex position. Having extracted the leading divergence, the diagram can be written as $\frac{1}{\epsilon} \times$ (finite). We 
then apply position-space cuts to the finite function. The one-dimensional integrals along the Wilson lines are then trivially performed using the delta functions arising from the cut. The remaining integrations over the direction of the three-gluon vertex are performed numerically, after which the final result is compared to the analytic expression in eq. (5.2).

We start by writing down the position-space representation of $F_{3 g}$. It reads ${ }^{10}$

$$
F_{3 g}=-f^{a b c} \mathbf{T}_{1}^{a} \mathbf{T}_{2}^{b} \mathbf{T}_{3}^{c}\left(\frac{\alpha_{s}}{4 \pi}\right)^{2} \frac{4}{\pi^{2}} \mu^{4 \epsilon} \int \frac{\mathrm{d}^{D} x}{r^{4-6 \epsilon}} \sum_{i, j, k=1}^{3} \varepsilon_{i j k} v_{i} \cdot v_{j} \zeta_{i} \zeta_{k}\left(\frac{\partial}{\partial \zeta_{i}} g\left(\zeta_{i}, \epsilon\right)\right) g\left(\zeta_{j}, \epsilon\right) g\left(\zeta_{k}, \epsilon\right) .
$$

Here the three-gluon vertex position $x$ is integrated over all of Minkowski space. In the integrand $x$ is decomposed into a radial distance $r$ and direction $u$, via $x^{\mu}=r u^{\mu}$, such that $u^{2}=1$ for time-like $x$ and $u^{2}=-1$ for space-like $x$. Dot products between $u$ and the Wilson line velocities are denoted by $\zeta_{i}=v_{i} \cdot u$. The one-dimensional integrals along the Wilson lines are contained in the functions $g\left(\zeta_{i}, \epsilon\right)$, which are defined as

$$
g\left(\zeta_{i}, \epsilon\right)=\int_{0}^{\infty} \frac{\mathrm{d} x_{i}}{\left[-\left(u^{2}-2 x_{i} \zeta_{i}+x_{i}^{2}\right)+i \eta\right]^{1-\epsilon}} .
$$

After a change of variables to hyperspherical coordinates in eq. (5.3), the radial integral contains the overall divergence ${ }^{11}$ and may easily be performed, yielding a factor of $\frac{1}{4 \epsilon}$. Restricting attention to the leading order in $\epsilon$ allows us to set $\epsilon=0$ in the finite function $\mathcal{F}_{3 g}$, yielding

$$
\begin{aligned}
F_{3 g} & =-f^{a b c} \mathbf{T}_{1}^{a} \mathbf{T}_{2}^{b} \mathbf{T}_{3}^{c}\left(\frac{\alpha_{s}}{4 \pi}\right)^{2} \frac{1}{\pi^{2} \epsilon} \mathcal{F}_{3 g}, \\
\mathcal{F}_{3 g} & =\int_{\widetilde{\mathbb{R}}^{1,2}} \mathrm{~d}^{3} u \sum_{i, j, k=1}^{3} \varepsilon_{i j k} v_{i} \cdot v_{j} \zeta_{i} \zeta_{k}\left(\frac{\partial}{\partial \zeta_{i}} g\left(\zeta_{i}, 0\right)\right) g\left(\zeta_{j}, 0\right) g\left(\zeta_{k}, 0\right) .
\end{aligned}
$$

The integration domain for the three-gluon vertex direction $u$ is $\widetilde{\mathbb{R P}}^{1,2} \equiv \mathrm{H}_{+}^{3} \cup \mathrm{H}_{-}^{3} \cup \mathrm{dS}_{3}^{+} \cup$ $\mathrm{dS}_{3}^{-}$, the union of the upper and lower sheets of the unit three-hyperboloid and threedimensional de Sitter space, defined by

$$
\begin{aligned}
\mathrm{H}_{ \pm}^{3} & =\left\{u \in \mathbb{R}^{1,3}: u^{2}=1 \text { and } u_{0} \gtrless 0\right\}, \\
\mathrm{dS}_{3}^{ \pm} & =\left\{u \in \mathbb{R}^{1,3}: u^{2}=-1 \text { and } u_{0} \gtrless 0\right\} .
\end{aligned}
$$

Having written the three-gluon vertex diagram in the form in eq. (5.5), we are ready to apply our formalism to obtain the imaginary part of $\mathcal{F}_{3 g}$ from its cuts. This in turn gives the imaginary part of the full diagram $F_{3 g}$, as they are proportional up to a real constant.

The imaginary part of $\mathcal{F}_{3 g}$ is computed from the formula in eq. (3.12), as illustrated schematically in figure 14 . The cut propagators stretching between the three-gluon vertex and the Wilson lines take the obvious form $\Delta_{i}\left(x_{i}\right)=-\pi \delta\left(u^{2}-2 x_{i} \zeta_{i}+x_{i}^{2}\right)$, rather than

\footnotetext{
${ }^{10}$ See ref. [80] for the corresponding position-space representation of this diagram in Euclidean space.

${ }^{11}$ This divergence is regulated by including an exponential damping factor in the radial integral (cf. eq. (2.3)).
} 


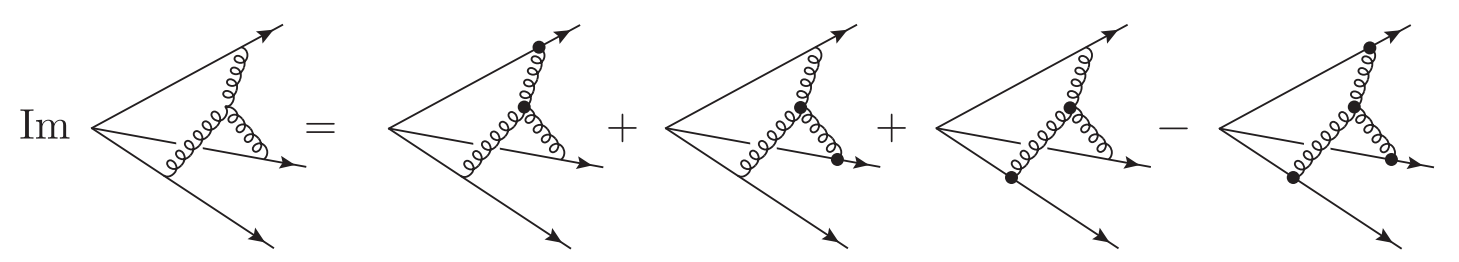

Figure 14. Graphical representation of position-space cuts applied to a diagram with an internal vertex.

eq. (3.10) for propagators connecting two Wilson lines. In order to resolve the support of these delta functions in the different subregions of $\widetilde{R P}^{1,2}$ it is convenient to introduce variables $y_{i}$ that are equal to $\zeta_{i}$, possibly up to a sign depending on the location of $u$. Explicitly, we let $y_{i}=\zeta_{i}$ for $u \in \mathrm{H}_{+}^{3} \cup \mathrm{dS}_{3}^{+}$and $y_{i}=-\zeta_{i}$ for $u \in \mathrm{H}_{-}^{3} \cup \mathrm{dS}_{3}^{-}$. In this way $\zeta_{i}$ flips sign between the \pm regions, but $y_{i}$ does not. In terms of these variables a cut operator acting on a function $g\left(\zeta_{i}, 0\right)$ yields

$$
\operatorname{Cut}_{x_{i}} g\left(\zeta_{i}, 0\right)=-\pi \int_{0}^{\infty} \mathrm{d} x_{i} \delta\left(u^{2}-2 x_{i} \zeta_{i}+x_{i}^{2}\right)= \begin{cases}-\frac{\pi}{2}(1 \pm 1)\left(y_{i}^{2}-1\right)^{-1 / 2} & \text { in } \mathrm{H}_{ \pm}^{3} \\ -\frac{\pi}{2}\left(y_{i}^{2}+1\right)^{-1 / 2} & \text { in } \mathrm{dS}_{3}^{ \pm}\end{cases}
$$

while the principal-value part, due to $\operatorname{Cut}_{x_{j}} g\left(\zeta_{i}, 0\right)$ with $i \neq j$, evaluates to

$$
\mathrm{PV} \int_{0}^{\infty} \frac{\mathrm{d} x_{i}}{-\left(u^{2}-2 x_{i} \zeta_{i}+x_{i}^{2}\right)+i \eta}= \begin{cases} \pm \operatorname{arcosh}\left(y_{i}\right)\left(y_{i}^{2}-1\right)^{-1 / 2} & \text { in } \mathrm{H}_{ \pm}^{3} \\ \mp \operatorname{arsinh}\left(y_{i}\right)\left(y_{i}^{2}+1\right)^{-1 / 2} & \text { in } \mathrm{dS}_{3}^{ \pm}\end{cases}
$$

The right-hand side of eq. (5.7) shows that the cut vanishes for $u$ in the region $\mathrm{H}_{-}^{3}$. This is a consequence of the delta function having no support inside the domain of integration $[0, \infty)$. In the regions $\mathrm{dS}_{3}^{ \pm}$and $\mathrm{H}_{+}^{3}$ there are respectively one and two solutions to the deltafunction constraint, as can also be understood by inspection of figure 15. Focusing on the contributions to the imaginary part from $\mathrm{dS}_{3}^{+}$and $\mathrm{dS}_{3}^{-}$, we see that both the single and the triple cuts acting on the product $g\left(\zeta_{i}, 0\right) g\left(\zeta_{j}, 0\right) g\left(\zeta_{k}, 0\right)$ in $\mathcal{F}_{3 g}$ yield the same results in both regions. But apart from this product of $g$ 's, the sum in eq. (5.5) also contains $\zeta_{i} \zeta_{k} \frac{\partial}{\partial \zeta_{i}}$, which differs by a sign between the two regions. As a result, the imaginary part arising from the regions $\mathrm{dS}_{3}^{+}$and $\mathrm{dS}_{3}^{-}$cancel each other. The upshot is thus that the imaginary part of $\mathcal{F}_{3 g}$ arises solely from the region $\mathrm{H}_{+}^{3}$.

The final step in the computation of $\operatorname{Im} \mathcal{F}_{3 g}$ is now to perform the integration over $u$, the direction of the three-gluon vertex. We do not have analytic results for the integrals involved, but a numerical evaluation is sufficient to show agreement with the analytic formula in eq. (5.2). Let us give a few details regarding the setup of the numerical integration.

The three-gluon vertex direction $u$ may be parametrized explicitly in terms of Minkowski angles $\psi, \vartheta$ and $\phi$. As discussed above, the imaginary part arises solely from the 


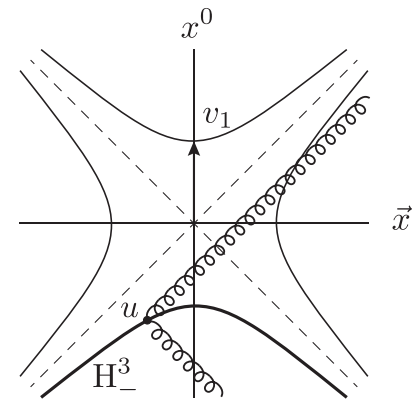

(a)

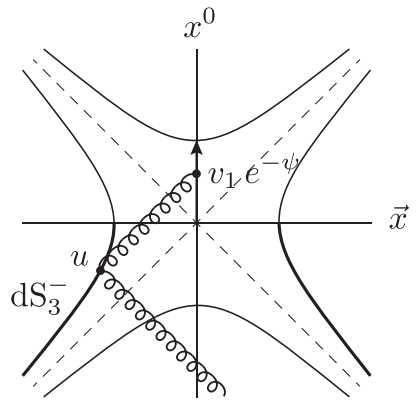

(b)

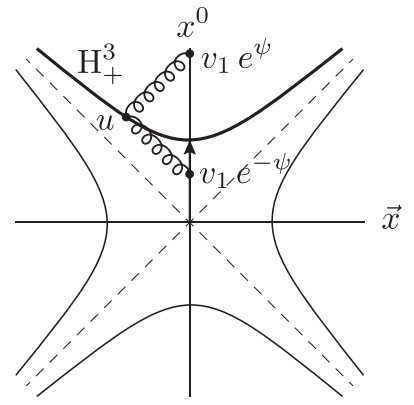

(c)

Figure 15. Spacetime pictures of a Wilson line along the positive $x^{0}$-axis (with normalized velocity $v_{1}$ ) and a three-gluon vertex $u$, located in the region (a) $\mathrm{H}_{-}^{3}$, (b) $\mathrm{dS}_{3}^{-}$or (c) $\mathrm{H}_{+}^{3}$. The region $\mathrm{dS}_{3}^{+}$is very similar to $\mathrm{dS}_{3}^{-}$and is omitted. Each figure shows two lightlike gluons emanating from $u$ : one along the future light cone and the other along the past light cone. Of these two on-shell gluons respectively zero, one or two gluons are able to connect to the Wilson line. In other words, in case (a), (b) and (c) there are respectively zero, one and two solutions to the delta functions coming from the cut operators. This means that in the region $\mathrm{H}_{-}^{3}$ the operator $\mathrm{Cut}_{x_{1}}$ vanishes, thereby producing no imaginary part. Furthermore, as discussed in the main text, the imaginary parts from the space-like regions $\mathrm{dS}_{3}^{+}$and $\mathrm{dS}_{3}^{-}$cancel each other. The only contribution to the imaginary part of the diagram thus arises from the region $\mathrm{H}_{+}^{3}$.

region $\mathrm{H}_{+}^{3}$, which may be parametrized as

$$
\mathrm{H}_{+}^{3}: \begin{cases}u^{0}=\cosh \psi & 0 \leq \psi<\infty \\ u^{1}=\sinh \psi \sin \vartheta \cos \phi & 0 \leq \vartheta \leq \pi \\ u^{2}=\sinh \psi \sin \vartheta \sin \phi & 0 \leq \phi \leq 2 \pi . \\ u^{3}=\sinh \psi \cos \vartheta, & \end{cases}
$$

To facilitate the numerical integration over $\psi \in[0, \infty)$ we perform a further change of variables $z=\tanh \psi$, which has the effect of producing a finite integration domain $z \in[0,1]$. Explicit expressions for $\zeta_{i}=y_{i}=u \cdot v_{i}$ in terms of $z$, the angles $\vartheta, \phi$ and the cusp angles $\gamma_{i j}$ are obtained by choosing a convenient Lorentz frame. For example,

$$
\begin{aligned}
v_{1}^{\mu} & =(1,0,0,0), \\
v_{2}^{\mu} & =\left(\cosh \gamma_{12}, 0,0, \sinh \gamma_{12}\right), \\
v_{3}^{\mu} & =\left(\cosh \gamma_{13}, 0, \sin \theta_{3} \sinh \gamma_{13}, \cos \theta_{3} \sinh \gamma_{13}\right) .
\end{aligned}
$$

These velocities manifestly satisfy $v_{i}^{2}=1$ and $v_{1} \cdot v_{k}=\cosh \gamma_{1 j}$ for $j=2,3$. The remaining identity, $v_{2} \cdot v_{3}=\cosh \gamma_{23}$, fixes $\theta_{3}$ in terms of the cusp angles,

$$
\cos \theta_{3}=\frac{\cosh \gamma_{12} \cosh \gamma_{13}-\cosh \gamma_{23}}{\sinh \gamma_{12} \sinh \gamma_{13}}
$$

The explicit parametrization of the Wilson-line velocities in eq. (5.10) breaks the antisymmetry of $\mathcal{F}_{3 g}$ under interchange of any pair of cusp angles at the integrand level. However, 

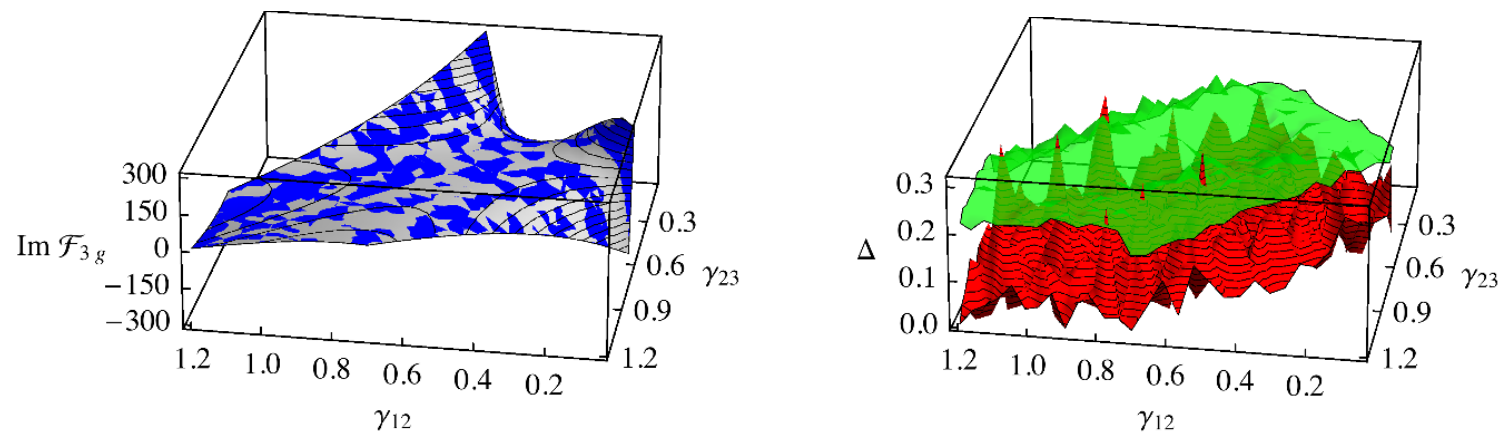

Figure 16. (Color online.) A comparison between the numerical and analytical results for the imaginary part of the three-gluon vertex diagram. The left pane shows $\operatorname{Im} \mathcal{F}_{3 g}$ as a function of two cusp angles from numerical integration (in blue) and from the analytical result (in light gray) superimposed. The numerical result fits the analytic function rather well, with an overall scale factor deviating from one by about $2 \cdot 10^{-5}$. In the right pane we observe that $\Delta$, the absolute difference between the numerical and the analytical values, (in red) is below the numerical errors (in translucent green) for nearly all points $\left(\gamma_{12}, \gamma_{23}\right)$ and is on average about three times smaller. The relative difference with respect to the analytic formula, $\Delta /\left|\operatorname{Im} \mathcal{F}_{3 g}\right|$, is on average of the order of 2 percent, leading to the conclusion that there is excellent agreement between the numerical and analytical results. In both plots $\gamma_{13}=0.5$, while $\gamma_{12}$ and $\gamma_{23}$ vary between 0.0 and 1.2.

the antisymmetry must be recovered after integration (cf. eq. (5.1)). At the level of numerical integration this indeed happens for small cusp angles, while for large cusp angles numerical instabilities arise from the integration near $z \approx 1$, i.e. very large $\psi$. Averaging over the cusp angles $\gamma_{i j}$ remedies those instabilities.

Having constructed expressions for $\zeta_{i}$ in terms of $z$, the angles $\vartheta, \phi$ and the cusp angles $\gamma_{i j}$, the imaginary part of the three-gluon vertex diagram is explicitly given by

$$
\operatorname{Im} \mathcal{F}_{3 g}=\int_{0}^{1} \frac{\mathrm{d} z z^{2}}{\left(1-z^{2}\right)^{2}} \int_{0}^{\pi} \mathrm{d} \vartheta \sin \vartheta \int_{0}^{2 \pi} \mathrm{d} \phi \sum_{i, j, k=1}^{3} \varepsilon_{i j k} v_{i} \cdot v_{j} \zeta_{i} \zeta_{k}\left(\frac{\partial}{\partial \zeta_{i}} \mathcal{G}\left(\left\{\zeta_{i}\right\}\right)\right)
$$

where the cut operators are absorbed into the function $\mathcal{G}\left(\left\{\zeta_{i}\right\}\right)$, given by

$$
\begin{aligned}
\mathcal{G}\left(\left\{\zeta_{i}\right\}\right) & \equiv\left(\operatorname{Cut}_{x_{i}}+\mathrm{Cut}_{x_{j}}+\mathrm{Cut}_{x_{k}}-\mathrm{Cut}_{x_{i}, x_{j}, x_{k}}\right) g\left(\zeta_{i}, 0\right) g\left(\zeta_{j}, 0\right) g\left(\zeta_{k}, 0\right) \\
& =-\frac{\pi \operatorname{arcosh} \zeta_{j} \operatorname{arcosh} \zeta_{k}+\pi \operatorname{arcosh} \zeta_{i} \operatorname{arcosh} \zeta_{k}+\pi \operatorname{arcosh} \zeta_{i} \operatorname{arcosh} \zeta_{j}-\pi^{3}}{\left(\zeta_{i}^{2}-1\right)^{1 / 2}\left(\zeta_{j}^{2}-1\right)^{1 / 2}\left(\zeta_{k}^{2}-1\right)^{1 / 2}} .
\end{aligned}
$$

For the numerical integration of eq. (5.12) we have used GSL [81]. A comparison between the numerical and analytical results for $\operatorname{Im} \mathcal{F}_{3 g}$ is shown in figure 16 . We find that the relative difference between the numerical and analytical results are at the percent level, with absolute differences smaller than numerical errors. We conclude that our formula for the imaginary part in eq. (5.12) is in excellent agreement with the analytic expression in eq. (5.2). This suggests the applicability of the formalism introduced in ref. [1] to obtain the imaginary part of any eikonal diagram with internal vertices. 


\section{Conclusions}

In this paper we have provided algorithms for the compution of the position-space cuts of eikonal diagrams introduced in ref. [1] and discussed the interplay of the cutting prescription with non-Abelian exponentiation. The cutting prescription is applied directly to the position-space representation of an eikonal diagram and computes its imaginary part to the leading order in the dimensional regulator $\epsilon$. The prescription is stated in eqs. (3.10)(3.12). The relation of the imaginary part to the branch cut discontinuity is given in eq. (3.14).

Momentum-space cuts of eikonal diagrams, analogous to the Cutkosky rules for standard Feynman diagrams, were introduced in ref. [21] where they were used to show that the exchanges of Glauber-region gluons (i.e., maximally transverse gluons) produce imaginary parts of the Wilson-line correlator. Any given momentum-space cut separates the eikonal diagram into two disjoint subdiagrams, putting the eikonal and, depending on the cut, possibly also a number of standard Feynman propagators on shell (see figure 5 for an illustration). As a result, momentum-space cuts have the conceptual advantage of factoring eikonal diagrams into on-shell lower-loop and tree diagrams which can be computed as independent objects. In practice, however, the resulting cut diagrams involve integrations over two-, three-, four-, ... particle phase space. The evaluation of these phase-space integrals poses a substantial computational challenge, limiting the applicability of momentum-space cuts for computing imaginary parts.

In contrast, position-space cuts do not factor the eikonal diagram into disjoint subdiagrams, but rather constrain the gauge bosons exchanged between the energetic partons to be lightlike. For space-like external kinematics such exchanges are causally impossible, and the imaginary part vanishes. For time-like kinematics such exchanges are allowed and generate a nontrivial evolution of the phases of the parton states, leading in turn to a close relation of the imaginary part of the cusp anomalous dimension to the static interquark potential. Position space thus offers a causality viewpoint on the origin of the imaginary part of the eikonal diagram. This is complementary to the unitarity viewpoint provided by momentum space - i.e., that the imaginary part arises from the hard partons going on shell and exchanging Glauber-region gluons. At the computational level, the number of position-space cut diagrams contributing to the imaginary part of a given eikonal diagram is in practice smaller than the number of momentum-space cut contributions, and several of the position-space cut diagrams can be seen to be equal a priori.

We have applied our formalism to several two- and three-loop eikonal diagrams, finding agreement with results previously obtained in the literature [21, 28, 35, 38, 39]. These computations also serve to demonstrate that the position-space cut diagrams contributing to the imaginary part of a given eikonal diagram can be evaluated in practice in nontrivial cases. In particular, for eikonal diagrams without internal vertices - i.e., QED-like diagrams - the contributing cut diagrams can be evaluated systematically by means of our algorithm for computing the principal-value integrals involved (supplemented with a slight generalization of the algorithm of that in ref. [76] for expressing multiple polylogarithms in terms of ones with constant indices). 
The formalism developed in this paper allows us to compute the imaginary part of eikonal diagrams only to the leading order in $\epsilon$. This appears to limit the applicability of the approach, but in practice Wilson line correlators can often be expressed in terms of diagrams with simple poles in $\epsilon$ (once the diagrams are expressed in terms of the renormalized coupling). This owes to the non-Abelian exponentiation theorem [4, 5, 8-14] which states that the correlator can be expressed as the exponential of specific linear combinations of diagrams mutually related by permutations of the soft-gluon attachment points. These linear combinations, called webs, have the property that the leading divergence of the constituent diagrams cancels, leaving in many cases webs with simple poles in $\epsilon$. The organization of the exponent of the Wilson line correlator in terms of webs is particularly beneficial for the applicability of the present cutting prescription: the cuts must be applied to an entire web rather than its constituent diagrams separately, as the separate imaginary parts, computed to leading order in $\epsilon$, will cancel. In this sense the cutting prescription has a nontrivial interplay with non-Abelian exponentiation.

It would be intriguing to investigate whether the position-space cuts studied in this paper can be utilized, or serve as inspiration, for developing (generalized) unitarity methods $[48-52,54-57]$ for correlators of Wilson lines. Another interesting direction for future research is the extension of the present formalism to computations of imaginary parts of Wilson line correlators to subleading orders in $\epsilon$.

\section{Acknowledgments}

We thank Samuel Abreu, Simon Caron-Huot, Einan Gardi, Johannes Henn, Paul Hoyer, Lorenzo Magnea, George Sterman, Iain Stewart, Ward Vleeshouwers, Andries Waelkens, Chris White and especially Gregory Korchemsky for useful discussions. We are grateful for the hospitality of the Higgs Centre of the University of Edinburgh. KJL is grateful for the hospitality of the Institute for Advanced Study in Princeton and the Institut de Physique Théorique, CEA Saclay, where part of this work was carried out. The research leading to these results has received funding from the European Union Seventh Framework Programme (FP7/2007-2013) under grant agreement no. 627521. This work was supported by the Foundation for Fundamental Research of Matter (FOM), program 104 "Theoretical Particle Physics in the Era of the LHC" and by the Research Executive Agency (REA) of the European Union under the Grant Agreement number PITN-GA-2010-264564 (LHCPhenoNet) and PITN-GA-2012-316704 (HIGGSTOOLS).

\section{A Real and imaginary parts of multiple polylogarithms}

The position-space cut prescription in eq. (3.12) produces principal-value integrals when applied to eikonal diagrams beyond one loop. In practice, we compute such integrals as the corresponding full integral (which evaluates into multiple polylogarithms) minus its imaginary part, cf. eq. (2.10). In this appendix we describe how to construct the required real and imaginary part of multiple polylogarithms to arbitrary weight in a systematic way. 
Explicit formulas for imaginary parts are given up to weight four, while the real parts are obtained by subtracting the imaginary part from the original function.

Let us first introduce some notation. Multiple polylogarithms are defined recursively by

$$
G\left(a_{1}, \ldots, a_{n} ; x\right)=\int_{0}^{x} \frac{\mathrm{d} t}{t-a_{1}} G\left(a_{2}, \ldots, a_{n} ; t\right) \quad \text { for }\left(a_{1}, \ldots, a_{n}\right) \neq \overrightarrow{0}_{n},
$$

starting from the special cases

$$
G(; 0) \equiv 0, \quad G(; x) \equiv 1, \quad G\left(\overrightarrow{0}_{n} ; x\right) \equiv \frac{1}{n !} \log ^{n} x
$$

where $\vec{a}_{n}=(a, \ldots, a)$ denotes a vector with $n$ equal indices. Multiple polylogarithms satisfy a variety of properties. They form a shuffle algebra,

$$
G\left(a_{1}, \ldots, a_{n_{1}} ; x\right) G\left(a_{n_{1}+1}, \ldots, a_{n_{1}+n_{2}} ; x\right)=\sum_{\sigma \in \Sigma\left(n_{1}, n_{2}\right)} G\left(a_{\sigma(1)}, \ldots, a_{\sigma\left(n_{1}+n_{2}\right)} ; x\right) .
$$

They are invariant under a common rescaling of all arguments: setting $\vec{a}=\left(a_{1}, \ldots, a_{n}\right)$ we have

$$
G(k \vec{a} ; k x)=G(\vec{a} ; x) \quad \text { for } a_{i} \neq 0 \text { and } k \in \mathbb{C}^{*} .
$$

They reduce to classical polylogarithms in certain cases,

$$
\begin{aligned}
G\left(\overrightarrow{0}_{n} ; x\right) & =\frac{1}{n !} \log ^{n} x, & G\left(\vec{a}_{n} ; x\right) & =\frac{1}{n !} \log ^{n}\left(1-\frac{x}{a}\right), \\
G\left(\overrightarrow{0}_{n-1}, a ; x\right) & =-\operatorname{Li}_{n}\left(\frac{x}{a}\right), & G\left(\overrightarrow{0}_{n}, \vec{a}_{p} ; x\right) & =(-1)^{p} S_{n, p}\left(\frac{x}{a}\right),
\end{aligned}
$$

or to harmonic polylogarithms, introduced in ref. [82],

$$
G(\vec{a} ; x)=(-1)^{k} H_{\vec{a}}(x) \quad \text { if } \quad \forall a_{i} \in \vec{a}: a_{i} \in\{ \pm 1,0\}
$$

where $k$ denotes the number of +1 's in $\vec{a}$. We refer to ref. [74] for more details.

Having set the notation, we turn to the problem of constructing the real and imaginary parts of multiple polylogarithms. As it turns out, it is most convenient to obtain the real part as the difference of the full function and its imaginary part,

$$
\operatorname{Re} G(\vec{a} ; x)=G(\vec{a} ; x)-i \operatorname{Im} G(\vec{a} ; x) .
$$

Thus, it suffices to determine the imaginary part. In the remainder of this section we accordingly focus on the construction of imaginary parts.

The imaginary part of a multiple polylogarithm arises when one or more of its indices $a_{i}$ are located along the path of integration of the corresponding iterated integral, that is $a_{i} \in[0, x]$. By giving either the argument or the indices an infinitesimal imaginary part, the imaginary part of any multiple polylogarithm is fixed recursively in terms of the imaginary part of the classical logarithm. This is most easily seen in the special case of a polylogarithm with all indices equal to zero by using its definition in eq. (A.2) in terms of logarithms,

$$
\operatorname{Im} G\left(\overrightarrow{0}_{n} ; x \pm i \eta\right)=\frac{1}{n !} \operatorname{Im} \log ^{n}(x \pm i \eta)=\frac{1}{n !} \operatorname{Im}\left[(\log |x| \pm i \pi \theta(-x))^{n}\right] .
$$


The imaginary part on the right-hand side may be obtained by simply expanding the product and collecting the terms proportional to $i$. The other two special cases in eq. (A.2) are real constants and thus have a vanishing imaginary part. This concludes the computation of the imaginary part of all the special cases listed in eq. (A.2).

We thus turn to determining the imaginary part of a multiple polylogarithm in the general case $G(\vec{a} ; x)$ with at least one non-zero index, cf. eq. (A.1). Since an imaginary part arises when some of the indices $a_{i}$ are located along the path of integration, it will be necessary to know the relative locations of the $a_{i}$ 's in the complex plane. To this end, we will make a few assumptions on the indices and arguments of $G(\vec{a} ; x)$. First, let us observe that all polylogarithms encountered in this paper will have real indices $a_{i}$ (up to an infinitesimal imaginary part whose sign is fixed by the Feynman rules). In addition, we will assume, without loss of generality, that the last index is non-zero, and that the endpoint of integration $x$ is real and positive.

The fact that the latter two assumptions may be imposed without loss of generality follows from the properties of multiple polylogarithms. Indeed, multiple polylogarithms with any number of trailing zeros may be expressed, with the help of the shuffle algebra in eq. (A.3), in terms of multiple polylogarithms with a non-zero last index, multiplied by pure logarithms. For example,

$$
\begin{aligned}
G(a, 0 ; x) & =G(a ; x) G(0 ; x)-G(0, a ; x), \\
G(a, 0,0 ; x) & =G(a ; x) G(0,0 ; x)-G(0, a, 0 ; x)-G(0,0, a ; x) \\
& =G(a ; x) G(0,0 ; x)-G(0, a ; x) G(0 ; x)+G(0,0, a ; x) .
\end{aligned}
$$

Their imaginary part is thus given in terms of the (real and) imaginary parts of multiple polylogarithms with a non-zero last index and pure logarithms. The latter are known from eq. (A.8). Thus, it suffices to determine the imaginary part of polylogarithms with a non-zero last index.

Now, taking the last index to be non-zero, we may apply the rescaling relation eq. (A.4) with $k=-1$,

$$
G(\vec{a} ; x)=G(-\vec{a} ;-x) \text { for } a_{n} \neq 0
$$

to map any negative argument $x$ to a positive argument [83]. Likewise, a complex argument can be mapped to a real number after rescaling by $k=1 / x$, yielding $G(\vec{a} / x ; 1)$.

Our task is thus to determine the imaginary part of a multiple polylogarithm in the general case $G(\vec{a} ; x)$ with a non-zero last index, and with the endpoint of integration $x$ being real and positive. Starting at weight one, we let $a, x \in \mathbb{R}, a \neq 0$ and $x>0$. Explicit computation of the imaginary part - using the fact that the imaginary part of an integral equals the integral over the imaginary part — yields

$$
\operatorname{Im} G(a \pm i \eta ; x)=\operatorname{Im} \int_{0}^{x} \frac{\mathrm{d} t}{t-(a \pm i \eta)}= \pm \pi \int_{0}^{x} \mathrm{~d} t \delta(t-a)= \pm \pi \theta(a) \theta(x-a) .
$$

This is the explicit formula for the imaginary part at lowest weight. 
Moving on to higher-weight polylogarithms, let us consider a weight $n$ multiple polylogarithm. The integrand takes the form

$$
\mathcal{I}=\frac{1}{t_{1}-\left(a_{1} \pm i \eta\right)} \frac{1}{t_{2}-\left(a_{2} \pm i \eta\right)} \cdots \frac{1}{t_{n}-\left(a_{n} \pm i \eta\right)}
$$

The imaginary part of $\mathcal{I}$ may be broken into real and imaginary parts of each of the above fractions. The imaginary part of a single fraction is simple, because it localizes the corresponding integration variable to a point. In contrast, the real parts do not simplify, so it is convenient to have as few real-part evaluations as possible. To this end, we split the imaginary part of the integrand (A.12) into products of imaginary parts and real parts of products by recursively applying

$$
\begin{aligned}
\operatorname{Im}(P Q) & =\operatorname{Im} P \operatorname{Re} Q+\operatorname{Re} P \operatorname{Im} Q, \\
\operatorname{Re} P \operatorname{Re} Q & =\operatorname{Re}(P Q)+\operatorname{Im} P \operatorname{Im} Q,
\end{aligned}
$$

where $P$ and $Q$ represent either a single fraction or products of fractions. For example,

$$
\begin{aligned}
\operatorname{Im}(a b)= & \operatorname{Im} a \operatorname{Re} b+\operatorname{Im} b \operatorname{Re} a \\
\operatorname{Im}(a b c)= & \operatorname{Im} a \operatorname{Re}(b c)+\operatorname{Im} b \operatorname{Re}(a c)+\operatorname{Im} c \operatorname{Re}(a b)+2 \operatorname{Im} a \operatorname{Im} b \operatorname{Im} c, \\
\operatorname{Im}(a b c d)= & \operatorname{Im} a \operatorname{Re}(b c d)+\operatorname{Im} b \operatorname{Re}(a c d)+\operatorname{Im} c \operatorname{Re}(a b d)+\operatorname{Im} d \operatorname{Re}(a b c)+2 \operatorname{Im} a \operatorname{Im} b \operatorname{Im} c \operatorname{Re} d \\
& +2 \operatorname{Im} a \operatorname{Im} b \operatorname{Im} d \operatorname{Re} c+2 \operatorname{Im} a \operatorname{Im} c \operatorname{Im} d \operatorname{Re} b+2 \operatorname{Im} b \operatorname{Im} c \operatorname{Im} d \operatorname{Re} a,
\end{aligned}
$$

where $a, b, c$ and $d$ represent individual fractions in the integrand $\mathcal{I}$ of a multiple polylogarithm. Notice that each term on the right-hand side of eq. (A.14) contains only one real part (of a product) and a product of imaginary parts (of single factors).

The imaginary part of higher-weight multiple polylogarithms are then computed by applying eq. (A.14) to the integrand and integrating out the delta functions arising from eq. (2.10). For example, the imaginary part of a weight-two multiple polylogarithm for $a, b, x \in \mathbb{R}, b \neq 0$ and $x>0$ is computed as follows,

$$
\begin{aligned}
\operatorname{Im} & G(a \pm i \eta, b \pm i \eta ; x) \\
= & \int_{0}^{x} \mathrm{~d} t \int_{0}^{t} \mathrm{~d} u \operatorname{Im}\left(\frac{1}{t-(a \pm i \eta)} \frac{1}{u-(b \pm i \eta)}\right) \\
= & \int_{0}^{x} \mathrm{~d} t \int_{0}^{t} \mathrm{~d} u\left[\operatorname{Im}\left(\frac{1}{t-(a \pm i \eta)}\right) \operatorname{Re}\left(\frac{1}{u-(b \pm i \eta)}\right)+\operatorname{Re}\left(\frac{1}{t-(a \pm i \eta)}\right) \operatorname{Im}\left(\frac{1}{u-(b \pm i \eta)}\right)\right] \\
= & \pm \pi \int_{0}^{x} \mathrm{~d} t \delta(t-a) \int_{0}^{t} \mathrm{~d} u \operatorname{Re}\left(\frac{1}{u-(b \pm i \eta)}\right) \pm \pi \int_{0}^{x} \mathrm{~d} t \operatorname{Re}\left(\frac{1}{t-(a \pm i \eta)}\right) \int_{0}^{t} \mathrm{~d} u \delta(u-b) \\
= & \pm \pi \theta(a) \theta(x-a) \operatorname{Re} \int_{0}^{a} \frac{\mathrm{d} u}{u-(b \pm i \eta)} \pm \pi \operatorname{Re} \int_{0}^{x} \frac{\mathrm{d} t}{t-(a \pm i \eta)} \theta(b) \theta(t-b) \\
& = \pm \pi \theta(a) \theta(x-a) \operatorname{Re} G(b \pm i \eta ; a) \pm \pi \theta(b) \theta(x-b) \operatorname{Re}(G(a \pm i \eta ; x)-G(a \pm i \eta ; b)) .
\end{aligned}
$$

The real parts of lower-weight functions on the right-hand side are known inductively from expressions for lower-weight imaginary parts, together with eq. (A.7) for the real part. We 
remark that when $a=0$, the first term in eq. (A.15) vanishes because $G(b \pm i \eta ; 0)=0$. The imaginary part of weight-three and -four multiple polylogarithms have been computed along the same lines. Before quoting the results, it is advantageous to introduce some notation, in terms of which the formulas assume a nice form.

The imaginary parts are conveniently expressed in terms of two new functions: a slightly different notation for multiple polylogarithms, together with generalized step functions. Let us first introduce the former, which is an iterated integral where the base point of integration may be freely chosen,

$$
I\left(a_{0} ; a_{1}, \ldots, a_{n} ; a_{n+1}\right)=\int_{a_{0}}^{a_{n+1}} \frac{\mathrm{d} t}{t-a_{n}} I\left(a_{0} ; a_{1}, \ldots, a_{n-1} ; t\right) \quad \text { with } I\left(a_{0} ; ; x\right) \equiv 1
$$

Setting the base point to zero we obviously recover the multiple polylogarithms defined in eq. (A.1), up to a conventional reversal of the indices. Although eq. (A.16) appears to define a larger class of integrals, it actually does not. Any $I$ can be written as a linear combination of (products of) G's. This is achieved by splitting the range of integration into a difference of paths with basepoint zero, cf. ref. [75]. For example,

$$
I\left(a_{0} ; a_{1} ; a_{2}\right)=I\left(0 ; a_{1} ; a_{2}\right)-I\left(0 ; a_{1} ; a_{0}\right)=G\left(a_{1} ; a_{2}\right)-G\left(a_{1} ; a_{0}\right) .
$$

At higher weight one splits the innermost integrals first. At weight two,

$$
\begin{aligned}
I\left(a_{0} ; a_{1}, a_{2} ; a_{3}\right) & =\int_{a_{0}}^{a_{3}} \frac{\mathrm{d} t}{t-a_{2}}\left(G\left(a_{1} ; t\right)-G\left(a_{1} ; a_{0}\right)\right) \\
& =G\left(a_{2}, a_{1} ; a_{3}\right)-G\left(a_{2}, a_{1} ; a_{0}\right)-G\left(a_{1} ; a_{0}\right)\left(G\left(a_{2} ; a_{3}\right)-G\left(a_{2} ; a_{0}\right)\right) .
\end{aligned}
$$

In this way any $I$ can be written in terms of $G$ 's.

We also introduce generalized step functions $\theta\left(a_{1}, \ldots, a_{n}\right)$, which may be thought of as enforcing $a_{1} \leq \cdots \leq a_{n}$. In terms of ordinary single-variable step functions,

$$
\theta\left(a_{1}, \ldots, a_{n}\right) \equiv \prod_{i=1}^{n-1} \theta\left(a_{i+1}-a_{i}\right) \quad \text { for } n>1 \text { and } \forall i: a_{i} \neq a_{i+1} \text { and } a_{i} \in \mathbb{R}
$$

Equal adjacent arguments are dealt with using the following definition

$$
\theta(\ldots, a, \underbrace{b, \ldots, b}_{n \text { times }}, c, \ldots)=\frac{1}{n !} \theta(\ldots, a, b, c, \ldots) \quad \text { for real indices } a \neq b \neq c .
$$

Infinitesimal imaginary parts produce an overall sign,

$$
\theta(\ldots, a \pm i \eta, \ldots)= \pm \theta(\ldots, a, \ldots) \quad \text { for } a \in \mathbb{R}
$$


In this notation, the imaginary part of multiple polylogarithms up to weight four are

$$
\begin{aligned}
\operatorname{Im} G(a ; x)= & \pi \theta(0, a, x) \\
\operatorname{Im} G(a, b ; x)= & \pi \theta(0, a, x) \operatorname{Re} G(b ; a)+\pi \theta(0, b, x) \operatorname{Re} I(b ; a ; x) \\
\operatorname{Im} G(a, b, c ; x)= & \pi \theta(0, a, x) \operatorname{Re} G(b, c ; a)+\pi \theta(0, b, x) \operatorname{Re}[I(b ; a ; x) G(c ; b)] \\
& +\pi \theta(0, c, x) \operatorname{Re} I(c ; b, a ; x)+2 \pi^{3} \theta(0, c, b, a, x) \\
\operatorname{Im} G(a, b, c, d ; x)= & \pi \theta(0, a, x) \operatorname{Re} G(b, c, d ; a) \\
& +\pi \theta(0, b, x) \operatorname{Re}[I(b ; a ; x) G(c, d ; b)] \\
& +\pi \theta(0, c, x) \operatorname{Re}[I(c ; b, a ; x) G(d ; c)] \\
& +\pi \theta(0, d, x) \operatorname{Re} I(d ; c, b, a ; x) \\
& +2 \pi^{3} \theta(0, c, b, a, x) \operatorname{Re} G(d ; c) \\
& +2 \pi^{3} \theta(0, d, b, a, x) \operatorname{Re} I(d ; c ; b) \\
& +2 \pi^{3} \theta(0, d, c, a, x) \operatorname{Re} I(c ; b ; a) \\
& +2 \pi^{3} \theta(0, d, c, b, x) \operatorname{Re} I(b ; a ; x)
\end{aligned}
$$

where $a, b, c, d, x \in \mathbb{R}, x$ is positive, and in each case the last index is non-zero. The suppressed Feynman $i \eta$ 's may be reinstated by replacing the indices according to $a \rightarrow a \pm i \eta$.

We conclude this section by providing proofs of eqs. (A.23) and (A.24).

Proof of eq. (A.23). We proceed by direct computation, using eq. (A.14) and the identity $\operatorname{Im} \frac{1}{\xi \pm i 0}=\mp \pi \delta(\xi)$

$$
\begin{aligned}
\operatorname{Im} G(a, b ; x) & =\operatorname{Im}\left(\int_{0}^{x} \frac{\mathrm{d} t}{t-a-i 0} \int_{0}^{t} \frac{\mathrm{d} u}{u-b-i 0}\right) \\
& =\int_{0}^{x} \mathrm{~d} t \int_{0}^{t} \mathrm{~d} u \operatorname{Im}\left(\frac{1}{t-a-i 0} \frac{1}{u-b-i 0}\right) \\
& =\int_{0}^{x} \mathrm{~d} t \int_{0}^{t} \mathrm{~d} u\left[\operatorname{Im}\left(\frac{1}{t-a-i 0}\right) \operatorname{Re}\left(\frac{1}{u-b-i 0}\right)+\operatorname{Re}\left(\frac{1}{t-a-i 0}\right) \operatorname{Im}\left(\frac{1}{u-b-i 0}\right)\right] \\
& =\int_{0}^{x} \mathrm{~d} t \int_{0}^{t} \mathrm{~d} u\left[\pi \delta(t-a) \operatorname{Re}\left(\frac{1}{u-b-i 0}\right)+\operatorname{Re}\left(\frac{1}{t-a-i 0}\right) \pi \delta(u-b)\right] \\
& =\pi \int_{0}^{x} \mathrm{~d} t \delta(t-a) \int_{0}^{t} \mathrm{~d} u \operatorname{Re}\left(\frac{1}{u-b-i 0}\right)+\pi \int_{0}^{x} \mathrm{~d} t \operatorname{Re}\left(\frac{1}{t-a-i 0}\right) \int_{0}^{t} \mathrm{~d} u \delta(u-b) \\
& =\pi \theta(0, a, x) \int_{0}^{a} \mathrm{~d} u \operatorname{Re}\left(\frac{1}{u-b-i 0}\right)+\pi \int_{0}^{x} \mathrm{~d} t \operatorname{Re}\left(\frac{1}{t-a-i 0}\right) \theta(0, b, t) \\
& =\pi \theta(0, a, x) \operatorname{Re}\left(\int_{0}^{a} \frac{\mathrm{d} u}{u-b-i 0}\right)+\pi \theta(0, b, x) \operatorname{Re}\left(\int_{b}^{x} \frac{\mathrm{d} t}{t-a-i 0}\right) \\
& =\pi \theta(0, a, x) \operatorname{Re} G(b ; a)+\pi \theta(0, b, x) \operatorname{Re} I(b ; a ; x) .
\end{aligned}
$$

This completes the proof of eq. (A.23). 
Proof of eq. (A.24). We proceed by direct computation, using eq. (A.14) and the identity $\operatorname{Im} \frac{1}{\xi \pm i 0}=\mp \pi \delta(\xi)$,

$$
\begin{aligned}
\operatorname{Im} & G(a, b, c ; x) \\
= & \operatorname{Im}\left(\int_{0}^{x} \frac{\mathrm{d} t}{t-a-i 0} \int_{0}^{t} \frac{\mathrm{d} u}{u-b-i 0} \int_{0}^{u} \frac{\mathrm{d} v}{v-c-i 0}\right) \\
= & \int_{0}^{x} \mathrm{~d} t \int_{0}^{t} \mathrm{~d} u \int_{0}^{u} \mathrm{~d} v \operatorname{Im}\left(\frac{1}{t-a-i 0} \frac{1}{u-b-i 0} \frac{1}{v-c-i 0}\right) \\
= & \int_{0}^{x} \mathrm{~d} t \int_{0}^{t} \mathrm{~d} u \int_{0}^{u} \mathrm{~d} v\left[\operatorname{Im}\left(\frac{1}{t-a-i 0}\right) \operatorname{Re}\left(\frac{1}{u-b-i 0} \frac{1}{v-c-i 0}\right)\right. \\
& \quad+\operatorname{Im}\left(\frac{1}{u-b-i 0}\right) \operatorname{Re}\left(\frac{1}{t-a-i 0} \frac{1}{v-c-i 0}\right)+\operatorname{Im}\left(\frac{1}{v-c-i 0}\right) \operatorname{Re}\left(\frac{1}{t-a-i 0} \frac{1}{u-b-i 0}\right) \\
& \left.\quad+2 \operatorname{Im}\left(\frac{1}{t-a-i 0}\right) \operatorname{Im}\left(\frac{1}{u-b-i 0}\right) \operatorname{Im}\left(\frac{1}{v-c-i 0}\right)\right] \\
= & \int_{0}^{x} \mathrm{~d} t \int_{0}^{t} \mathrm{~d} u \int_{0}^{u} \mathrm{~d} v\left[\pi \delta(t-a) \operatorname{Re}\left(\frac{1}{u-b-i 0} \frac{1}{v-c-i 0}\right)+\pi \delta(u-b) \operatorname{Re}\left(\frac{1}{t-a-i 0} \frac{1}{v-c-i 0}\right)\right. \\
& \left.\quad+\pi \delta(v-c) \operatorname{Re}\left(\frac{1}{t-a-i 0} \frac{1}{u-b-i 0}\right)+2 \pi^{3} \delta(t-a) \delta(u-b) \delta(v-c)\right] \\
\equiv & I_{(3,1)}+I_{(3,2)}+I_{(3,3)}+I_{(3,4)} .
\end{aligned}
$$

We continue by evaluating each term in the last line of eq. (A.27) separately. The first term evaluates to,

$$
\begin{aligned}
I_{(3,1)} & =\pi \int_{0}^{x} \mathrm{~d} t \delta(t-a) \int_{0}^{t} \mathrm{~d} u \int_{0}^{u} \mathrm{~d} v \operatorname{Re}\left(\frac{1}{u-b-i 0} \frac{1}{v-c-i 0}\right) \\
& =\pi \theta(0, a, x) \int_{0}^{a} \mathrm{~d} u \int_{0}^{u} \mathrm{~d} v \operatorname{Re}\left(\frac{1}{u-b-i 0} \frac{1}{v-c-i 0}\right) \\
& =\pi \theta(0, a, x) \operatorname{Re}\left(\int_{0}^{a} \mathrm{~d} u \int_{0}^{u} \mathrm{~d} v \frac{1}{u-b-i 0} \frac{1}{v-c-i 0}\right) \\
& =\pi \theta(0, a, x) \operatorname{Re} G(b, c ; a) .
\end{aligned}
$$

The second term in eq. (A.27) evaluates to,

$$
\begin{aligned}
I_{(3,2)} & =\pi \int_{0}^{x} \mathrm{~d} t \int_{0}^{t} \mathrm{~d} u \delta(u-b) \int_{0}^{u} \mathrm{~d} v \operatorname{Re}\left(\frac{1}{t-a-i 0} \frac{1}{v-c-i 0}\right) \\
& =\pi \int_{0}^{x} \mathrm{~d} t \theta(0, b, t) \int_{0}^{b} \mathrm{~d} v \operatorname{Re}\left(\frac{1}{t-a-i 0} \frac{1}{v-c-i 0}\right) \\
& =\pi \theta(0, b, x) \int_{b}^{x} \mathrm{~d} t \int_{0}^{b} \mathrm{~d} v \operatorname{Re}\left(\frac{1}{t-a-i 0} \frac{1}{v-c-i 0}\right) \\
& =\pi \theta(0, b, x) \operatorname{Re}\left(\int_{b}^{x} \mathrm{~d} t \frac{1}{t-a-i 0} \int_{0}^{b} \mathrm{~d} v \frac{1}{v-c-i 0}\right) \\
& =\pi \theta(0, b, x) \operatorname{Re}(I(b ; a ; x) G(c ; b)) .
\end{aligned}
$$


The third term in eq. (A.27) evaluates to,

$$
\begin{aligned}
I_{(3,3)} & =\pi \int_{0}^{x} \mathrm{~d} t \int_{0}^{t} \mathrm{~d} u \int_{0}^{u} \mathrm{~d} v \delta(v-c) \operatorname{Re}\left(\frac{1}{t-a-i 0} \frac{1}{u-b-i 0}\right) \\
& =\pi \int_{0}^{x} \mathrm{~d} t \int_{0}^{t} \mathrm{~d} u \theta(0, c, u) \operatorname{Re}\left(\frac{1}{t-a-i 0} \frac{1}{u-b-i 0}\right) \\
& =\pi \int_{0}^{x} \mathrm{~d} t \theta(0, c, t) \int_{c}^{t} \mathrm{~d} u \operatorname{Re}\left(\frac{1}{t-a-i 0} \frac{1}{u-b-i 0}\right) \\
& =\pi \theta(0, c, x) \int_{c}^{x} \mathrm{~d} t \int_{c}^{t} \mathrm{~d} u \operatorname{Re}\left(\frac{1}{t-a-i 0} \frac{1}{u-b-i 0}\right) \\
& =\pi \theta(0, c, x) \operatorname{Re}\left(\int_{c}^{x} \mathrm{~d} t \int_{c}^{t} \mathrm{~d} u \frac{1}{t-a-i 0} \frac{1}{u-b-i 0}\right) \\
& =\pi \theta(0, c, x) \operatorname{Re} I(c ; b, a ; x) .
\end{aligned}
$$

The fourth term in eq. (A.27) evaluates to,

$$
\begin{aligned}
I_{(3,4)} & =2 \pi^{3} \int_{0}^{x} \mathrm{~d} t \int_{0}^{t} \mathrm{~d} u \int_{0}^{u} \mathrm{~d} v \delta(t-a) \delta(u-b) \delta(v-c) \\
& =2 \pi^{3} \int_{0}^{x} \mathrm{~d} t \delta(t-a) \int_{0}^{t} \mathrm{~d} u \delta(u-b) \int_{0}^{u} \mathrm{~d} v \delta(v-c) \\
& =2 \pi^{3} \int_{0}^{x} \mathrm{~d} t \delta(t-a) \int_{0}^{t} \mathrm{~d} u \delta(u-b) \theta(0, c, u) \\
& =2 \pi^{3} \int_{0}^{x} \mathrm{~d} t \delta(t-a) \theta(0, c, b, t) \\
& =2 \pi^{3} \theta(0, c, b, a, x) .
\end{aligned}
$$

Adding up the four contributions in eqs. (A.28)-(A.31) according to eq. (A.27) we obtain the result given in eq. (A.24). This completes the proof.

The identity (A.25) may be shown by completely analogous steps, and we therefore omit its proof here.

\section{B Algorithm for achieving canonical-form polylogarithms}

In their position-space representation, eikonal diagrams without internal vertices, and the corresponding cut diagrams, take the form of iterated integrals. A first step in the evaluation of these diagrams therefore amounts to recognizing the definition of multiple polylogarithms in terms of iterated integrals, cf. eq. (A.1). The resulting multiple polylogarithms depend in general on the kinematical variables of the problem through both their indices and their argument. They may be rewritten in terms of polylogarithms with constant indices, in so-called canonical form, by exploiting the Hopf algebra structure of multiple polylogarithms, which encodes the plethora of functional relations within this class of functions. In this appendix we describe the algorithm to cast multiple polylogarithms in canonical form, which is extensively used in the computations in section 4 . 
The algorithm to cast multiple polylogarithms in canonical form relies on the Hopf algebra structure of multiple polylogarithms. In particular, we make use of the notions of the symbol and coproduct of multiple polylogarithms, see refs. [74, 75] and references therein, as well as a procedure from ref. [76] to map symbols to polylogarithms. In order to describe the algorithm, we start by setting up notation. Let us denote an eikonal diagram, or a cut eikonal diagram (or a partially integrated result thereof), by the function $g(\vec{x})$, depending on $n$ variables, $\vec{x}=\left\{x_{1}, \ldots, x_{n}\right\}$. (This set of variables typically contains the cusp angles and possibly some remaining integration variables.) After recognizing the definition of multiple polylogarithms, the function $g(\vec{x})$ is given in terms of functions $G(\vec{a}(\vec{x}) ; z(\vec{x}))$ depending on $\vec{x}$ through both their indices $\vec{a}(\vec{x})$ and their argument $z(\vec{x})$. To simplify the following presentation, we will assume ${ }^{12}$ that $g(\vec{x})$ has uniform transcendental weight $w$.

The algorithm follows three steps. Let us first state the algorithm and subsequently elaborate on each of the steps separately.

\section{Algorithm (Multiple polylogarithms in canonical form)}

1. Compute the symbol $S[g(\vec{x})]$ of the function $g(\vec{x})$.

2. Apply a map $M_{\vec{x}}$ to the symbol $S[g(\vec{x})]$, whose purpose is to construct a polylogarithm in canonical form with the same symbol as the original function. The resulting expression differs only from the original function by terms proportional to transcendental constants (which are in the kernel of the symbol map).

3. Compute subsequently the coproducts $\Delta_{2,1, \ldots, 1}, \Delta_{3,1, \ldots, 1}, \ldots, \Delta_{w-1,1}$ to reconstruct any missing terms proportional to constants with transcendental weight $2,3, \ldots, w-1$ respectively.

The output of this algorithm is a new function $h(\vec{x})$ in canonical form, which is numerically equal to the original function $g(\vec{x})$. In the remainder of this appendix we shall give the definitions of the symbol $S$, the map $M_{\vec{x}}$ and the coproduct $\Delta$. We conclude by illustrating the application of this algorithm to the non-planar two-loop ladder diagram considered in section 4.1.

The first step of the algorithm involves computing the symbol of multiple polylogarithms, first introduced in ref. [84]. The idea of the symbol map is to encode the functional relations among multiple polylogarithms as simple algebraic identities in the corresponding tensor algebra.

One way to define the symbol is by considering the total differential,

$$
\mathrm{d} G\left(a_{n-1}, \ldots, a_{1} ; a_{n}\right)=\sum_{i=1}^{n-1} G\left(a_{n-1}, \ldots, a_{i+1}, a_{i-1}, \ldots, a_{1} ; a_{n}\right) \mathrm{d} \log \left(\frac{a_{i}-a_{i+1}}{a_{i}-a_{i-1}}\right),
$$

\footnotetext{
${ }^{12}$ If this assumption fails, the algorithm may be applied to each subexpressions of uniform weight.
} 
and to define the symbol of a multiple polylogarithm analogously, cf. ref. [72],

$$
S\left[G\left(a_{n-1}, \ldots, a_{1} ; a_{n}\right)\right]=\sum_{i=1}^{n-1} S\left[G\left(a_{n-1}, \ldots, a_{i+1}, a_{i-1}, \ldots, a_{1} ; a_{n}\right)\right] \otimes\left(\frac{a_{i}-a_{i+1}}{a_{i}-a_{i-1}}\right),
$$

in the case of generic indices $a_{i}$; i.e., non-zero and mutually different. The formula for the symbol in eq. (B.2), augmented with formulas for special cases and the rules of symbol calculus (for which we refer the reader to refs. [74, 75]), allows the symbol $S[g(\vec{x})]$ to be computed. This completes the first step of the algorithm.

The second step of the algorithm takes the resulting symbol as input and maps it to an expression of multiple polylogarithms in canonical form, whose symbol is the same as the symbol of the original function. A procedure that achieves this goal was given in appendix D of ref. [76]. We cast their procedure in the form of an explicit map and furthermore make a slight generalization in order to deal with functions of more than two variables which have a sufficiently factorized form. Let us first define the map and then point out wherein the slight generalization resides.

The map $M_{\vec{x}}$ is defined recursively in the number of variables. Starting with the case of a single variable, we define the map $M_{x}$ which takes tensors to functions,

$$
M_{x}(T)= \begin{cases}G\left(-\frac{b_{1}}{a_{1}}, \ldots,-\frac{b_{w}}{a_{w}} ; x\right) & \text { if } T=\left(a_{w} x+b_{w}\right) \otimes \cdots \otimes\left(a_{1} x+b_{1}\right), \\ 0 & \text { otherwise }\end{cases}
$$

where $a_{i}$ and $b_{i}$ are independent of $x$. The map $M_{x}$ is linear in the space of tensors: given a symbol $S=\sum_{i} c_{i} T_{i}$, with rational numbers $c_{i}$ and tensors $T_{i}$, one has $M_{x}(S)=$ $\sum_{i} c_{i} M_{x}\left(T_{i}\right)$. The map $M_{x}(T)$ is designed to construct a function in canonical form, such that its symbol is given by $T$ plus possibly other tensors which have at least one entry independent of $x$. The proof of this statement was given in ref. [76].

Generalizing to the multivariate case, we let $\vec{x}=\left\{x_{1}, \ldots, x_{n}\right\}$ denote a collection of at least two variables, and define the multivariate map

$$
\begin{aligned}
M_{\vec{x}}(S) & =\left(P_{\vec{x}, S}^{(w)} \circ P_{\vec{x}, S}^{(w-1)} \circ \cdots \circ P_{\vec{x}, S}^{(1)} \circ M_{\vec{x}}^{(0)}\right)(S) \\
& \equiv P_{\vec{x}, S}^{(w)}\left(P_{\vec{x}, S}^{(w-1)}\left(\cdots P_{\vec{x}, S}^{(1)}\left(M_{\vec{x}}^{(0)}(S)\right) \cdots\right)\right),
\end{aligned}
$$

where the projectors $P$ map functions to functions according to

$$
P_{\vec{x}, S}^{(r)}(h)=h+M_{\vec{x}}^{(r)}(S-S[h]) .
$$

The maps $M_{\vec{x}}^{(r)}(S)$ which occur on the right-hand sides of eqs. (B.4)-(B.5) are linear in the space of tensors (as in the case of a single variable) and are defined to act on elementary tensors $T$ by recursion in the number of variables,

$M_{\vec{x}}^{(r)}(T)= \begin{cases}M_{x_{1}}(T) & \text { for } r=0, \\ M_{x_{2}, \ldots, x_{n}}\left(T^{(1)} \otimes \cdots \otimes T^{(r)}\right) M_{x_{1}}\left(T^{(r+1)} \otimes \cdots \otimes T^{(w)}\right) & \text { for } r=1,2, \ldots, w-1, \\ M_{x_{2}, \ldots, x_{n}}(T) & \text { for } r=w .\end{cases}$ 
For the map $M_{\vec{x}}^{(r)}(T)$ to be non-vanishing, the last $w-r$ indices must depend on $x_{1}$. Its output is then given by a canonical function with argument $x_{1}$ and weight $w-r$, multiplied by an $x_{1}$-independent function of weight $r$. The projectors $P_{\vec{x}, S}^{(r)}(h)$ add such functions to their input, thus gradually constructing a function in canonical form, starting with functions of $x_{1}$ with weight $w$ down to weight 1 and repeating the process for the remaining variables $x_{2}, x_{3}, \ldots, x_{n}$. As a result, the multivariate map $M_{\vec{x}}(S)$ generates a function of the form

$$
\sum_{\left(i_{1}, \ldots, i_{n}\right)} c_{i_{1}, \ldots, i_{n}} G\left(\vec{a}_{i_{n}} ; x_{n}\right) \cdots G\left(\vec{a}_{i_{1}} ; x_{1}\right)
$$

where the $\vec{a}_{i_{k}}$ are independent of $x_{1}, \ldots, x_{k}$. This expression is by definition in canonical form. For a single variable, the indices are in fact constants and as slight abuse of terminology this is what we occasionally refer to as canonical form, bearing in mind that eq. (B.7) is the proper definition of canonical form in the multivariate case.

Having defined the multivariate map, let us point out the difference with respect to the procedure described in ref. [76]. A slight generalization resides in the definition of $M_{\vec{x}}^{(r)}(T)$. In particular, two of the maps on the right-hand side of eq. (B.6) depend on all remaining variables $x_{2}, \ldots, x_{n}$, rather than just the next variable $x_{2}$. This alteration allows us to reconstruct functions of more than two variables which have a sufficiently factorized form.

A simple example illustrates the point. Consider $g(x, y, z)=\log x \log y \log z$. This function is already in canonical form, but the algorithm should nonetheless be able to reconstruct this function from its symbol. In this case the weight is $w=3$, and we set $\vec{x}=\{x, y, z\}$. The symbol of $g(x, y, z)$ is given by

$$
S=x \otimes y \otimes z+x \otimes z \otimes y+y \otimes x \otimes z+y \otimes z \otimes x+z \otimes x \otimes y+z \otimes y \otimes x .
$$

Let us apply the map to this symbol,

$$
M_{\vec{x}}(S)=\left(P_{\vec{x}, S}^{(3)} \circ P_{\vec{x}, S}^{(2)} \circ P_{\vec{x}, S}^{(1)} \circ M_{\vec{x}}^{(0)}\right)(S) .
$$

Since $S$ contains no tensors with all three entries depending on $x$, the first map gives zero,

$$
M_{\vec{x}}^{(0)}(S)=M_{x}(S)=0 .
$$

Subsequently, the first projector acts on this result. Inserting its definition from eq. (B.5), it reduces to applying the map $M_{\vec{x}}^{(1)}(S)$, which is non-zero only for tensors whose last two entries depend on $x$. Since $S$ does not contain such tensors, the result is zero,

$$
P_{\vec{x}}^{(1)}(0)=M_{\vec{x}}^{(1)}(S)=0 \text {. }
$$

A non-vanishing contribution is found in the next step, coming from the two tensors which contain $x$ in the last entry.

$$
P_{\vec{x}}^{(2)}(0)=M_{\vec{x}}^{(2)}(S)=M_{y, z}(y \otimes z) M_{x}(x)+M_{y, z}(z \otimes y) M_{x}(x) .
$$

From the definition in eq. (B.3) we have $M_{x}(x)=G(0 ; x)=\log x$. The other map $M_{y, z}$ is computed along the very same lines, with the results $M_{y, z}(y \otimes z)=0$ and $M_{y, z}(z \otimes y)=$ 
$\log y \log z$. By now we have that $M_{\vec{x}}(S)=P_{\vec{x}, S}^{(3)}(h)$, with $h=P_{\vec{x}}^{(2)}(0)=\log x \log y \log z$. Because $S=S[h]$, the last projector $P_{\vec{x}}^{(3)}$ becomes the identity, resulting in

$$
M_{\vec{x}}(S)=\log x \log y \log z=g(x, y, z),
$$

as required, because our algorithm should reconstruct the original function from its symbol. This example shows that it is crucial to have both variables $y$ and $z$ as parameters in the maps $M_{y, z}$ in eq. (B.12). If one would use a single variable only, then the vanishing maps $M_{y}(z \otimes y)=M_{y}(y \otimes z)=0$, as one can easily verify from eq. (B.3), would lead to a vanishing, and hence incorrect, result. This illustrates the purpose of the slight generalization of the map.

In conclusion, the second step of the algorithm constructs from the symbol $S[g(\vec{x})]$ an expression in canonical form, which has the same symbol as $g(\vec{x})$, by applying the map $M_{\vec{x}}(S)$ in eq. (B.4).

The resulting expression differs from the original function $g(\vec{x})$ only by terms proportional to transcendental constants, because such terms are in the kernel of the symbol map. Finding these missing terms is the task of the next step in the algorithm.

The third step in the algorithm revolves around the coproduct of multiple polylogarithms, which generalizes the concept of the symbol. Before describing how it may be used to construct missing terms proportional to transcendental constants, let us first define the required coproducts of the form $\Delta_{p, q, \ldots, r}$. They are derived from the general coproduct $\Delta$, which is defined by its action on iterated integrals with a freely specified base point of integration, see eq. (A.16), cf. ref. [71]

$$
\begin{aligned}
& \Delta\left[I\left(a_{0} ; a_{1}, \ldots, a_{n} ; a_{n+1}\right)\right]= \\
& \sum_{k=0}^{n} \sum_{0=i_{0}<i_{1}<\cdots<i_{k}<i_{k+1}=n+1} I\left(a_{0} ; a_{i_{1}}, \ldots, a_{i_{k}} ; a_{n+1}\right) \otimes \prod_{p=0}^{k} I\left(a_{i_{p}} ; a_{i_{p}+1}, \ldots, a_{i_{p+1}-1} ; a_{i_{p+1}}\right) .
\end{aligned}
$$

The right-hand side of eq. (B.14) consists of tensors with two entries, each entry having a weight between 0 and $n$, such that the two weights add up to $n$, the weight of the original function. ${ }^{13}$ All possible pairs of weights are thus $(0, n),(1, n-1), \ldots,(n, 0)$. Grouping the tensors by those pairs of weights decomposes the coproduct into

$$
\Delta=\sum_{p+q=n} \Delta_{p, q}
$$

In other words, the action of $\Delta_{p, q}$ on $I\left(a_{0} ; a_{1}, \ldots, a_{n} ; a_{n+1}\right)$ yields the subset of terms in eq. (B.14) of tensors with weight $(p, q)$. Besides the operator $\Delta_{p, q}$, the third step of our algorithm also uses operators with multiple indices $\Delta_{p, q, \ldots, r}$. Those are defined recursively in terms of $\Delta_{p, q}$. For example, $\Delta_{p, q, r}$ is defined by application of $\Delta_{q, r}$ to the second entry

\footnotetext{
${ }^{13}$ The weight of $I\left(a_{0} ; a_{1}, \ldots, a_{n} ; a_{n+1}\right)$ is equal to its number of indices, $n$. Likewise, a pair of weights $(p, q)$ is attributed to a tensor $T_{p} \otimes T_{q}$ where the weights of $T_{p}$ and $T_{q}$ are $p$ and $q$, respectively.
} 
of all tensors obtained from $\Delta_{p, q+r}$. These are the definitions of the coproducts which are needed in the third step of the algorithm. For more details, see ref. [75].

The coproducts thus defined may be employed to construct missing terms proportional to transcendental constants, starting with the lowest-weight constants, cf. ref. [76]. To be specific, in the previous step of the algorithm we constructed a function $h(\vec{x})$ such that $S[g(\vec{x})-h(\vec{x})]=0$. This means that the difference $g(\vec{x})-h(\vec{x})$ must be proportional to transcendental constants. Following the recipe in the third step of the algorithm we act on this difference with the coproduct $\Delta_{2,1, \ldots, 1}$ to first find terms proportional to $\zeta_{2}$. The coproduct takes the form

$$
\Delta_{2,1, \ldots, 1}[g(\vec{x})-h(\vec{x})]=\sum_{\left(i_{3}, \ldots, i_{w}\right)} L_{i_{3}, \ldots, i_{w}}(\vec{x}) \otimes \log R_{i_{3}}(\vec{x}) \otimes \cdots \otimes \log R_{i_{w}}(\vec{x}),
$$

where $L_{i_{3}, \ldots, i_{w}}$ is a linear combination of weight-two multiple polylogarithms, and the $R_{i_{3}}, \ldots, R_{i_{w}}$ are rational functions. Since the weight-two object $L_{i_{3}, \ldots, i_{w}}$ must be proportional to $\zeta_{2}$, we write $L_{i_{3}, \ldots, i_{w}}=k \zeta_{2}$ for some rational number $k$. This constant of proportionality can be determined by numerical evaluation at some specific values for $\vec{x}$ using Ginac [85] and running the PSLQ algorithm [86, 87]. The hereby obtained transcendental constant $k \zeta_{2}$ multiplies a polylogarithmic function, whose symbol is given by $R_{i_{3}} \otimes \cdots \otimes R_{i_{w}}$, arising from the tail of the arguments of eq. (B.16). Feeding this symbol back into the first step of this algorithm and collecting the resulting multiple polylogarithms from the output of step two produces a function $h_{2}(\vec{x})$ in canonical form, which is to multiply the constant $k \zeta_{2}$. As a consequence we have that

$$
\Delta_{2,1, \ldots, 1}\left[g(\vec{x})-h(\vec{x})-k \zeta_{2} h_{2}(\vec{x})\right]=0,
$$

and we conclude that the difference $g(\vec{x})-h(\vec{x})-k \zeta_{2} h_{2}(\vec{x})$ is equal to terms proportional to transcendental constants of weight three and higher, which are in the kernel of $\Delta_{2,1, \ldots, 1}$. Iterating this procedure with the coproducts $\Delta_{3,1, \ldots, 1}, \ldots, \Delta_{w-1,1}$ allows us to moreover reconstruct the other missing constants with transcendental weight $3, \ldots, w-1$, respectively. The final output of the third step in the algorithm is thus a rewritten version of the original function in canonical form.

Let us conclude this appendix by demonstrating an explicit application of the algorithm, involving in particular the use of the coproduct. To this end, we consider the expression in the first line of eq. (4.19), $\widetilde{\mathcal{F}}^{(2)}=2 R(\chi)^{2} g(\chi)$, where the interesting part is given by the weight-three function

$$
g(\chi)=\frac{1}{4}\left(G\left(\rho_{1}, 0, \rho_{1} ; 1\right)-G\left(\rho_{1}, 0, \rho_{2} ; 1\right)-G\left(\rho_{2}, 0, \rho_{1} ; 1\right)+G\left(\rho_{2}, 0, \rho_{2} ; 1\right)\right) .
$$

It depends on a single variable $\chi$ through its indices, $\rho_{1}=\frac{\chi}{\chi-1}$ and $\rho_{2}=\frac{1}{1-\chi}$. We wish to express this in terms of multiple polylogarithms with constant indices and argument $\chi$. Following the algorithm, we start by computing the symbol of $g(\chi)$,

$$
S[g(\chi)]=\chi \otimes(1-\chi) \otimes \chi-\chi \otimes \chi \otimes \chi+\chi \otimes(1+\chi) \otimes \chi .
$$


Application of the map from step two yields a multiple polylogarithm in canonical form with the same symbol,

$$
h(\chi)=M_{\chi}(S[g(\chi)])=G(0,-1,0 ; \chi)-G(0,0,0 ; \chi)+G(0,1,0 ; \chi) .
$$

Indeed, the symbol of the difference vanishes, $S(g(\chi)-h(\chi))=0$. Yet the functions $g(\chi)$ and $h(\chi)$ are not equal, because they differ by terms proportional to zeta values up to weight three, in this case $\zeta_{2}$ and $\zeta_{3}$. According to the third step of the algorithm, the missing terms proportional to $\zeta_{2}$ are obtained first by acting with the coproduct $\Delta_{2,1}$ on the difference,

$$
\Delta_{2,1}[g(\chi)-h(\chi)]=c_{2} \otimes G(0 ; \chi)=\Delta_{2,1}\left[c_{2} G(0 ; \chi)\right]
$$

where we expect that $c_{2}=k \zeta_{2}$ for some rational number $k$. Explicitly, we find

$$
\begin{aligned}
c_{2}= & -\frac{1}{4} G\left(\frac{\chi}{\chi-1}, \frac{1}{1-\chi} ; 1\right)+\frac{1}{4} G\left(\frac{1}{1-\chi}, \frac{\chi}{\chi-1} ; 1\right)-\operatorname{Li}_{2}(\chi)-\operatorname{Li}_{2}(-\chi)+\frac{1}{2} \operatorname{Li}_{2}\left(\frac{\chi-1}{\chi}\right) \\
& -\frac{1}{2} \operatorname{Li}_{2}(1-\chi)-\log (\chi) \log (1+\chi)+\frac{1}{2} \log ^{2}(\chi)-\log (1-\chi) \log (\chi) .
\end{aligned}
$$

Evaluating this expression at any value of $\chi$ with Ginac yields

$$
c_{2}=-0.822467033424113218 \ldots=-\frac{1}{2} \zeta_{2} .
$$

Inserting this result for $c_{2}$ into eq. (B.21), we conclude that $\Delta_{2,1}\left[g(\chi)-\left(h(\chi)-\frac{1}{2} \zeta_{2} G(0 ; \chi)\right)\right]$ $=0$. To find the last missing contribution proportional to $\zeta_{3}$, it suffices to evaluate the remaining difference numerically at a single point,

$$
g(\chi)-\left(h(\chi)-\frac{1}{2} \zeta_{2} G(0 ; \chi)\right)=-0.601028451579797142 \ldots=-\frac{1}{2} \zeta_{3} .
$$

We finally conclude that

$$
\begin{aligned}
g(\chi) & =h(\chi)-\frac{1}{2} \zeta_{2} G(0 ; \chi)-\frac{1}{2} \zeta_{3} \\
& =G(0,-1,0 ; \chi)-G(0,0,0 ; \chi)+G(0,1,0 ; \chi)-\frac{1}{2} \zeta_{2} G(0 ; \chi)-\frac{1}{2} \zeta_{3} .
\end{aligned}
$$

We have thus succeeded in expressing $g(\chi)$ in terms of multiple polylogarithms with constant indices and argument $\chi$. Inserting this result into $\widetilde{\mathcal{F}}^{(2)}=2 R(\chi)^{2} g(\chi)$ reproduces the second line of eq. (4.19). It is now a simple matter of applying eq. (A.5) for multiple polylogarithms with constant indices, to rewrite $g(\chi)$ in terms of classical polylogarithms, thus reducing the expression to the form given in the last line of eq. (4.19).

This completes the illustration of our algorithm in a practical example and thereby also completes our description of each of the three steps in the algorithm to rewrite multiple polylogarithms in canonical form.

Open Access. This article is distributed under the terms of the Creative Commons Attribution License (CC-BY 4.0), which permits any use, distribution and reproduction in any medium, provided the original author(s) and source are credited. 


\section{References}

[1] E. Laenen, K.J. Larsen and R. Rietkerk, Imaginary parts and discontinuities of Wilson line correlators, Phys. Rev. Lett. 114 (2015) 181602 [arXiv:1410.5681] [INSPIRE].

[2] D.R. Yennie, S.C. Frautschi and H. Suura, The infrared divergence phenomena and high-energy processes, Annals Phys. 13 (1961) 379 [INSPIRE].

[3] G.F. Sterman, Infrared divergences in perturbative QCD, AIP Conf. Proc. 74 (1981) 22 [INSPIRE].

[4] J.G.M. Gatheral, Exponentiation of eikonal cross sections in nonabelian gauge theories, Phys. Lett. B 133 (1983) 90 [INSPIRE].

[5] J. Frenkel and J.C. Taylor, Non-abelian eikonal exponentiation, Nucl. Phys. B 246 (1984) 231 [inSPIRE].

[6] L. Magnea and G.F. Sterman, Analytic continuation of the Sudakov form-factor in QCD, Phys. Rev. D 42 (1990) 4222 [INSPIRE].

[7] L. Magnea, Analytic resummation for the quark form-factor in QCD, Nucl. Phys. B 593 (2001) 269 [hep-ph/0006255] [INSPIRE].

[8] E. Gardi, E. Laenen, G. Stavenga and C.D. White, Webs in multiparton scattering using the replica trick, JHEP 11 (2010) 155 [arXiv: 1008.0098] [INSPIRE].

[9] A. Mitov, G. Sterman and I. Sung, Diagrammatic exponentiation for products of Wilson lines, Phys. Rev. D 82 (2010) 096010 [arXiv:1008.0099] [InSPIRE].

[10] E. Gardi and C.D. White, General properties of multiparton webs: proofs from combinatorics, JHEP 03 (2011) 079 [arXiv: 1102.0756] [INSPIRE].

[11] E. Gardi, J.M. Smillie and C.D. White, On the renormalization of multiparton webs, JHEP 09 (2011) 114 [arXiv: 1108.1357] [INSPIRE].

[12] M. Dukes, E. Gardi, E. Steingrimsson and C.D. White, Web worlds, web-colouring matrices and web-mixing matrices, J. Comb. Theory Ser. A 120 (2013) 1012 [arXiv:1301.6576] [INSPIRE].

[13] M. Dukes, E. Gardi, H. McAslan, D.J. Scott and C.D. White, Webs and posets, JHEP 01 (2014) 024 [arXiv:1310.3127] [INSPIRE].

[14] E. Gardi, J.M. Smillie and C.D. White, The non-Abelian exponentiation theorem for multiple Wilson lines, JHEP 06 (2013) 088 [arXiv: 1304.7040] [INSPIRE].

[15] A.M. Polyakov, Gauge fields as rings of glue, Nucl. Phys. B 164 (1980) 171 [InSPIRE].

[16] I.Y. Aref'eva, Quantum contour field equations, Phys. Lett. B 93 (1980) 347 [INSPIRE].

[17] V.S. Dotsenko and S.N. Vergeles, Renormalizability of phase factors in the non-abelian gauge theory, Nucl. Phys. B 169 (1980) 527 [INSPIRE].

[18] R.A. Brandt, F. Neri and M.-a. Sato, Renormalization of loop functions for all loops, Phys. Rev. D 24 (1981) 879 [inSPIRE].

[19] G.P. Korchemsky and A.V. Radyushkin, Loop space formalism and renormalization group for the infrared asymptotics of QCD, Phys. Lett. B 171 (1986) 459 [INSPIRE].

[20] G.P. Korchemsky and A.V. Radyushkin, Infrared asymptotics of perturbative QCD: renormalization properties of the Wilson loops in higher orders of perturbation theory, Sov. J. Nucl. Phys. 44 (1986) 877 [inSPIRE]. 
[21] G.P. Korchemsky and A.V. Radyushkin, Renormalization of the Wilson loops beyond the leading order, Nucl. Phys. B 283 (1987) 342 [INSPIRE].

[22] N. Kidonakis, G. Oderda and G.F. Sterman, Evolution of color exchange in QCD hard scattering, Nucl. Phys. B 531 (1998) 365 [hep-ph/9803241] [INSPIRE].

[23] N. Kidonakis and G.F. Sterman, Resummation for QCD hard scattering, Nucl. Phys. B 505 (1997) 321 [hep-ph/9705234] [inSPIRE].

[24] T. Becher and M. Neubert, Infrared singularities of scattering amplitudes in perturbative QCD, Phys. Rev. Lett. 102 (2009) 162001 [arXiv:0901.0722] [INSPIRE].

[25] E. Gardi and L. Magnea, Factorization constraints for soft anomalous dimensions in QCD scattering amplitudes, JHEP 03 (2009) 079 [arXiv:0901.1091] [INSPIRE].

[26] N. Kidonakis, Two-loop soft anomalous dimensions and NNLL resummation for heavy quark production, Phys. Rev. Lett. 102 (2009) 232003 [arXiv:0903.2561] [inSPIRE].

[27] A. Grozin, J.M. Henn, G.P. Korchemsky and P. Marquard, Three loop cusp anomalous dimension in QCD, Phys. Rev. Lett. 114 (2015) 062006 [arXiv: 1409.0023] [INSPIRE].

[28] D. Correa, J. Henn, J. Maldacena and A. Sever, The cusp anomalous dimension at three loops and beyond, JHEP 05 (2012) 098 [arXiv:1203.1019] [INSPIRE].

[29] J.M. Henn and T. Huber, Systematics of the cusp anomalous dimension, JHEP 11 (2012) 058 [arXiv:1207.2161] [INSPIRE].

[30] J.M. Henn and T. Huber, The four-loop cusp anomalous dimension in $\mathcal{N}=4$ super Yang-Mills and analytic integration techniques for Wilson line integrals, JHEP 09 (2013) 147 [arXiv: 1304.6418] [INSPIRE].

[31] S.M. Aybat, L.J. Dixon and G.F. Sterman, The two-loop anomalous dimension matrix for soft gluon exchange, Phys. Rev. Lett. 97 (2006) 072001 [hep-ph/0606254] [INSPIRE].

[32] S.M. Aybat, L.J. Dixon and G.F. Sterman, The two-loop soft anomalous dimension matrix and resummation at next-to-next-to leading pole, Phys. Rev. D 74 (2006) 074004 [hep-ph/0607309] [INSPIRE].

[33] T. Becher and M. Neubert, Infrared singularities of QCD amplitudes with massive partons, Phys. Rev. D 79 (2009) 125004 [arXiv:0904.1021] [InSPIRE].

[34] A. Ferroglia, M. Neubert, B.D. Pecjak and L.L. Yang, Two-loop divergences of scattering amplitudes with massive partons, Phys. Rev. Lett. 103 (2009) 201601 [arXiv:0907.4791] [INSPIRE].

[35] A. Ferroglia, M. Neubert, B.D. Pecjak and L.L. Yang, Two-loop divergences of massive scattering amplitudes in non-abelian gauge theories, JHEP 11 (2009) 062 [arXiv:0908.3676] [INSPIRE].

[36] A. Mitov, G.F. Sterman and I. Sung, Computation of the soft anomalous dimension matrix in coordinate space, Phys. Rev. D 82 (2010) 034020 [arXiv:1005.4646] [INSPIRE].

[37] N. Kidonakis, Next-to-next-to-leading soft-gluon corrections for the top quark cross section and transverse momentum distribution, Phys. Rev. D 82 (2010) 114030 [arXiv:1009.4935] [INSPIRE].

[38] E. Gardi, From webs to polylogarithms, JHEP 04 (2014) 044 [arXiv:1310.5268] [INSPIRE].

[39] G. Falcioni, E. Gardi, M. Harley, L. Magnea and C.D. White, Multiple gluon exchange webs, JHEP 10 (2014) 010 [arXiv: 1407.3477] [InSPIRE]. 
[40] S. Catani, D. de Florian and G. Rodrigo, Space-like (versus time-like) collinear limits in QCD: is factorization violated?, JHEP 07 (2012) 026 [arXiv:1112.4405] [INSPIRE].

[41] J.R. Forshaw, M.H. Seymour and A. Siodmok, On the breaking of collinear factorization in QCD, JHEP 11 (2012) 066 [arXiv:1206.6363] [INSPIRE].

[42] S. Catani and M. Ciafaloni, Generalized coherent state for soft gluon emission, Nucl. Phys. B 249 (1985) 301 [inSPIRE].

[43] S. Catani, M. Ciafaloni and G. Marchesini, Non-cancelling infrared divergences in QCD coherent state, Nucl. Phys. B 264 (1986) 588 [inSPIRE].

[44] J.R. Forshaw, A. Kyrieleis and M.H. Seymour, Super-leading logarithms in non-global observables in QCD, JHEP 08 (2006) 059 [hep-ph/0604094] [INSPIRE].

[45] J.R. Forshaw, A. Kyrieleis and M.H. Seymour, Super-leading logarithms in non-global observables in QCD: colour basis independent calculation, JHEP 09 (2008) 128 [arXiv:0808.1269] [INSPIRE].

[46] G.P. Korchemsky and G. Marchesini, Structure function for large $x$ and renormalization of Wilson loop, Nucl. Phys. B 406 (1993) 225 [hep-ph/9210281] [INSPIRE].

[47] E. Gardi, On the quark distribution in an on-shell heavy quark and its all-order relations with the perturbative fragmentation function, JHEP 02 (2005) 053 [hep-ph/0501257] [INSPIRE].

[48] Z. Bern, L.J. Dixon, D.C. Dunbar and D.A. Kosower, One-loop n-point gauge theory amplitudes, unitarity and collinear limits, Nucl. Phys. B 425 (1994) 217 [hep-ph/9403226] [INSPIRE].

[49] Z. Bern, L.J. Dixon, D.C. Dunbar and D.A. Kosower, Fusing gauge theory tree amplitudes into loop amplitudes, Nucl. Phys. B 435 (1995) 59 [hep-ph/9409265] [INSPIRE].

[50] Z. Bern, L.J. Dixon and D.A. Kosower, Progress in one loop QCD computations, Ann. Rev. Nucl. Part. Sci. 46 (1996) 109 [hep-ph/9602280] [INSPIRE].

[51] R. Britto, F. Cachazo and B. Feng, Generalized unitarity and one-loop amplitudes in $N=4$ super-Yang-Mills, Nucl. Phys. B 725 (2005) 275 [hep-th/0412103] [INSPIRE].

[52] R. Britto, E. Buchbinder, F. Cachazo and B. Feng, One-loop amplitudes of gluons in SQCD, Phys. Rev. D 72 (2005) 065012 [hep-ph/0503132] [INSPIRE].

[53] C. Anastasiou, R. Britto, B. Feng, Z. Kunszt and P. Mastrolia, D-dimensional unitarity cut method, Phys. Lett. B 645 (2007) 213 [hep-ph/0609191] [INSPIRE].

[54] D. Forde, Direct extraction of one-loop integral coefficients, Phys. Rev. D 75 (2007) 125019 [arXiv: 0704 .1835] [INSPIRE].

[55] P. Mastrolia, Double-cut of scattering amplitudes and Stokes' theorem, Phys. Lett. B 678 (2009) 246 [arXiv:0905.2909] [INSPIRE].

[56] D.A. Kosower and K.J. Larsen, Maximal unitarity at two loops, Phys. Rev. D 85 (2012) 045017 [arXiv:1108.1180] [INSPIRE].

[57] S. Caron-Huot and K.J. Larsen, Uniqueness of two-loop master contours, JHEP 10 (2012) 026 [arXiv: 1205.0801] [InSPIRE].

[58] T. Gehrmann and E. Remiddi, Differential equations for two loop four point functions, Nucl. Phys. B 580 (2000) 485 [hep-ph/9912329] [INSPIRE]. 
[59] J.M. Henn, Multiloop integrals in dimensional regularization made simple, Phys. Rev. Lett. 110 (2013) 251601 [arXiv:1304.1806] [INSPIRE].

[60] G. 't Hooft and M.J.G. Veltman, Diagrammar, CERN, Geneva Switzerland (1973) [InSPIRE].

[61] O. Erdoğan and G. Sterman, Gauge theory webs and surfaces, Phys. Rev. D 91 (2015) 016003 [arXiv:1112.4564] [InSPIRE].

[62] O. Erdoğan, Coordinate-space singularities of massless gauge theories, Phys. Rev. D 89 (2014) 085016 [arXiv:1312.0058] [InSPIRE].

[63] O. Erdoğan and G. Sterman, Ultraviolet divergences and factorization for coordinate-space amplitudes, Phys. Rev. D 91 (2015) 065033 [arXiv:1411.4588] [InSPIRE].

[64] O.T. Engelund, Lagrangian insertion in the light-like limit and the super-correlators/super-amplitudes duality, arXiv:1502.01934 [INSPIRE].

[65] G. Sterman, An introduction to quantum field theory, Cambridge University Press, Cambridge U.K. (1993) [INSPIRE].

[66] Y.-T. Chien, M.D. Schwartz, D. Simmons-Duffin and I.W. Stewart, Jet physics from static charges in AdS, Phys. Rev. D 85 (2012) 045010 [arXiv:1109.6010] [InSPIRE].

[67] D. Correa, J. Maldacena and A. Sever, The quark anti-quark potential and the cusp anomalous dimension from a TBA equation, JHEP 08 (2012) 134 [arXiv:1203.1913] [INSPIRE].

[68] M. Beneke and V.M. Braun, Power corrections and renormalons in Drell-Yan production, Nucl. Phys. B 454 (1995) 253 [hep-ph/9506452] [INSPIRE].

[69] Y. Schröder, The static potential in QCD to two loops, Phys. Lett. B 447 (1999) 321 [hep-ph/9812205] [INSPIRE].

[70] J.C. Collins, D.E. Soper and G.F. Sterman, All order factorization for Drell-Yan cross-sections, Phys. Lett. B 134 (1984) 263 [INSPIRE].

[71] A.B. Goncharov, Galois symmetries of fundamental groupoids and noncommutative geometry, Duke Math. J. 128 (2005) 209 [math. AG/0208144] [INSPIRE].

[72] A.B. Goncharov, M. Spradlin, C. Vergu and A. Volovich, Classical polylogarithms for amplitudes and Wilson loops, Phys. Rev. Lett. 105 (2010) 151605 [arXiv:1006.5703] [INSPIRE].

[73] F. Brown, On the decomposition of motivic multiple zeta values, arXiv:1102.1310 [INSPIRE].

[74] C. Duhr, H. Gangl and J.R. Rhodes, From polygons and symbols to polylogarithmic functions, JHEP 10 (2012) 075 [arXiv: 1110.0458] [INSPIRE].

[75] C. Duhr, Hopf algebras, coproducts and symbols: an application to Higgs boson amplitudes, JHEP 08 (2012) 043 [arXiv: 1203.0454] [INSPIRE].

[76] C. Anastasiou, C. Duhr, F. Dulat and B. Mistlberger, Soft triple-real radiation for Higgs production at N3LO, JHEP 07 (2013) 003 [arXiv: 1302.4379] [INSPIRE].

[77] D. Maître, HPL, a Mathematica implementation of the harmonic polylogarithms, Comput. Phys. Commun. 174 (2006) 222 [hep-ph/0507152] [INSPIRE].

[78] D. Maître, Extension of HPL to complex arguments, Comput. Phys. Commun. 183 (2012) 846 [hep-ph/0703052] [INSPIRE]. 
[79] E. Laenen, G. Stavenga and C.D. White, Path integral approach to eikonal and next-to-eikonal exponentiation, JHEP 03 (2009) 054 [arXiv: 0811.2067] [INSPIRE].

[80] A. Mitov, G.F. Sterman and I. Sung, The massive soft anomalous dimension matrix at two loops, Phys. Rev. D 79 (2009) 094015 [arXiv:0903.3241] [InSPIRE].

[81] M. Galassi et al., GNU Scientific Library - reference manual, 3rd ed., Network Theory Ltd (2009).

[82] E. Remiddi and J.A.M. Vermaseren, Harmonic polylogarithms, Int. J. Mod. Phys. A 15 (2000) 725 [hep-ph/9905237] [INSPIRE].

[83] J. Vollinga and S. Weinzierl, Numerical evaluation of multiple polylogarithms, Comput. Phys. Commun. 167 (2005) 177 [hep-ph/0410259] [INSPIRE].

[84] A.B. Goncharov, A simple construction of Grassmannian polylogarithms, arXiv:0908.2238 [INSPIRE].

[85] C.W. Bauer, A. Frink and R. Kreckel, Introduction to the GiNaC framework for symbolic computation within the $C++$ programming language, cs/0004015.

[86] H.R.P. Ferguson and D.H. Bailey, A polynomial time, numerically stable integer relation algorithm, RNR Tech. Rept. RNR-91-032 (1992).

[87] H.R.P. Ferguson, D.H. Bailey and S. Arno, Analysis of PSLQ, an integer relation finding algorithm, Math. Comput. 68 (1999) 351. 